The National Oceanographic Data Center is sponsored by six government agencles having an interest in the marine environment; it is governed by an Advisory Board composed of representatives of these activities and the National Academy of Sciences. The U. S. Navy Hydrographic Office is assigned responsibility for management of the National Oceanographic Data Center.

The sponsoring agencies are:

ATOMIC ENERGY COMMISSION

BUREAU OF COMMERCIAL FISHERIES

COAST AND GEODETIC SURVEY

DEPARTMEIVT OF THE NAVY

NATIONAT SCIENCE FOUNDATION

WEATHER BUREAU

Printed by

U. S. NAVY HYDROGRAPHIC OFFICE

WASHINGTON 25, D. C.

1962

Price 90 conts 


\title{
NATIONAL OCEANOGRAPHIC DATA CENTER
}

\author{
MANUAL SERIES
}

PROCESSING PHYSICAL AND CHEMICAL DATA FROM OCEANOGRAPHIC STATIONS

PUBLICATION $M-2$

1962

DATA IIBRARY 


$$
\text { (2) }
$$

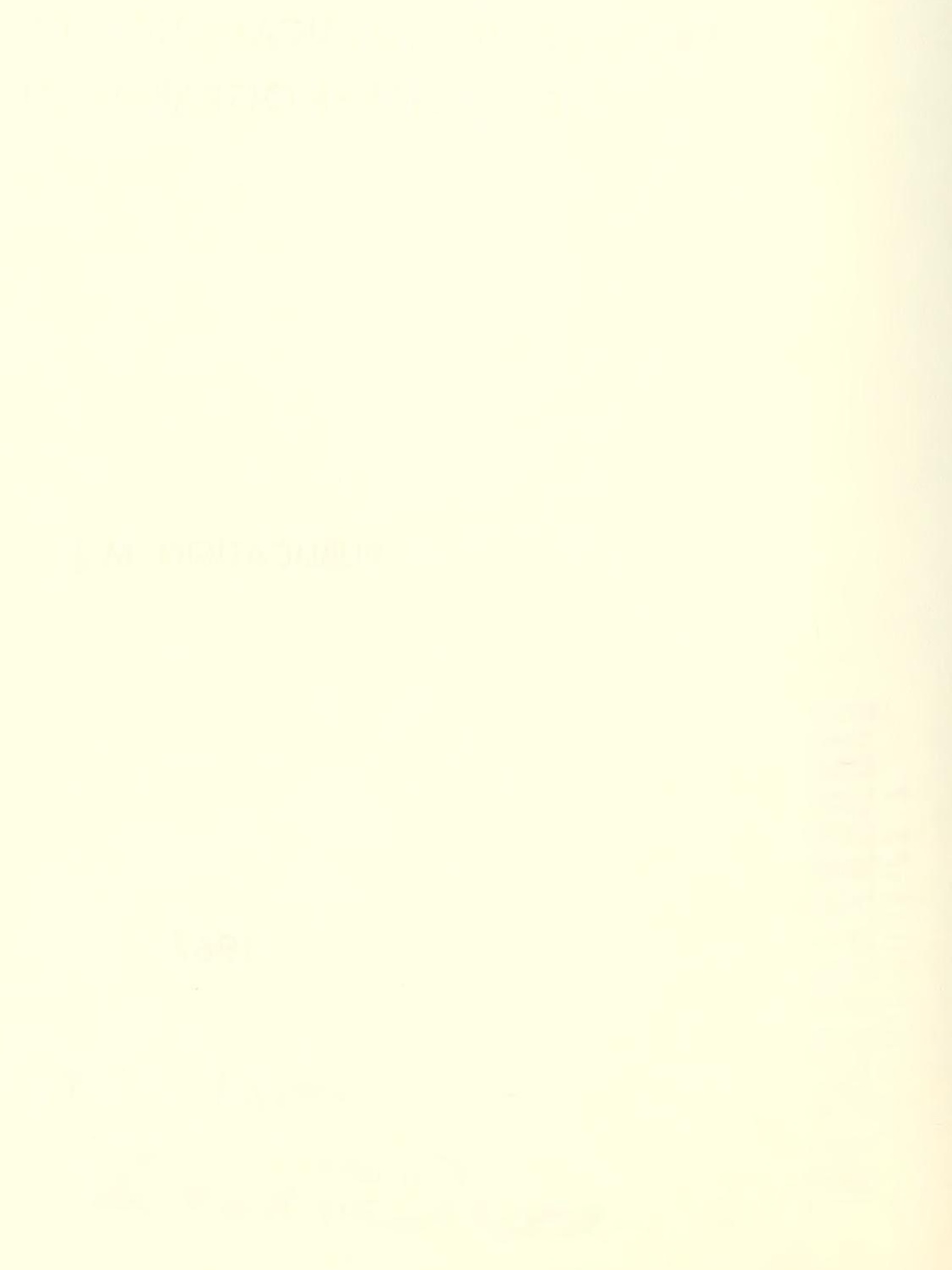


This publication describes the methods used at the National Oceanographic Data Center (NODC) for reducing processed physical and chemical oceanographic station data to a standard format. It is intended also for use by other agencies or contributors interested in furnishing oceanographic data to NODC or making their data systems compatible with those of NODC. An outline of the format of the Computed Card is included in order to acquaint the users of the NODC oceanographic station data holdings with the end product of the routinely performed computations.

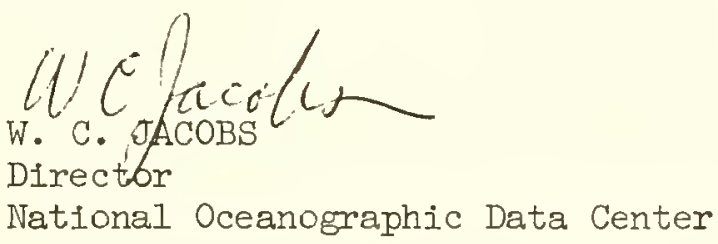





\section{Page}

FOREWORD

LIST OF TABLES

Vii

INIRODUCTION

GEINERAL

CODING THE MASTER CARD INFORMATION

ENIRIES FOR SURFACE ENVIRONMENTAL INFORMATION

(MASTER CARD)

$\begin{array}{ll}\text { CODING THE DETAII CARD INFORMATION } & 10\end{array}$

ENTRIES FOR SUBSURFACE OBSERVATIONS (DETAII CARD) II

INSTRUCTIONS FOR PUNCHING NODC OCEANOGRAPHIC

STATION CARDS FROM THE PHYSICAL AND CHFMICAL

DATA CODING FORM

MASTER CARD

DETAIL CARD

THE COMPUIED DATA CARD

COMPUTED MASTER CARD

COMPUTED OBSERVED CARD

COMPUTED STANDARD DEPTH CARD

CARD TYPE IDENTIFICATION CODE

APPENDIX I - PHYSICAL AND CHEMICAL DATA FORM, NHO-NODC-3167/I (9-6I) AND OCEANOGRAPHIC 

TABIE

NUMBER

1. COUNIRY CODE - International Geophysical Year

$$
\text { (IGY) Code }
$$

2. TENTH CONVERSION - Conversion forom Seconds (of Position or Minutes (of Time) to Tenths of Minutes or Hour

3. MARSDEN SQUARE CHART

4. TIME - Conversion from Local to Greenwich Mean Time (GMT)

5. DEPTH - Conversion from Fathoms to Meters 30

6. DEPTH - Conversion from Feet to Meters (tenths) 32

7. ADDITIONAI OBSERVATIONS 34

8. WATER COLOR - FOrel-Ule Scale and Conversion from other Color Scales 35

9. DIRECTION - In Tens of Degrees from which Waves and/or winds are Coming

10. DIRECTION - Conversion from Points, Quarter Points, or a Scale of 32 , To a Scale of 36 Points 37

11. HEIGHT - WMO Code 1555 for Recording Height of the Dominant Waves

12. PERIOD - WMO Code 3155 for Recording Period of the Dominant Waves

13. SEA STATE - Conversion from the Douglas Scale to WMO Code 3700

14. WIND SPEED - Conversion from Meters per Second to Knots

15. WIND SPEED - Conversion from Miles per Hour to Knots 42

16. WIND SPEED - Conversion from Kilometers per Hour to Knots 
17. WIND SPEED - Conversion from Feet per Second to Knots 44

18. WIND FORCE - Conversion frrom Knots, Meters per Second, Kilometers per Hour, and Miles per Hour to the Beaufort Wind Scale

19. ATMOSPHERIC PRESSURE - Conversion from Inches of Mercury to Millibars

20. ATMOSPHERIC PRESSURE - Conversion from Millimeters of Mercury to Millibars

21. TEMPERATURE - Conversion from Fahrenheit to Centigrade

22. PRESENT WEATHER - WMO Code 4501 for Recording Present Weather

58

23. PRESENT WEATHFR - Conversion from Beaufort Weather Notation to WiMO Code 4501

24. PRESEIP WEATHER - Conversion from 1936 International Meteorological Organization Code to the WMO Code 4501

25. PRESENT WEATHER - WMO Code 4677 for Recording Present Weather

26. CLOUD TYPE (GENUS) - WMO Code 0500 fior Recording Cloud Type (Genus)

27. CLOUD AMOUNI - WMO Code 2700 for Recording Cloud Amount

28. VISIBILITY - WMO Code 4300 for Recording Visibility at Surface

29. PRECISION OF MEASUREMENT

30. SAIINITY - Conversion from Chlorinity to Salinity $(\%)$ 
TABLE

NUMBER

32. OXYGEN - Conversion from Mill1gram-Atoms per liter to Milliliters per Liter

33. PHOSPHORUS - Conversion from Micrograms per Liter of Inorganic $\mathrm{P}$ to Microgram-Atoms per liter of $P$

34. PHOSPHAIE - Conversion from Micrograms per Liter of $\mathrm{PO}_{4}$ to Microgram-Atoms per Liter of $\mathrm{PO}_{4}-\mathrm{P}$

35. PHOSPHORUS PENTOXIDE - COnversion from Micrograms per Liter of $\mathrm{P}_{2} \mathrm{O}_{5}$ to MicrogramAtoms per Iiter of $\mathrm{P}$

36. NITRITE - Conversion from Micrograms per Liter of $\mathrm{NO}_{2}$ to Microgram-Atoms per Liter of $\mathrm{NO}_{2}-\mathrm{N}$

37. NITRATE - Conversion from Micrograms per Liter of $\mathrm{NO}_{3}$ to Microgram-Atoms per Liter of $\mathrm{NO}_{3}-\mathrm{N}$

38. SIIICON - Conversion from Micrograms per Liter of $S 1$ to Microgram-Atoms per Iiter of $\mathrm{Si}$

39. SILICON DIOXIDE - Conversion from Micrograms per Iiter of $\mathrm{SiO}_{2}$ to Microgram-Atoms per Liter of $\mathrm{SiO}_{2}-\mathrm{Si}$

40. SIIICATE - Conversion from Milligrams per Liter of $\mathrm{SIO}_{3}$ to Microgram-Atoms per Liter of $\mathrm{SiO}_{3}-\mathrm{SI}$ 



\section{INTRODUCTION}

This manual is intended to provide the necessary instructions and conversion tables for reducing processed marine physical and chemical environmental data collected at oceanographic stations to a standard format. Instructions for punching the National Oceanographic Data Center (NODC) Physical and Chemical Oceanographic Station Data Punch Card also are contained in this manual. This is the standard format used at the NODC; it is intended also for use by other agencies or contributors interested in furnishing oceanographic data to the Data Center or making their data systems compatible with those of NODC. The card format and codes described in this manual have resulted from comments and suggestions by the oceanographic community. Its main features are based on the report of the Eastern Pacific Oceanic Conference (EPOC) Committee on Machine Processing for Oceanographic Data, the International Council for the Exploration of the Sea (ICES) oceanographic station card format, the Canadian Oceanographic Data Center (CODC), and the card format formerly used at the U. S. Navy Hydrographic Office.

The card used by NODC is the 80-column Hollerith punch card. Each column contains ten numbers $(0-9)$ and two additional places above the $O$, designated as the $X$ and $Y$ overpunches. Only one number is used in each column; $X$ and $Y$ are used in combination with numbers to produce alphabetic codes or to denote various special numeric indicators. 
GENERAI

Three basic oceanographic station data cards are in use currently at NODC. These are the Master Card, the Observed Detail Card, and the Standard Detail Card. Actually, a single, multi-purpose card is used on which the three headings are printed; the identification of the card and the headings to be used are indicated by a control punch in Column 80. The function of each card is as follows: (1) a single Master Card is used to identify each oceanographic station and to record general surface environmental and meteorological information at the station; (2) a separate Observed Detail Card is punched for each depth at which chemical and physical data were taken; and (3) a Standard Detail Card may be punched for standard depths with interpolated physical and chemical data; usually this card is produced automatically by the IBM 7070/1401 Computer System for a.1 standard depths.

The standard multi-purpose card also is used to record the results of the various computations programmed by NODC on the IBM 7070; when cards bear computed values they are referred to as Computed Cards; before computation they are called Data Cards. Additional types of cards are currently under development for recording biological, geological, and additional chemical information observed at the station.

Each oceanographic cruise or source of oceanographic station data processed by NODC is assigned a "reference identity number." NODC Publication C-1, "Reference Sources for Oceanographic Station Data," gives the bibliographic and other pertinent information for each cruise 
by reference identity number. A cruise master card bearing information common to the cruise as a whole, as well as codes indicating observational techniques and accuracy, eventually will be provided for each cruise. The format of this card is now being developed by NODC on the basis of comments and suggestions received from the oceanographic community.

To facilitate card punching, all data are coded or transcribed onto the NODC "Physical and Chemical Data Form for Oceanographic Stations," NODC form NHO-NODC-3167/1 (9-61), which contains space for coding the station Master Card information and for coding physical and chemical information at 25 observed depths. The entries on this form are arranged in the same order as they appear on the punch card (decimal places are marked by dots). The shaded portion of the form is for information to be filled in by NODC only. Some columns are left out on the portion of the form which corresponds to the detail card; these data are always machine reproduced or computed and do not need to be filled in by the observer or coder.

\section{CODING THE MASTER CARD INFORMATION}

General instructions for entries on the data form:

1. Use standard rounding procedures whenever rounding is necessary.

$$
\begin{aligned}
\text { Example: }> & 5 \text { add one (1) to preceding column } \\
< & 5 \text { drop } \\
& 5 \text { round to nearest even number }
\end{aligned}
$$


2. When necessary, $O^{\prime}$ s are prefixed to fill a field but do not need to be suffixed.

\author{
Example: A temperature of $6.3^{\circ} \mathrm{C}$. should be \\ recorded and punched in Columns 33-37 \\ as 06.3 with Columns 36 and 37 left \\ blank and unpunched.
}

ENTRIES FOR SURFACE ENVIROMMENTAL INFORMATION (MASTER CARD)

(See Appendix I, page 105, for sample Physical and Chemical Data Form.) COINMNS 1 and 2 COUNIRY CODE

Enter the IGY country code shown in Table 1. Regardless of the ship's registry, country code should reflect the nationality of the agency sponsoring or operating the vessel for the particular cmise being coded.

COLUMNS 3 and 4 SHIP CODE Enter the first two letters of the ship's name (eg. AL = ALBATROSS, DI $=$ DISCOVERY, HS = HUGH M. SMITH). Duplication of ship letters within any one country code must be avoided (e.g. U. S. AIBATROSS = AI, U. S. AIBACORA = AB). If ship is unknown, leave Columns 3 and 4 blank. COLUMNS $5-9$ IATITUDE

Enter latitude in degrees and minutes. Enter tenths of minute, when available, in Column 9. Table 2 converts seconds to tenths of a minute. Enter $\mathbb{N}$ or $S$ in the space provided on the data form.
COLUMNS $10-15$
IONGITUDE

Enter longitude in degrees and minutes. Enter tenths of minute, when available, in Column 15. Table 2 converts seconds to tenths of a minute. Enter $E$ or $W$ in the space provided on the data form. 
This number will be machine computed if not given. A Marsden Square Chart is provided as Table 3 for convenience in locating the station positions according to the Marsden Square system.

Note on Columns 5-18: For station position use lacation as determined on arrival at oceanographic station whenever possible. Indicate appreciable drift during the time station was occupied by an entry in the Remarks space and a red dash in Column 16.
COLUMINS $19-20$
YEAR

Enter last two digits of year as determined by Greenwich Mean Time (CMP).

$$
\text { COLUMNS } 21-22 \text { MONTH }
$$

Enter month as determined by GMT, using Arabic numerals ol through 12.

$$
\text { COLIMINS } 23-24 \text { DAY }
$$

Enter day of month as determined by GMT. Prefix 0 if less than 10.

$$
\text { COLUMNS } 25-27 \text { TIME }
$$

Enter hour and tenths of hour of time of station in CNI. Use either time of first cast or time at which applicable surface environmental data were obtained. Table 4 converts local time to GNT and Table 2 converts minutes to tenths of an hour.

$$
\text { COLUMNS } 28-30 \quad \text { SHIP'S CRUISE NUMRER }
$$

Enter the number, alphabetic or alpha-numeric designator or its closest equivalent, assigned to the cruise by the originator. Leave blank if none. Example: MRL $60=060$

$$
\text { COLUMISS } 31-33
$$

SHIP'S STATION NUMBER

Enter ship's station number as assigned by the originator. When complex station numbering systems are employed (such as station numbers containing 
positional codes) which require more than 3 columns to record, use the additional columns in the Special Observations field, Columns 68-72. In this case, put a red dash in Column 68.

$$
\text { COLUMNS } 34-37 \quad \text { DEPTH TO BOTTOM }
$$

Enter corrected or uncorrected soundings (as determined at the location given in Columns 5-15) in meters. For corrected soundings, put a red dash in Column 37. Table 5 converts fathoms to meters; Table 6 converts feet to meters. Although Table 6 is given to tenths of a meter for added accuracy in recording observed depths in the detail card, depth to bottom, Columns $34-37$, is rounded to the nearest whole meter.

$$
\text { COLIMINS } 38-39 \text { MAXIMUM SAMPIE DEPTH }
$$

Enter the depth of the deepest sample (temperature or salinity or both) to the nearest hundred-meter interval. Round as follows: $0-50=00$, $51-150=01,151-250=02$, etc.

COLUNNS $40-41$

ADDITIONAL OBSERVATIONS CODE

A two digit code to indicate various kinds of additional data collected at the station but not recorded on the Master or Detail Card is to be entered in this space. The code currently is under development; when completed it will be issued as Table 7 .

$$
\text { COLUMNS } 42-45
$$

WATER COLOR AND TRANSPARENCY

Enter water color in Columns 42 and 43 according to the Forel-Ule scale. Table 8 shows the Forel-Ule scale and conversions from other color scales. Enter transparency values in whole meters in Columns 44 and 45 obtained by using a white, $30 \mathrm{~cm}$. Secchi disc. 
Enter the direction from which the dominant waves come in Columns 46 and 47 according to WMO Code 0885 (23B)* as shown in Table 9. (If wave height is 16 feet or greater, 50 must be added to code value of direction.) Enter the height of the dominant waves in Column 48 according to WMO Code 1555 (42)* shown in Tabie 11. (If Sea state is given, see note below.) Enter the period of the dominant waves in Column 49 according to WMo Code 3155 (69)* as shown in Table 12.

To record Sea State instead of wave height and period, enter the state of sea (or amount) in Column 48 according to WMO Code 3700 (75)" as shown in Table 19 and cross out H in Column 48 ; leave Column 49 blank.

$$
\text { COLUMNS } 50-53 \text { WIND DIRECTION AND SPEED (OR FORCE) }
$$

Enter direction from which the wind blows in Columns 50 and 51 according to WMO Code 0877 (23)* as shown in Table 9. Table 10 is for conversion from points, quarter points, or a scale of 32. There is a cholce of either wind speed or force in this field:

1. Wind speed in knots. Conversions are given in:

$$
\begin{aligned}
& \text { Table } 14 \text { (meters/second to knots) } \\
& \text { Table } 15 \text { (m1les/hour to knots) } \\
& \text { Table } 16 \text { (k1lometers/hour to knots) } \\
& \text { Table } 17 \text { (feet/second to knots) }
\end{aligned}
$$

2. Wind force according to the Beaufort scale as defined by Table 18. (Do not use WMO Code 1144 (30)*.)

Important: the unit not used, i.e. speed or force, should be crossed off the data form.

* Number in parentheses represents old WMO Code number. 
Enter barometric pressure in millibars. Enter tens, units, and tenths only. (Example: $1012.62=12.6$.$) The recordable range is 945.0$ to 1044.9 mbs. Enter pressure falling outside this range in the Remarks space of the data form. Table 19 converts inches to millibars. Table 20 converts millimeters to millibars.

$$
\text { COLUMNS } 57-62
$$

Enter dry bulb temperature in Columns 57-59 and wet bulb temperature in Columns $60-62$ in ${ }^{\circ} \mathrm{C}$ to tenths. Indicate negative temperature by a prominent red dash over the numeral(s) in Column(s) 57 and/or 60. Table 21 converts ${ }^{\circ} \mathrm{F}$ to ${ }^{\circ} \mathrm{C}$.

$$
\text { COLIMMNS } 63-64
$$

WEATHER

There is a choice of two types of entries for weather:

1. Enter an $X$ in Column 63 and enter the weather in Column 64 according to the single digit WMO Code 4501 (90A)* as shown in Table 22. This is the preferred weather code.

2. Enter present weather in Columns 63 and 64 according to the two digit WMO code 4677 (92)* as shown in Table 25. Because code figures 00 through 03 are not descriptive of present weather, code 4501 is preferred instead of these code figures. Conversions from other weather codes are given in Tables 23 and 24.

*Number in parentheses represents old WMO Code number. 
Enter type (genus) of cloud according to wMo Code 0500 (10)* as shown in Table 26.

COLUMN 66

CLOUD AMOUNT

Enter cloud amount (fraction of the sky covered by clouds) in elghths according to WMO Code $2700(60) *$ as shown in Table 27. COLIMN 67 VISIBILITY

Enter visibility according to WMO Code 4300 (85A)* as shown in Table 28. COLUMNS $68-72$ SPECIAL OBSERVATIONS

Enter special observations in this space. Specify nature of special observations, units, codes, and columns used in the Remarks space of the data form. As mentioned previously, these columns also may be used for ship's station numbers that exceed 3 digits (see page 5, Columns 31-33, SHIP'S STATION NUMBER). However, when used for this purpose, no other information may be placed in this field.

$$
\text { COLUNNS } 73-80
$$

PROCESSING NUMBERS

(TO BE COMPLETED BY NODC)

The reference identity number assigned by NODC is entered in Columns $73-75$.

NODC assigns numbers to each station consecutively in the order in which they appear in the source. These "consec numbers" are entered in Columns 76-79. NODC enters one (I) In Column 80 to identify the station Master Card.

* Number in parentheses represents old WMO Code number. 
General instructions for entries on the data form:

1. Columns 32,37 , and 42 , marked i, may be used for the following purposes:

a) For insertion of an additional decimal place, if needed, or

b) For insertion of a "precision of measurement" indicator. An alphabetic code, applicable to depth, temperature, and salinity, will indicate the estimated limits of accuracy determined by the observer. This code, presently under development, will be distributed as Table 29. (Columns 32, 37 , and 42 cannot be used simultaneously for any one cruise or station for both addit1onal decimal pleces and precision of measurement indicator.)

2. To indicate doubtful depth, temperature, and salinity place a question mark (?) in the indicator columns (i.e., Columns 32, 37, and 42) or in the case of oxygen and optional chemistry after the last numeral of the value in the last column of the field. The last column of each field has been widened on the data form to allow for the possible entry of both a numeral and a question mark. 
ENTRIES FOR SUBSURFACE OBSERVATIONS (DETAII CARD)

(See Appendix I, page 105, for sample Physical and Chemieal Data Form.) COLUMNS $1-24$

IDENIIFICATION ENTRIES

These columns are identical to the Master Card and need not be filled in. The information is automatically reproduced in the Observed Detail Card from the Master Card.

$$
\text { COLUMNS } 25 \text { - } 27 \quad \text { MESSENGER TIME }
$$

Enter hour and tenths of hour in GMT at time of release of messenger applicable to the observational level. Table 2 converts minutes to tenths of an hour. The time entry also serves to distinguish multiple casts at one station. If a single cast constitutes the station, the messenger time need be entered only at the shallowest (0-meter) level.

$$
\text { COLUMNS } 28-32 \quad \text { DEPTH OF SAMPLE }
$$

Enter depth of a sample in meters in Columns $28-31$. Column 32 may be used to enter tenths of a meter, if desired, or the alphabetic precision indicator (see general instructions). To indicate thermometrically determined depths place a cross $(\dagger)$ in Column 32. The entries in the depth field should be corrected depths only. Standard depths are normally interpolated by the computer and need not be entered on the data form.

If interpolated standard depths are entered, leave messenger time blank and insert a 7 in Column 80.

$$
\text { COLUMNS } 33-37 \quad \text { TEMPERATURE }
$$

Enter temperature in ${ }^{\circ} \mathrm{C}$ to hundredths in Columns 33-36; Column 37 is for an additional decimal or precision indicator coded according to Table 29. To indicate a negative temperature, place a prominent minus sign in red before the numeral entry in Column 33. (Column 33 has extra width for this purpose.) 
Enter salinity in parts per thousand to hundredths in Columns 38-41; Column 42 is for an additional (thousandths) decimal. Table 30 converts chlorinity to salinity.

Columns 48-50 do not appear on the data form. On the punch card these columns are reserved for computed sigma-t and sound velocity. Sound velocities measured by a velocimeter for temperatures or salinities based on these measurements) should be forwarded or coded separately.

$$
\text { COLUMINS } 51-53
$$

OXYGEN

Enter oxygen in milliliters per liter $(\mathrm{ml} / \mathrm{l})$ to hundredths in Columns 51-53. (If determined to tenths only leave Column 53 blank.) Table 31 converts milligrams per liter (mg/l) and Table 32 converts milligramatoms per liter $(\mathrm{mg}-\mathrm{at} / \mathrm{l})$ to $\mathrm{ml} / \mathrm{l}$.

To record oxygen values greater than $9.99 \mathrm{ml} / \mathrm{l}$ enter two numerals (tens and units) in Column 51.

$$
\text { COLUMNS } 54-71
$$

OPTIONAL CHEMISTRY

Columns 54-71 are intended for entry of the additional chemistry described below; this is the only chemistry that will be punched routinely. A special punch card to record chemistry other than that shown below is being developed at NODC. However, any chemistry may be substituted in these columns by special request provided that:

1. It can be reported in a 3 digit field.

2. The nature and units of the substituted chemistry and the columns used are fully described in the Remarks space of the data form. 
Enter inorganic phosphate in microgram-atoms per liter ( $\mathrm{ug-a.t/I)}$ to hundredths. Conversions from other units are shown in Tables 33-35. COLUMNS 57 - 59 TOTAL PHOSPHORUS

Enter total $\mathbf{P}$ in microgram-atoms per liter ( $\mu$ g-at/l) to hundredths. COLIMNS $60-62$ NITRITE-NITROGEN

Enter nitrite-nitrogen $\left(\mathrm{NO}_{2}-\mathrm{N}\right)$ in microgram-atoms per liter (ug-at/I) to hundredths. Conversions from micrograms per liter of $\mathrm{NO}_{2}$ to microgram-atoms per liter of $\mathrm{NO}_{2}-\mathrm{N}$ are shown in Table 36.

$$
\text { COLUMNS } 63-65 \text { NITRATE-NITROGEN }
$$

Enter nitrate-nitrogen $\left(\mathrm{NO}_{3}-\mathrm{N}\right.$ ) in microgram-atoms per liter (ug-at/I) to tenths. Conversions from micrograms per liter of $\mathrm{NO}_{3}$ to microgramsatoms per liter of $\mathrm{NO}_{3}-\mathrm{N}$ are shown in Table 37 .

$$
\text { COLUMNS } 66-68 \text { SILICATE-SILICON }
$$

Enter silicate-silicon in microgram-atoms per liter ( $\mu g-a t / 1$ ). Conversions from other units are shown in Tables 38-40.

$$
\text { COLUMNS } 69-71 \quad p^{\mathrm{H}}
$$

Enter $p^{H}$ in Columns 69-71.

COLUMN 72

This column (reserved for NODC) is to be left blank.

Columns 73-79 are omitted from the data form; on the punch card these will be reproduced automatically from the Master Card to provide each Detail Card with reference identity and consecutive numbers. 
For observed sample depths enter 3 in Column 80 to indicate an Observed Detail Card. If interpolations are made by non-machine methods for standard depths enter a 7 in Column 80 . (Computed elements such as sigma-t, sound velocity, dynamic depth anomaly, etc. will be computed for this type of card, but computations will be based on the temperature and salinity as interpolated by the originator.)

In a few instances only interpolated data may be available for certain stations. In that case the numeral 4 is entered in Column 80. Such a card will be treated by the computer as though it were an observed level. Stations based on such interpolated data will be appropriately marked "LIT" on listings. 
INSTRUCTIONS FOR PUNCHING NODC OCEANOGRAPHIC STATION CARDS

FROM THE PHYSICAL AND CHEMICAL DATA CODING FORM

(See Appendix I, page 105, for sample Oceanographic Station Card.)

In general the physical and chemical data form for oceanographic station data is arranged in such a manner that most of the numeric and alphabetic entries can be punched directly into the corresponding columns of the punch card. The alphabetic punch code used at NODC is the standard IBM code. The correct entry columns for certain overpunches, however, cannot readily be determined from the data form. The correct entry for these overpunches is given in the punching instructions below.

\section{MASTER CARD}

EINTRY ON DATA FORM

Entry s between Columns

9 and $\overline{10}$.

Entry E between Columns

15 and 16 .

Red dash in Column 16

Red dash in Column 37

Letter $\mathrm{H}$ crossed out in

Column 48 (A not crossed

out) and no entry in Column 49

Entry $X$ in Column 48

Entry $\mathrm{X}$ in Column 49

Word SPEFD crossed out over

Columns 52 and 53 (word

FORCE not crossed out)
ENTRY IN PUNCH CARD (OVERPUNCHES UNLESS STATED OTHERWISE)

$\mathrm{x}$ in Column 8

$\mathrm{x}$ in Column 14

$\mathrm{x}$ in Column 15

$\mathrm{x}$ in Column 37

$x$ in Column 47

Punch alphabetic X in Column 48

Punch alphabetic $X$ in Column 49

$x$ in Column 52 
Red dash in Column 57

Red dash in Column 60

Letter in Column 63

Entry $x$ in Column 65

Red dash in Column 68

Card Type $x$ in Column 59

$x$ in Column 62

Punch alphabetic $X$ in Column 63

Punch alphabetic $\mathrm{x}$ in Column 65

$x$ in Column 68

Enter 1 in Column 80

\section{DETAII CARD}

CODING FORM

Columns $1-24$

Question mark (?) In

Column 32

Cross ( $t$ ) in Column 32

Red dash in Column 33

Question mark (?) in

Column 37

Question mark (?) in

Column 42

Two (2) numerals in

Column 51

Question mark (?) in

Column 53

Columns $73-79$

\section{PUNCH CARD}

Reproduced from Master Card

Q in Column 32

$x$ in Column 28

$x$ in Column 36

Q in Column 37

Q in Column 42

$x$ in Column 51

$\mathrm{x}$ in Column 53

Reproduced from Master Card 
An outline of the format of the Computed Card is included in this manual in order to acquaint the users of the NODC ocennographic station data holdings with the end product of the routinely performed computations. (A detailed description of formulas and techniques used for interpolations and computation of various parameters will be described in a future NODC publication in the General Series.)

The manually punched cards described in the previous section are used as data cards for the NODC computer. The end product of the computation process is a new set of cards referred to as the Computed Cards. There are two types of Computed cards. One type contains computed parameters as well as the original observed information. The other type contains computed values only and is generated for all. standard depths; this card carries the interpolated values of temperature, salinity, and oxygen as well as certain additional parameters which are computed for standard depths only.

The routinely computed parameters which appear on each type of Computed Card are as follows:

NAME OF CARD TYPE

Computed Master Card*

Computed Observed Card*
COMPUTED PARAMETER

Marsden square number

1. Sigma-t $\left(\sigma_{t}\right)$

2. Sound velocity (ft./sec.)

*Code indicators for each card type are given in table on. Page 21. 
1. Interpolated values of temperature, salinity, and oxygen

2. Signa-t $\left(\sigma_{t}\right)$

3. Sound velocity (as above)

4. Specific volume anomaly

5. Dynamic depth anomaly

Additionally, a number of overpunches are included in these cards, some of which are generated during the computation process. A complete list of overpunches which may be used in the basic NODC physical and chemical oceanographic station data Computed Card follows.

*Code indicators for each card type are given in table on Page 21. 
Columns $1-4$

Column 8

Column 14

Column 15

Columns $28-33$

Column 37

Columns $40-41$

Column 47

Column 48

Column 49

Column 52

Column 59

Column 62

Column 63

Column 65
Alphabetic code (possible)

$x=\operatorname{South}($ no $x=$ North)

$x=$ East (no $x=$ West)

$x$ - "extensive" drift while on station

Alphabetic code (possible)

Corrected sounding

Reserve $\mathrm{x}$ and $\mathrm{y}$ code overpunch for Additional Observations Code

$x=$ State of the sea (or sea amount) given rather than wave height and period

Alphabetic code $(X)$ to indicate height of waves not determined

Alphabetic code $(X)$ to indicate calm, or period not determined

$\mathrm{x}=$ Wind force given rather than speed.

$x=$ Negative air temperature (DRY)

$\mathrm{x}=$ Negative air temperature (WET)

Alphabetic code $(X)$ to indicate use of WMO 4501 (90A)*

Alphabetic code $(X)$ to indicate clouds not visible owing to darkness, fog, etc.

* Number in parentheses represents old WMO Code number. 
$x=$ Entry in Columns 68-72 is continuation of Shlp's

Station Number field.

Column $78 *$

$\mathrm{x}=$ Geographical sorted deck indicator

Column 79*

$\mathrm{x}=$ Computed deck indicator

COMPUTED OBSERVED CARD (CODE 3 OR 4 IN COLUMN 80)

Columns $1-24$

Column 28

Column 32

Column 36

Column 37

Column 42

Column $46 *$

Column $47^{*}$

Column 51

Column 53
Reproduced from Master Card

$\mathrm{x}=$ Thermometric depth

Alphabetic code for precision indicator. $Q$ indicates doubtful depth.

$\mathrm{x}=$ Negative temperatures

Alphabetic code for precision indicator. $Q$ indicates doubtful temperature.

Alphabetic code for precision indicator. Q indicates doubtful salinity.

$\mathrm{x}=$ Negative sigma-t $\left(\sigma_{t}\right)$

$x=\operatorname{Add} 5000$ to value in Columns 47-50

No $\mathrm{x}=$ Add 4000 to value in Columns 47-50

$x=$ Add 10 to $O_{2}$ value

$x=$ Doubtful

* Overpunch generated during the computation process. 
Columns $1-53$

Column 59*

Column 63*
Same as Observed Detail Card

$x$ - Negative specific volume anomaly

$\mathrm{x}=$ Negative dynamic depth anomaly

CARD TYPE IDENTIFICATION CODE
CARD TYPE

Master

Observed

NODC Computed Standard

Originator's Computed Standard
CODE IN COLUMN 80

1

3 or $4 * *$

6

7

*Overpunch generated during the computation process

* When only originator's computed standard values are available, a 4 is placed in Column 80. This card will act as an Observed Card in the NODC computer; i.e., it may carry optional chemistry. In addition, NODC Computed Standard Cards (type 6) will be computed. 

TABLE 1

Country Code

International Geophysical Year (IGY) Code

CODE

$\underline{\text { NAME }}$

$\mathrm{OI}$

08

09

10

11

23

13

14

84

83

85

82

15

12

16

18

19

20

21

22

81

26

70

28

27

75

32

33

34

35

17

02

30

25

87

03

Afghanistan

Argentina.

Australia

Austria.

Belgium

Belgian Congo

Bolivia.

Brazil

British Caribbean Territories

British East African Territories and

Indian Ocean Islands

British Malaya/Borneo Territories

British West African Territories

Bulgaria

Burma

Cambodia

Canada

Ceylon

Chile

China.

Columbia

Czechoslovakia

Denmark

Dominican Republic

Ecuador

Egypt

El Salvador

Ethiopia

Federation of Rhodesia and Nyasaland

Finland

France

French Cameroons

French Equatorial Africa

French Oceania

French Somaliland

French Togoland

French West Africa 
TABLE 1 (Cont ${ }^{t} d$ )

\begin{tabular}{|c|c|}
\hline CODE & $\underline{\text { NAME }}$ \\
\hline 06 & Germany \\
\hline 36 & Greece \\
\hline 37 & Guatemala \\
\hline 38 & Haiti \\
\hline 39 & Hong Kong \\
\hline 40 & Hungary \\
\hline 46 & Iceland \\
\hline 41 & India \\
\hline 42 & Indonesia \\
\hline 44 & Iran \\
\hline 43 & Iraq \\
\hline 45 & Ireland \\
\hline 47 & Israel \\
\hline 48 & Italy \\
\hline 49 & Japan \\
\hline 50 & Jordan \\
\hline 24 & Korea \\
\hline 51 & Laos \\
\hline 52 & Lebanon \\
\hline 53 & Libya \\
\hline 54 & Luxembourg \\
\hline 55 & Madagas car \\
\hline $\begin{array}{l}56 \\
57\end{array}$ & Morocco \\
\hline 21 & Mexico \\
\hline 64 & Netherlands \\
\hline 07 & Netherlands Antilles \\
\hline 60 & Netherlands New Guinea \\
\hline 59 & New Caledonia \\
\hline 61 & New Zealand \\
\hline 58 & Norway \\
\hline 62 & Pakistan \\
\hline 63 & Paraguay \\
\hline 72 & People's Republic of Albania \\
\hline 65 & Peru \\
\hline 66 & Philippines \\
\hline 67 & Poland \\
\hline 68 & Portugal \\
\hline 05 & Portuguese East Africa \\
\hline 04 & Portuguese West Africa, \\
\hline
\end{tabular}


TABIE 1 (Cont' $d$ )

\begin{tabular}{ll} 
CODE & \multicolumn{1}{c}{ NAME } \\
73 & Romania \\
29 & Spain \\
76 & Sudan \\
79 & Surinam \\
77 & Sweden \\
78 & Switzerland \\
80 & Syria \\
86 & Thailand \\
88 & Tunisia \\
89 & Turkey \\
91 & Union of South Africa \\
90 & Union of Soviet Socialist Republics \\
94 & United Kingdom of Great Britain \\
74 & and Northern Ireland \\
31 & United States of America \\
92 & Uruguay \\
93 & Venesuela \\
94 & Viet-Nam \\
95 & Yugoslavia
\end{tabular}

NOTE: 69 and 71 have not been assigned. 
TABIE 2

Tenths Conversion

Conversion from seconds (of position) or minutes (of time) to tenths of minutes or hours

\begin{tabular}{|c|c|}
\hline $\begin{array}{c}\text { Range of } \\
\text { Secs. or Mins. }\end{array}$ & $\begin{array}{c}\text { Tenths of } \\
\text { Mins. or Hrs. }\end{array}$ \\
\hline $00-05$ & 0 \\
$06-11$ & 1 \\
$12-17$ & 2 \\
$18-23$ & 3 \\
$24-29$ & 4 \\
$30-35$ & 5 \\
$36-41$ & 6 \\
$42-47$ & 7 \\
$48-53$ & 9 \\
$54-59$ & \\
\hline
\end{tabular}




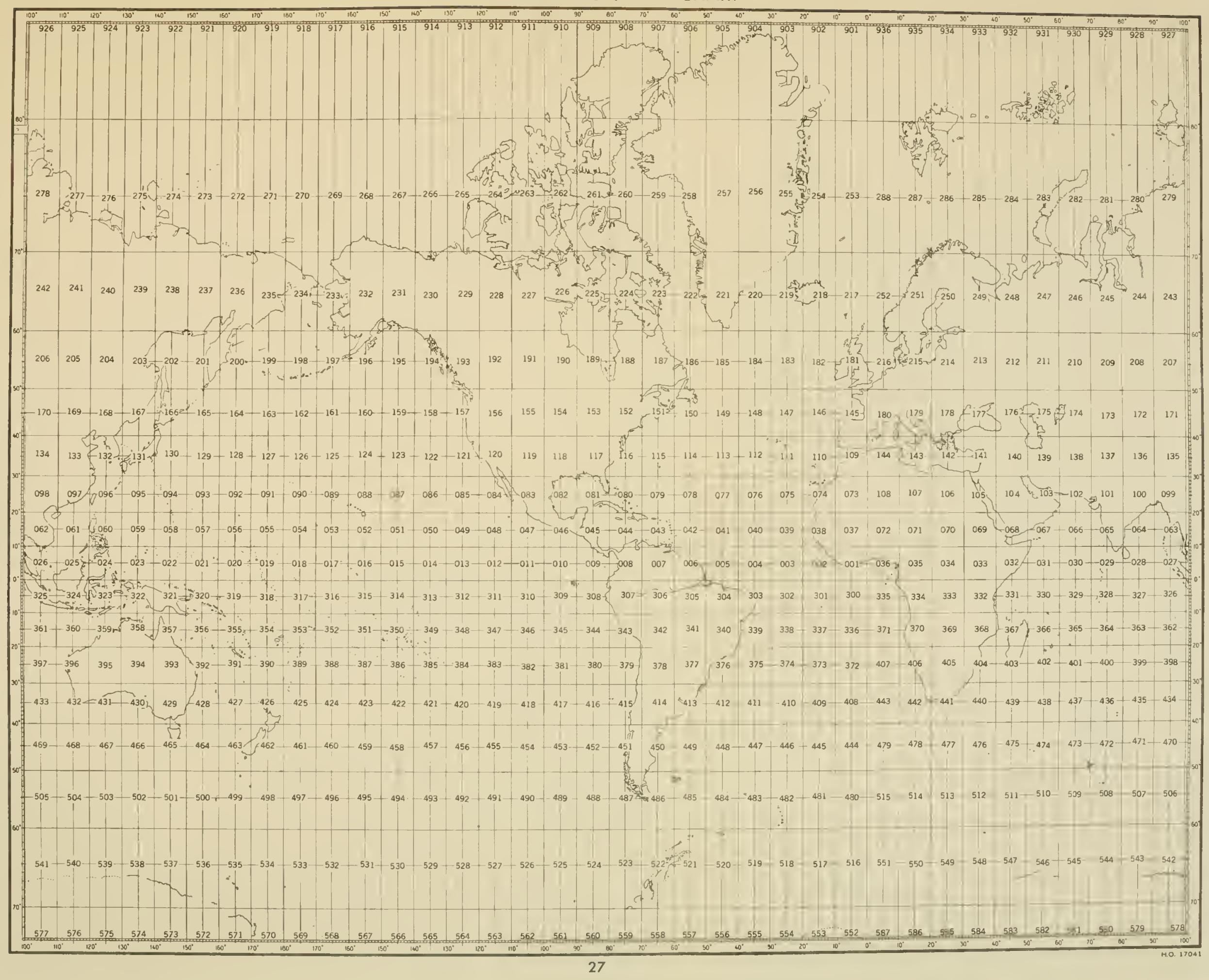



FOLLOIVING DAY

鹪

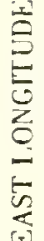

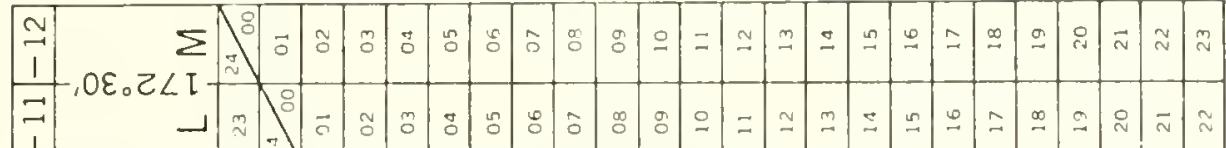

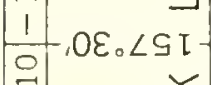

$\therefore \quad \therefore \approx \approx$ ㅇำ o. OE。ट⿱ $\mathrm{I}$

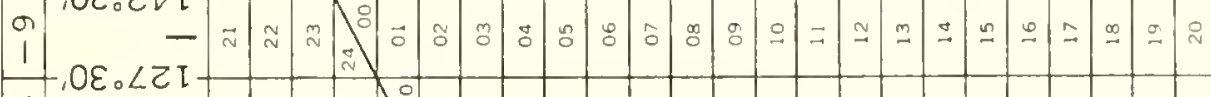

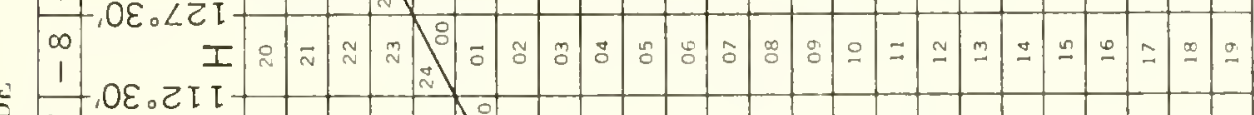

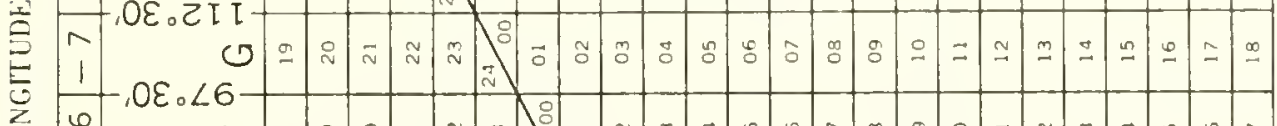

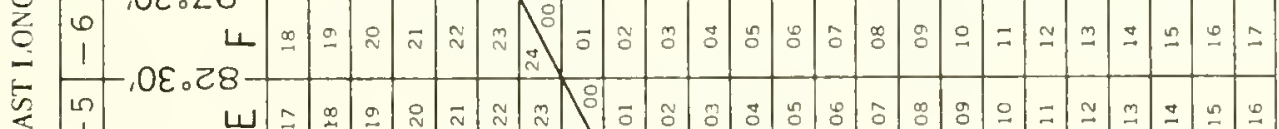

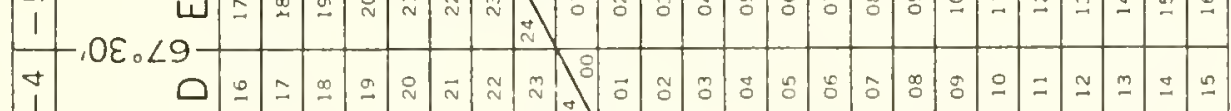

$\frac{1}{m}, 0 \varepsilon .25$

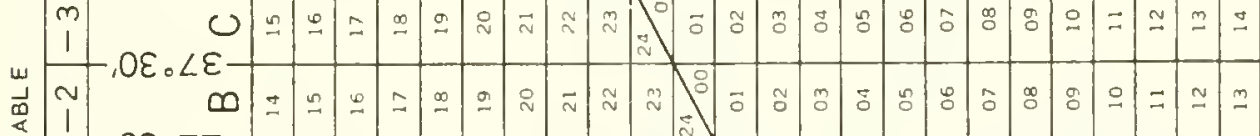

$\stackrel{a}{⺊}$

z $\rightarrow 0 \varepsilon_{0}$ ¿ट

离 , $0 \varepsilon_{0} \angle O-$

$\frac{1}{2}$

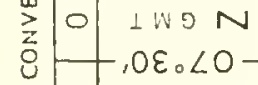

岁

㞱

0

$0 \div=$

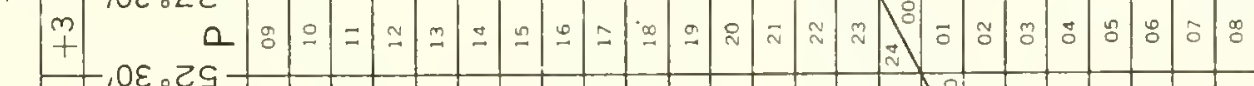

in.

(a)

$-$

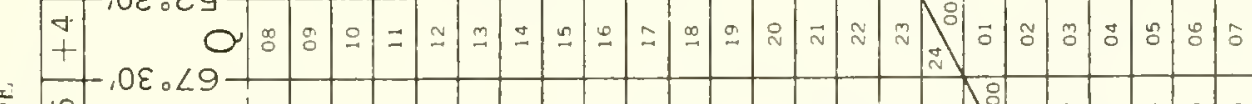

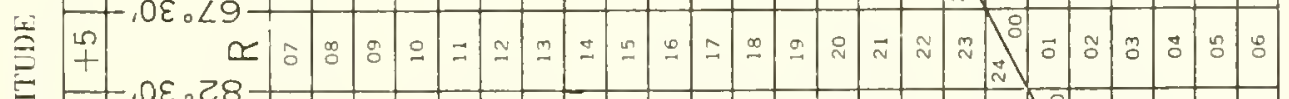

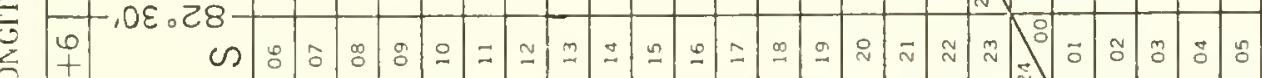

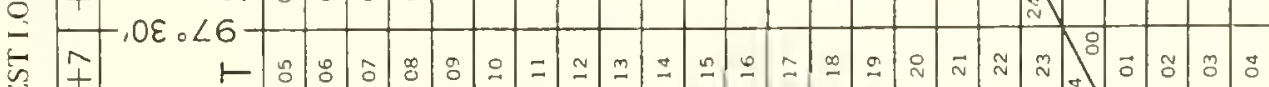

2

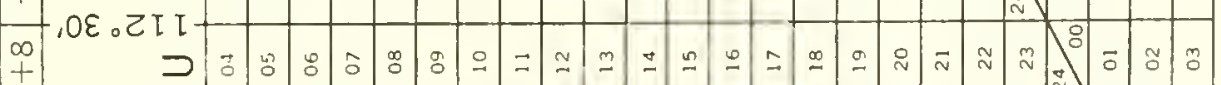

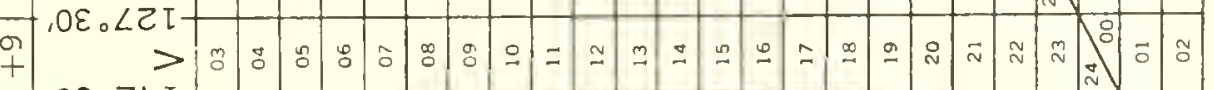

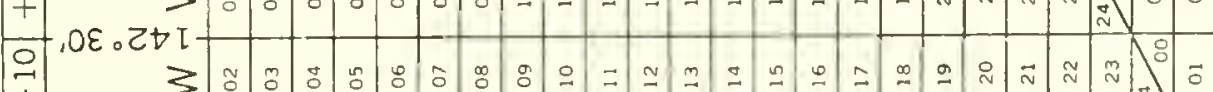

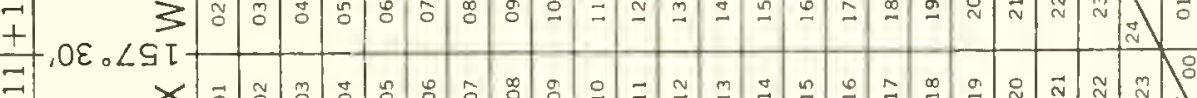

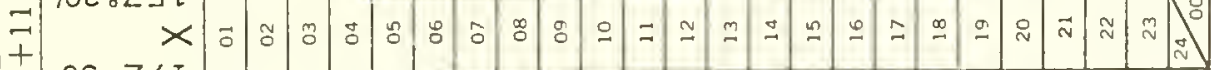

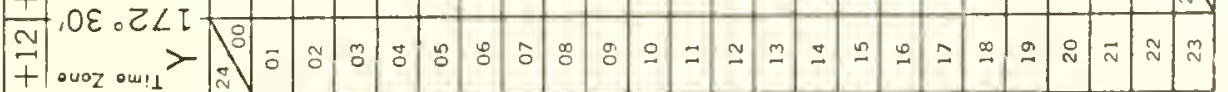


Depth

Conversion from fathoms to meters

( 1 fathom $=1.8288$ meters)

\begin{tabular}{|c|c|c|c|c|c|c|c|c|c|c|}
\hline Fathoms & 0.0 & 0.1 & 0.2 & 0.3 & 0.4 & 0.5 & 0.6 & 0.7 & 0.8 & 0.9 \\
\hline Meters & 0 & 0 & 0 & 1 & 1 & 1 & 1 & 1 & 1 & 2 \\
\hline Fathoms & 0 & 1 & 2 & 3 & 4 & 5 & 6 & 7 & 8 & 9 \\
\hline 00 & 0000 & 0002 & 0004 & 0005 & 0007 & 0009 & 0011 & 0013 & 0015 & 0016 \\
\hline 10 & 0018 & 0020 & 0022 & 0024 & 0026 & 0027 & 0029 & 0031 & 0033 & 0035 \\
\hline 20 & 0037 & 0038 & 0040 & 0042 & 0044 & 0046 & 0048 & 0049 & 0051 & 0053 \\
\hline 30 & 0055 & 0057 & 0059 & 0060 & 0062 & 0064 & 0066 & 0068 & 0069 & 0071 \\
\hline 40 & 0073 & 0075 & 0077 & 0079 & 0080 & 0082 & 0084 & 0086 & 0088 & 0090 \\
\hline 50 & 0091 & 0093 & 0095 & 0097 & 0099 & 0101 & 0102 & 0104 & 0106 & 0108 \\
\hline 60 & 0110 & 0112 & 0113 & 0115 & 0117 & 0119 & 0121 & 0123 & 0124 & 0126 \\
\hline 70 & 0128 & 0130 & 0132 & 0134 & 0135 & 0137 & 0139 & 0141 & 0143 & 0144 \\
\hline 80 & 0146 & 0148 & 0150 & 0152 & 0154 & 0155 & 0157 & 0159 & 0161 & 0163 \\
\hline 90 & 0165 & 0166 & 0168 & 0170 & 0172 & 0174 & 0176 & 0177 & 0179 & 0181 \\
\hline 100 & 0183 & 0185 & 0187 & 0188 & 0190 & 0192 & 0194 & 0196 & 0198 & 0199 \\
\hline 110 & 0201 & 0203 & 0205 & 0207 & 0208 & 0210 & 0212 & 0214 & 0216 & 0128 \\
\hline 120 & 0219 & 0221 & 0223 & 0225 & 0227 & 0229 & 0230 & 0232 & 0234 & 0236 \\
\hline 130 & 0238 & 0240 & 0241 & 0243 & 0245 & 0247 & 0249 & 0251 & 0252 & 0254 \\
\hline 140 & 0256 & 0258 & 0260 & 0262 & 0263 & 0265 & 0267 & 0269 & 0271 & 0272 \\
\hline 150 & 0274 & 0276 & 0278 & 0280 & 0282 & 0283 & 0285 & 0287 & 0289 & 0291 \\
\hline 160 & 0293 & 0294 & 0296 & 0298 & 0300 & 0302 & 0304 & 0305 & 0307 & 0309 \\
\hline 170 & 0311 & 0313 & 0315 & 0316 & 0318 & 0320 & 0322 & 0324 & 0326 & 0327 \\
\hline 180 & 0329 & 0331 & 0333 & 0335 & 0336 & 0338 & 0340 & 0342 & 0344 & 0346 \\
\hline 190 & 0347 & 0349 & 0351 & 0353 & 0355 & 0357 & 0358 & 0360 & 0362 & 0364 \\
\hline 200 & 0366 & 0368 & 0369 & 0371 & 0373 & 0375 & 0377 & 0379 & 0380 & 0382 \\
\hline 210 & 0384 & 0386 & 0388 & 0390 & 0391 & 0393 & 0395 & 0397 & 0399 & 0401 \\
\hline 220 & 0402 & 0404 & 0406 & 0408 & 0410 & 0411 & 0413 & 0415 & 0417 & 0419 \\
\hline 230 & 0421 & 0422 & 0424 & 0426 & 0428 & 0430 & 0432 & 0433 & 0435 & 0437 \\
\hline 240 & 0439 & 0441 & 0443 & 0444 & 0446 & 0448 & 0450 & 0452 & 0454 & 0455 \\
\hline 250 & 0457 & 0459 & 0461 & 0463 & 0465 & 0466 & 0468 & 0470 & 0472 & 0474 \\
\hline 260 & 0475 & 0477 & 0479 & 0481 & 0483 & 0485 & 0486 & 0488 & 0490 & 0492 \\
\hline 270 & 0494 & 0496 & 0497 & 0499 & 0501 & 0503 & 0505 & 0507 & 0508 & 0510 \\
\hline 280 & 0512 & 0514 & 0516 & 0518 & 0519 & 0521 & 0523 & 0525 & 0527 & 0529 \\
\hline 290 & 0530 & 0532 & 0534 & 0536 & 0538 & 0539 & 0541 & 0543 & 0545 & 0547 \\
\hline
\end{tabular}




\section{TABLE 5 (Cont'd)}

Depth

Conversion from fathoms to meters

( 1 fathom $=1.8288$ meters)

\begin{tabular}{|c|c|c|c|c|c|c|c|c|c|c|}
\hline Fathoms & 00 & 10 & 20 & 30 & 40 & 50 & 60 & 70 & 80 & 90 \\
\hline 300 & 0549 & 0567 & 0585 & 0604 & 0622 & 0640 & 0658 & 0677 & 0695 & 0713 \\
\hline 400 & 0732 & 0750 & 0768 & 0786 & 0805 & 0823 & 0841 & 0860 & 0878 & 0896 \\
\hline 500 & 0914 & 0933 & 0951 & 0969 & 0988 & 1006 & 1024 & 1042 & 1061 & 1079 \\
\hline 600 & 1097 & 1116 & 1134 & 1152 & 1170 & 1189 & 1207 & 1225 & 1244 & 1262 \\
\hline 700 & 1280 & 1298 & 1317 & 1335 & 1353 & 1372 & 1390 & 1408 & 1426 & 1445 \\
\hline 800 & 1463 & 1481 & 1500 & 1518 & 1536 & 1554 & 1573 & 1591 & 1609 & 1628 \\
\hline 900 & 1646 & 1664 & 1682 & 1701 & 1719 & 1737 & 1756 & 1774 & 1792 & 1811 \\
\hline
\end{tabular}

\begin{tabular}{|c|c|c|c|c|c|c|c|c|c|c|}
\hline Fathoms & 000 & 100 & 200 & 300 & 400 & 500 & 600 & 700 & 800 & 900 \\
\hline 1000 & 1829 & 2012 & 2195 & 2377 & 2560 & 2743 & 2926 & 3109 & 3292 & 3475 \\
\hline 2000 & 3658 & 3840 & 4023 & 4206 & 4389 & 4572 & 4755 & 4938 & 5121 & 5304 \\
\hline 3000 & 5486 & 5669 & 5852 & 6035 & 6218 & 6401 & 6584 & 6767 & 6949 & 7132 \\
\hline 4000 & 7315 & 7498 & 7681 & 7864 & 8047 & 8230 & 8412 & 8595 & 8778 & 8961 \\
\hline 5000 & 9144 & 9327 & 9510 & 9693 & 9876 & 10058 & 10241 & 10424 & 10607 & 10790 \\
\hline
\end{tabular}


TABIE 6

Depth

Conversion from feet to meters (tenths)

(I foot $=0.3048$ meter $)$

\begin{tabular}{|c|c|c|c|c|c|c|c|c|c|c|}
\hline Feet & 0 & 1 & 2 & 3 & 4 & 5 & 6 & 7 & 8 & 9 \\
\hline 00 & 0.0 & 0 & 0.6 & 0.9 & & & 1.8 & 2.1 & 2.4 & 2.7 \\
\hline 10 & 3.0 & 3.4 & 3.7 & 4.0 & $+\cdot 3$ & 4.6 & 4.9 & 5.2 & 5.5 & 5.8 \\
\hline 20 & 6.1 & 6.4 & 6.7 & 7.0 & $7 \cdot 3$ & 7.6 & 7.9 & 8.2 & 8.5 & 8.8 \\
\hline 30 & 9.1 & 9.4 & 9.8 & 10.1 & 10.4 & 10.7 & 11.0 & $11 \cdot 3$ & 11.6 & 11.9 \\
\hline 40 & 12.2 & 12.5 & 12.8 & 13.1 & 13.4 & 13.7 & 14.0 & 14.3 & 14.6 & 14.9 \\
\hline 50 & 15.2 & 15.5 & 15.8 & 16.2 & 16.5 & 16.8 & 17.1 & 17.4 & 17.7 & 18.0 \\
\hline 60 & 18.3 & 18.6 & 18.9 & 19.2 & 19.5 & 19.8 & 20.1 & 20.4 & 20.7 & 21.0 \\
\hline 70 & $21 \cdot 3$ & 21.6 & 21.9 & $22 \cdot 3$ & 22.6 & 22.9 & $23 \cdot 2$ & 23.5 & 23.8 & 24.1 \\
\hline 80 & $24 . \overline{4}$ & 24.7 & 25.0 & $25 \cdot 3$ & 25.6 & 25.9 & 26.2 & 26.5 & 26.8 & 27.1 \\
\hline 90 & 27.4 & $27 \cdot 7$ & 28.0 & 28.3 & 28.7 & 29.0 & $29 \cdot 3$ & 29.6 & 29.9 & 30.2 \\
\hline 100 & 30.5 & 30.8 & 31.1 & 31.4 & 31.7 & & $32 \cdot 3$ & 32.6 & 32.9 & 33.2 \\
\hline 110 & 33.5 & 33.8 & 34.1 & 34.4 & 34.7 & 35.1 & 35.4 & 35.7 & 36.0 & 36.3 \\
\hline 120 & 36.6 & 36.9 & 37.2 & 37.5 & 37.8 & 38.1 & 38.4 & 38.7 & 39.0 & 39.3 \\
\hline 130 & 39.6 & 39.9 & 40.2 & 40.5 & 40.8 & 41.1 & 41.5 & 41.8 & 42.1 & 42.4 \\
\hline 140 & 42.7 & 43.0 & $43 \cdot 3$ & 43.6 & $43 \cdot 9$ & 44.2 & 44.5 & 44.8 & 45.1 & 45.4 \\
\hline 150 & 45.7 & 46.0 & 46.3 & 46.6 & 46.9 & 2 & 47.5 & 47.9 & 48.2 & 48.5 \\
\hline 160 & 48.8 & 49.1 & 49.4 & 49.7 & 50.0 & $50 \cdot 3$ & 50.6 & 50.9 & 51.2 & 51.5 \\
\hline 170 & 51.8 & 52.1 & 52.4 & 52.7 & & $53 \cdot 3$ & 53.6 & 53.9 & 54.3 & 54.6 \\
\hline 180 & 54.9 & 55.2 & 55.5 & 55.8 & 56.1 & 56.4 & 56.7 & 57.0 & 57.3 & 57.6 \\
\hline 190 & $57 \cdot 9$ & 58.2 & 58.5 & 58.8 & 59.1 & 59.4 & 59.7 & 60.0 & 60.4 & 60.7 \\
\hline 200 & 61.0 & 61.3 & 61.6 & 61.9 & 62.2 & 62.5 & 62.8 & 63.1 & 63.4 & 63.7 \\
\hline 210 & 64.0 & 64.3 & 64.6 & 64.9 & 65.2 & 65.5 & 65.8 & 66.1 & 66.4 & 66.8 \\
\hline 220 & 67.1 & 67.4 & 67.7 & 68.0 & 68.3 & 68.6 & 68.9 & 69.2 & 69.5 & 69.8 \\
\hline 230 & 70.1 & 70.4 & 70.7 & 71.0 & 71.3 & 71.6 & 71.9 & 72.2 & 72.5 & 72.8 \\
\hline 240 & 73.2 & 73.5 & 73.8 & 74.1 & 74.4 & 74.7 & 75.0 & $75 \cdot 3$ & 75.6 & 75.9 \\
\hline 250 & 76.2 & 76.5 & 76.8 & 77.1 & 77.4 & $77 \cdot 7$ & 78.0 & 78.3 & 78.6 & 78.9 \\
\hline 260 & 79.2 & 79.6 & 79.9 & 80.2 & 80.5 & 80.8 & 81.1 & 81.4 & 81.7 & 82.0 \\
\hline 270 & $82 \cdot 3$ & 82.6 & $82 \cdot 9$ & 83.2 & 83.5 & 83.8 & 84.1 & 84.4 & 84.7 & 85.0 \\
\hline 280 & 85.3 & 85.6 & 86.0 & 86.3 & $\begin{array}{l}86.6 \\
80.6\end{array}$ & 86.9 & $87 \cdot 2$ & 87.5 & 87.8 & 88.1 \\
\hline 290 & 88.4 & 88.7 & 89.0 & 89.3 & & 89.9 & 90.2 & 90.5 & 90.8 & 91.1 \\
\hline
\end{tabular}


TABLE 6 (Cont'd)

Conversion from feet to meters (tenths)

(I foot $=0.3048$ meter)

\begin{tabular}{|c|c|c|c|c|c|c|c|c|c|c|}
\hline Feet & 00 & 10 & 20 & 30 & 40 & 50 & 60 & 70 & 80 & 90 \\
\hline $\begin{array}{l}300 \\
400\end{array}$ & $\begin{array}{r}91.4 \\
121.9\end{array}$ & $\begin{array}{r}94.5 \\
125.0\end{array}$ & $\begin{array}{r}97 . \\
128 .\end{array}$ & 6 & $\begin{array}{l}103.6 \\
134.1\end{array}$ & $\begin{array}{l}06.7 \\
37.2\end{array}$ & 7 & 143 & $\begin{array}{l}115.8 \\
146.3\end{array}$ & 149 \\
\hline $\begin{array}{l}500 \\
600 \\
700 \\
800 \\
900\end{array}$ & $\begin{array}{l}152.4 \\
182.9 \\
213.4 \\
243.8 \\
274.3\end{array}$ & $\begin{array}{l}155.4 \\
185.9 \\
216.4 \\
246.9 \\
277.4\end{array}$ & $\begin{array}{l}158.5 \\
189.0 \\
219.5 \\
249.9 \\
280.4\end{array}$ & $\begin{array}{l}5 \\
.0 \\
5 \\
.0 \\
5\end{array}$ & $\begin{array}{l}164.6 \\
195.1 \\
225.6 \\
256.0 \\
286.5\end{array}$ & $\begin{array}{l}167.6 \\
198.1 \\
228.6 \\
259.1 \\
289.6\end{array}$ & $\begin{array}{l}201.2 \\
231.6 \\
262 \cdot 1 \\
292.6\end{array}$ & $\begin{array}{l}173.7 \\
204.2 \\
234.7 \\
265.2 \\
295.7\end{array}$ & $\begin{array}{l}176.8 \\
207.3 \\
237.7 \\
268.2 \\
298.7\end{array}$ & $\begin{array}{l}1.79 .8 \\
210 . \\
240.8 \\
271 . \\
301.8\end{array}$ \\
\hline $\begin{array}{l}1000 \\
1100 \\
1200 \\
1300 \\
1400\end{array}$ & $\begin{array}{l}304.8 \\
335.3 \\
365.8 \\
396.2 \\
426.7\end{array}$ & $\begin{array}{l}307.8 \\
338.3 \\
368.8 \\
399.3 \\
429.8\end{array}$ & $\begin{array}{l}310.9 \\
341.4 \\
371.9 \\
402.3 \\
432.8\end{array}$ & $\begin{array}{l}313.9 \\
344.4 \\
374.9 \\
405.3 \\
435.9\end{array}$ & $\begin{array}{l}311.0 \\
347.5 \\
408.0 \\
438.9\end{array}$ & $\begin{array}{l}320.0 \\
350.5 \\
381.0 \\
411.5 \\
442.0\end{array}$ & $\begin{array}{l}353.6 \\
384.0 \\
424.5 \\
445.0\end{array}$ & $\begin{array}{l}326.1 \\
356.6 \\
387.1 \\
417.6 \\
448.1\end{array}$ & $\begin{array}{l}329.2 \\
359.7 \\
390.1 \\
420.6 \\
451.1\end{array}$ & $\begin{array}{l}362.7 \\
393.2 \\
423.7 \\
454.2\end{array}$ \\
\hline $\begin{array}{l}1500 \\
1600 \\
1700 \\
1800 \\
1900\end{array}$ & $\begin{array}{l}457.2 \\
487.7 \\
518.2 \\
548.6 \\
579.1\end{array}$ & $\begin{array}{l}460.2 \\
490.7 \\
521.2 \\
551.7 \\
582.2\end{array}$ & $\begin{array}{l}563.3 \\
493.8 \\
524.3 \\
554.7 \\
585.2\end{array}$ & $\begin{array}{l}466.3 \\
496.8 \\
527.3 \\
557.8 \\
588.3\end{array}$ & $\begin{array}{l}469.4 \\
499.9 \\
530.4 \\
560.8 \\
591.3\end{array}$ & $\begin{array}{l}472.4 \\
502.9 \\
533.4 \\
563.9 \\
594.4\end{array}$ & $\begin{array}{l}475.5 \\
506.0 \\
536.4 \\
566.9 \\
597.4\end{array}$ & $\begin{array}{l}478.5 \\
509.0 \\
539.5 \\
570.0 \\
600.5\end{array}$ & $\begin{array}{l}481.6 \\
512.1 \\
542.5 \\
573.0 \\
603.5\end{array}$ & $\begin{array}{l}484.6 \\
515.1 \\
545.6 \\
576.1 \\
606.6\end{array}$ \\
\hline $\begin{array}{l}2000 \\
2100 \\
2200 \\
2300 \\
2400\end{array}$ & $\begin{array}{l}609.6 \\
640.1 \\
670.6 \\
701.0 \\
731.5\end{array}$ & $\begin{array}{l}612.6 \\
643.1 \\
673.6 \\
704.1 \\
734.6\end{array}$ & $\begin{array}{l}615.7 \\
646.2 \\
676.7 \\
707.1 \\
737.6\end{array}$ & $\begin{array}{l}618.7 \\
649.2 \\
679.7 \\
710.2 \\
740.7\end{array}$ & $\begin{array}{l}621.8 \\
652.3 \\
682.8 \\
713.2 \\
743.7\end{array}$ & $\begin{array}{l}624.8 \\
655.3 \\
685.8 \\
716.3 \\
746.8\end{array}$ & $\begin{array}{l}627.9 \\
658.4 \\
688.8 \\
719.3 \\
749.8\end{array}$ & $\begin{array}{l}630.9 \\
661.4 \\
691.9 \\
722.4 \\
752.9\end{array}$ & $\begin{array}{l}634.0 \\
664.5 \\
694.9 \\
725.4 \\
755.9\end{array}$ & $\begin{array}{l}637.0 \\
667.5 \\
698.0 \\
728.5 \\
759.0\end{array}$ \\
\hline $\begin{array}{l}2500 \\
2600 \\
2700 \\
2800 \\
2900\end{array}$ & $\begin{array}{l}762.0 \\
792.5 \\
823.0 \\
853.4 \\
883.9\end{array}$ & $\begin{array}{l}765.0 \\
795.5 \\
826.0 \\
856.5 \\
887.0\end{array}$ & $\begin{array}{l}768.1 \\
798.6 \\
829.1 \\
859.5 \\
890.0\end{array}$ & $\begin{array}{l}771.1 \\
801.6 \\
832.1 \\
862.6 \\
893.1\end{array}$ & $\begin{array}{l}774.2 \\
804.7 \\
835.2 \\
865.6 \\
896.1\end{array}$ & $\begin{array}{l}777.2 \\
807.7 \\
838.2 \\
868.7 \\
899.2\end{array}$ & $\begin{array}{l}780.3 \\
810.8 \\
841.2 \\
871.7 \\
902.2\end{array}$ & $\begin{array}{l}783.3 \\
813.8 \\
844.3 \\
874.8 \\
905.3\end{array}$ & $\begin{array}{l}786.4 \\
816.9 \\
847.3 \\
877.8 \\
908.3\end{array}$ & $\begin{array}{l}109 . \\
819 . \\
850 . \\
880 . \\
911 .\end{array}$ \\
\hline $\begin{array}{l}3000 \\
3100 \\
3200\end{array}$ & $\begin{array}{l}914.4 \\
944.9 \\
975.4\end{array}$ & $\begin{array}{l}917.4 \\
947 \cdot 9 \\
978.4\end{array}$ & $\begin{array}{l}920.5 \\
951.0 \\
981.5\end{array}$ & $\begin{array}{l}923.5 \\
954.0 \\
984.5\end{array}$ & $\begin{array}{l}926.6 \\
957.1 \\
987.6\end{array}$ & $\begin{array}{l}929.6 \\
960.1 \\
990.6\end{array}$ & $\begin{array}{l}932.7 \\
963.2 \\
993.6\end{array}$ & $\begin{array}{l}935.7 \\
966.2 \\
996.7\end{array}$ & $\begin{array}{l}938.8 \\
969.3 \\
999.7\end{array}$ & $\begin{array}{r}94 \\
97 \\
100\end{array}$ \\
\hline
\end{tabular}


TABIE $?$

Additional Observations

This table is to be added later. 
TABLE 8

\section{Water Color}

Forel-Ule scale and conversions from other color scales

\begin{tabular}{|c|c|c|c|}
\hline $\begin{array}{l}\text { Percent } \\
\text { Yellow }\end{array}$ & $\begin{array}{l}\text { Percent } \\
\text { Brown }\end{array}$ & $\begin{array}{c}\text { Forel-Ule } \\
\text { Scale }\end{array}$ & Code \\
\hline $\begin{array}{r}0 \\
2 \\
5 \\
9 \\
14 \\
20 \\
27 \\
35 \\
44 \\
54 \\
65\end{array}$ & $\begin{array}{r}0 \\
2 \\
5 \\
9 \\
14 \\
20 \\
27 \\
35 \\
44 \\
54 \\
65\end{array}$ & $\begin{array}{l}I \\
I I \\
I I I \\
I V \\
V \\
V I \\
V I I \\
V I I \\
I X \\
X \\
X I \\
X I \\
X I I \\
X I V \\
X V \\
X V I \\
X V I I \\
X V I I \\
X I X \\
X X \\
X X I\end{array}$ & $\begin{array}{l}01 \\
02 \\
03 \\
04 \\
05 \\
06 \\
07 \\
08 \\
09 \\
10 \\
11 \\
12 \\
13 \\
14 \\
15 \\
16 \\
17 \\
18 \\
19 \\
20 \\
21\end{array}$ \\
\hline
\end{tabular}


In tens of degrees from which waves and/or winds are coming

$\begin{array}{cc}\text { Code } & \\ 00 & \text { Calm (no waves - no motion) } \\ 01 & 5^{\circ}-14^{\circ} \\ 02 & 15^{\circ}-24^{\circ} \\ 03 & 25^{\circ}-34^{\circ} \\ 04 & 35^{\circ}-44^{\circ} \\ 05 & 45^{\circ}-54^{\circ} \\ 06 & 55^{\circ}-64^{\circ} \\ 07 & 65^{\circ}-74^{\circ} \\ 08 & 75^{\circ}-84^{\circ} \\ 09 & 85^{\circ}-94^{\circ} \\ 10 & 95^{\circ}-104^{\circ} \\ 11 & 105^{\circ}-114^{\circ} \\ 12 & 115^{\circ}-124^{\circ} \\ 13 & 125^{\circ}-134^{\circ} \\ 14 & 135^{\circ}-144^{\circ} \\ 15 & 145^{\circ}-154^{\circ} \\ 16 & 155^{\circ}-164^{\circ} \\ 17 & 165^{\circ}-174^{\circ} \\ 18 & 175^{\circ}-184^{\circ} \\ 19 & 185^{\circ}-194^{\circ} \\ 20 & 195^{\circ}-204^{\circ} \\ 21 & 205^{\circ}-214^{\circ}\end{array}$

Code

22

23

24

25

26

27

28

29

30

31

32

33

$215^{\circ}-224^{\circ}$

$225^{\circ}-234^{\circ}$

$235^{\circ}-244^{\circ}$

$245^{\circ}-254^{\circ}$

$255^{\circ}-264^{\circ}$

$265^{\circ}-274^{\circ}$

$275^{\circ}-284^{\circ}$

$285^{\circ}-294^{\circ}$

$295^{\circ}-304^{\circ}$

$305^{\circ}-314^{\circ}$

$315^{\circ}-324^{\circ}$

$34 \quad 335^{\circ}-344^{\circ}$

$35 \quad 345^{\circ}-354^{\circ}$

$36 \quad 355^{\circ}-4^{\circ}$

49

Waves confused, direction indeterminate (waves equal to or less than $4 \frac{3}{4}$ metres)

$99\left\{\begin{array}{l}\text { Waves confused, direction } \\ \text { indeterminate (waves grea- } \\ \text { ter than } 43 / 4 \text { metres) } \\ \text { Winds variable, or all direct- } \\ \text { ions or unknown }\end{array}\right.$

Table 9 is a combination of WMO Codes 0885 and 0877 . 
TABLE 10

Direction

Conversion from points, quarter points, or a scale of 32 , to a scale of 36 points

\begin{tabular}{|c|c|c|c|c|c|c|c|}
\hline POINTS & $\begin{array}{l}\text { QUARTERR } \\
\text { POINTSS }\end{array}$ & $0-32$ & CODE & POINTS & $\begin{array}{l}\text { QUARTER } \\
\text { POINTS }\end{array}$ & $0-32$ & CODE \\
\hline $\mathbb{N} \times E$ & $\mathrm{~N} 6 \mathrm{E}$ to $\mathrm{N} 4 \mathrm{E}$ & $I$ & 01 & $S \times W$ & $S 6 W$ to $S \geq 4 W$ & 17 & 19 \\
\hline NNE & $\mathrm{N} \perp 5 \mathrm{E}$ to $\mathrm{N} 25 \mathrm{E}$ & 2 & 02 & SSW & $\mathrm{S} 15 \mathrm{~W}$ to S25W & 18 & 20 \\
\hline $\mathrm{NE} \times \mathrm{N}$ & $\mathrm{N} 26 \mathrm{E}$ to $\mathrm{N} 34 \mathrm{E}$ & 3 & 03 & SW $\times S$ & $\mathrm{~S} 26 \mathrm{~W}$ to $\mathrm{S} 34 \mathrm{~W}$ & 19 & 27 \\
\hline \multirow[t]{2}{*}{$\mathrm{NE}$} & $\mathrm{N} 35 \mathrm{E}$ to $\mathrm{N} 45 \mathrm{E}$ & 4 & 04 & SW & S35W to $S 45 \mathrm{~W}$ & 20 & 22 \\
\hline & $\mathrm{N} 46 \mathrm{E}$ to $\mathrm{N} 54 \mathrm{E}$ & & 05 & & $S 46 W$ to $S 54 W$ & & 23 \\
\hline$\sqrt{E} \times E$ & $\mathbb{N} 55 \mathrm{E}$ to $\mathrm{N} 65 \mathrm{E}$ & 5 & 06 & SW $\times$ W & $\mathrm{S} 55 \mathrm{~W}$ to $\mathrm{s} 65 \mathrm{~W}$ & 21 & 24 \\
\hline ENE & $\mathrm{N} 66 \mathrm{E}$ to $\mathrm{N} 774 \mathrm{E}$ & 6 & 07 & WSW & S66W to $S 74 \mathrm{~W}$ & 22 & 25 \\
\hline \multirow[t]{2}{*}{$E \times N$} & N75E to N185E & 7 & 08 & $W \times S$ & $\mathrm{~S} 75 \mathrm{~W}$ to $\mathrm{S} 85 \mathrm{~W}$ & 23 & 26 \\
\hline & $\mathrm{N} 86 \mathrm{E}$ to $\mathrm{N} 89 \mathrm{E}$ & & 09 & & $\mathrm{~s} 86 \mathrm{~W}$ to $\mathrm{S} 89 \mathrm{~W}$ & & 27 \\
\hline \multirow[t]{2}{*}{$\mathrm{E}$} & $E$ & 8 & 09 & W & W & 24 & 27 \\
\hline & $\mathrm{s} 89 \mathrm{E}$ to $\mathrm{S} 86 \mathrm{E}$ & & 09 & & N89W to N86W & & 27 \\
\hline$E \times S$ & $\mathrm{~S} 85 \mathrm{E}$ to $\mathrm{S} 75 \mathrm{E}$ & 9 & 10 & $W \times N$ & $\mathrm{~N} 85 \mathrm{~W}$ to $\mathrm{NT} 5 \mathrm{~W}$ & 25 & 28 \\
\hline ESE & $S 74 \mathrm{E}$ to $\mathrm{S} 66 \mathrm{E}$ & 10 & 11 & WNW & $\mathrm{N} 74 \mathrm{~W}$ to $\mathrm{N} 66 \mathrm{~W}$ & 26 & 29 \\
\hline \multirow[t]{2}{*}{$\mathrm{SE} \times \mathrm{E}$} & S65E to S55E & 11 & 12 & $\mathbb{N W} \times \mathrm{W}$ & $\mathrm{N} 65 \mathrm{~W}$ to $\mathrm{N} 55 \mathrm{~W}$ & 27 & 30 \\
\hline & $S 54 E$ to $S 46 E$ & & 13 & & $\mathrm{~N} 54 \mathrm{~W}$ to $\mathrm{N} 46 \mathrm{~W}$ & & 31 \\
\hline SE & $\mathrm{S} 45 \mathrm{E}$ to $\mathrm{S} 35 \mathrm{E}$ & 12 & 14 & $\mathbb{N W}$ & $\mathbb{N} 45 \mathrm{~W}$ to $\mathrm{N} 35 \mathrm{~W}$ & 28 & 32 \\
\hline$S E \times S$ & $\mathrm{~S} 34 \mathrm{E}$ to $\mathrm{S} 26 \mathrm{E}$ & 13 & 15 & $N W \times N$ & $\mathrm{~N} 34 \mathrm{~W}$ to $\mathrm{N} 26 \mathrm{~W}$ & 29 & 33 \\
\hline SSE & S25E to S15E & 14 & 16 & NNW & $\mathrm{N} 25 \mathrm{~W}$ to $\mathrm{N} 15 \mathrm{~W}$ & 30 & 34 \\
\hline \multirow[t]{2}{*}{$S \times E$} & $\mathrm{~S} 14 \mathrm{E}$ to $\mathrm{S} 6 \mathrm{E}$ & 15 & 17 & $\mathbb{N} \times W$ & $N \perp 4 \mathrm{~W}$ to $N 6 \mathrm{~W}$ & 31 & 35 \\
\hline & S5E to SIE & & 18 & & $\mathrm{~N} 5 \mathrm{~W}$ to $\mathrm{N} I \mathrm{~W}$ & & 36 \\
\hline \multirow[t]{3}{*}{$S$} & $S$ & 16 & 18 & $\mathbb{N}$ & N & 32 & 36 \\
\hline & SIW to S5W & & 18 & & $N \perp E$ to N5E & & 36 \\
\hline & & & & Variable & & & 99 \\
\hline
\end{tabular}




\section{Height}

WMO Code 1555 for recording height of the dominant waves

Code

\begin{tabular}{|c|c|}
\hline \\
\hline \\
\hline & $\begin{array}{l}\text { Less than } 1 / 4 m \\
1 / 2 m\left(1 \frac{1}{2} \mathrm{ft}\right)\end{array}$ \\
\hline & $1 / 2 m\left(1 \frac{1}{2} \mathrm{ft}\right)$ \\
\hline & $2 m\left(6 \frac{1}{2} \mathrm{ft}\right)$ \\
\hline & \\
\hline & $3 \mathrm{~m}(91 / 2 \mathrm{ft})$ \\
\hline & \multirow{2}{*}{ 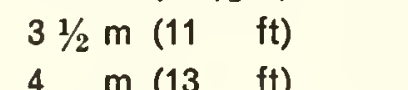 } \\
\hline & \\
\hline & $4 \frac{1}{2} \mathrm{~m}\left(\begin{array}{ll}14 & \mathrm{ft}\end{array}\right)$ \\
\hline
\end{tabular}

\section{Code If 50 is added to direotion}

$$
\begin{aligned}
& \begin{array}{lll}
0 & 5 & \mathrm{~m}
\end{array}\left(\begin{array}{ll}
16 \mathrm{ft} \\
-1
\end{array}\right) \\
& 15 \frac{1}{2} \mathrm{~m}\left(17 \frac{1 / 2 \mathrm{ft})}{}\right. \\
& 26 \mathrm{~m}\left(\begin{array}{ll}
19 & \mathrm{ft}
\end{array}\right) \\
& 3 \quad 6 \frac{1}{2} \mathrm{~m}(21 \mathrm{ft}) \\
& 47 \mathrm{~m}\left(22 \frac{1 / 2 \mathrm{ft})}{}\right. \\
& 5 \quad 7 \frac{1}{2 m}(24 \quad \mathrm{ft}) \\
& 6 \quad 8 \quad m\left(25 \frac{1}{2} \mathrm{ft}\right) \\
& 7 \quad 8 \frac{1}{2} \mathrm{~m}(27 \mathrm{ft})
\end{aligned}
$$

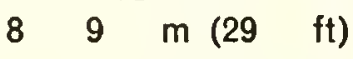

$$
\begin{aligned}
& 9 \quad 91 / 2 \mathrm{~m}(30 \mathrm{1} / 2 \mathrm{ft})
\end{aligned}
$$

Notes:

(1) Each code figure provides for reporting a range of heights. For example: $1=1 / 4 \mathrm{~m}$ $(1 \mathrm{ft})$ to $3 / 4 \mathrm{~m}\left(2 \frac{1}{2} \mathrm{ft}\right) ; 5=2 \frac{1}{4} \mathrm{~m}(7 \mathrm{ft})$ to $23 / 4 \mathrm{~m}(9 \mathrm{ft}) ; 9=4 \frac{1}{4} \mathrm{~m}\left(13 \frac{1}{2} \mathrm{ft}\right)$ to $4 \frac{3}{4} \mathrm{~m}$ $(15 \mathrm{ft})$, etc.

(2) If a wave height comes exactly midway between the heights corresponding to two code figures, the lower code figure is reported; e.g. a height of $23 / 4 \mathrm{~m}$ is reported by code figure 5 .

(3) In aeronautical forecast codes, only the left-hand table is to be used and code figure 9 has the meaning: $4 \frac{1 / 2}{2}(14 \mathrm{ft})$ or more.

(4) The average value of the wave height (vertical distance between trough and crest) is reported, as obtained from the larger well formed waves of the wave system being observed. 


\section{TABIE 12}

\section{Period}

\section{WMO Code 3155 for recording period of dominant waves}

Code

25 seconds or less

36 or 7 seconds

48 or 9 seconds

510 or 11 seconds

612 or 13 seconds

714 or 15 seconds
Code

816 or 17 seconds

918 or 19 seconds

020 or 21 seconds

1 Over 21 seconds

x Calm, or period not determined

Notes:

(1) The period of the waves is the time between the passage of two successive wave crests past a fixed point (it is equal to the wave length divided by the wave speed).

(2) The average value of the wave period is reported, as obtained from the larger well-formed waves of the wave system being observed. 
TABIE 13

Sea State

WMO Code 3700 for Recording Sea State

\begin{tabular}{|l|c|c|c|}
\hline \multirow{2}{*}{ Description } & \multicolumn{2}{|c|}{ Height $(\dagger)$} & \multirow{2}{*}{ Code } \\
\cline { 2 - 3 } & Feet* & Meters & 0 \\
\hline Calm-glassy & 0 & 0 & 1 \\
\hline Calm-rlppled & $0-1 / 3$ & $0-0.1$ & 2 \\
\hline Smooth-wavelet & $1 / 3-1 \frac{2}{3}$ & $0.1-0.5$ & 3 \\
\hline Slight & $1 \frac{2 / 3}{3}-4$ & $0.5-1.25$ & 3 \\
\hline Moderate & $4-8$ & $1.25-2.5$ & 4 \\
\hline Rough & $8-13$ & $2.5-4$ & 5 \\
\hline Very rough & $13-20$ & $4-6$ & 6 \\
\hline High & $20-30$ & $6-9$ & 7 \\
\hline Very high & $30-45$ & $9-14$ & 8 \\
\hline Phenomenal & $>45$ & $>14$ & 9 \\
\hline
\end{tabular}

$(t)$ The average wave height as obtained from the Iarger well-formed waves of the wave system being observed.

* The exact bounding height is to be assigned for the lower code figure, e.g. a height of 4 meters is coded as 5 . 
TABLE 14

Wind Speed

Conversion from meters per second to knots $(1 \mathrm{~m} / \mathrm{sec}-1.94254 \mathrm{knots})$

\begin{tabular}{lllllllllll}
\hline $\mathrm{m} / \mathrm{sec}$ & 0.0 & 0.1 & 0.2 & 0.3 & 0.4 & 0.5 & 0.6 & 0.7 & 0.8 & 0.9 \\
\cline { 2 - 10 } & 0 & 0 & 0 & 1 & 1 & 1 & 1 & 1 & 2 & 2
\end{tabular}

\begin{tabular}{lrrrrrrrrrr}
\hline $\mathrm{m} / \mathrm{sec}$ & \multicolumn{1}{c}{ 00 } & 01 & 02 & 03 & 04 & 05 & 06 & 07 & 08 & 09 \\
\cline { 2 - 10 } 00 & 00 & 02 & 04 & 06 & 08 & 10 & 12 & 14 & 16 & 17 \\
10 & 19 & 21 & 23 & 25 & 27 & 29 & 31 & 33 & 35 & 37 \\
20 & 39 & 41 & 43 & 45 & 47 & 49 & 51 & 52 & 54 & 56 \\
30 & 58 & 60 & 62 & 64 & 66 & 68 & 70 & 72 & 74 & 76 \\
40 & 78 & 80 & 82 & 84 & 85 & 87 & 89 & 91 & 93 & 95 \\
50 & 97 & 99 & 101 & 103 & 105 & 107 & 109 & 111 & 113 & 115
\end{tabular}


TABIE 15

Wind Speed

Conversion from miles per hour to knots

(I $\mathrm{mph}=0.86839 \mathrm{knot}$ )

\begin{tabular}{l|rlllllllll}
\hline mph & 00 & 01 & 02 & 03 & 04 & 05 & 06 & 07 & 08 & 09 \\
\cline { 2 - 10 } 00 & 00 & 01 & 02 & 03 & 03 & 04 & 05 & 06 & 07 & 08 \\
10 & 09 & 10 & 10 & 11 & 12 & 13 & 14 & 15 & 16 & 16 \\
20 & 17 & 18 & 19 & 20 & 21 & 22 & 23 & 23 & 24 & 25 \\
30 & 26 & 27 & 28 & 29 & 30 & 30 & 31 & 32 & 33 & 34 \\
40 & 35 & 36 & 36 & 37 & 38 & 39 & 40 & 41 & 42 & 43 \\
50 & 43 & 44 & 45 & 46 & 47 & 48 & 49 & 49 & 50 & 51 \\
60 & 52 & 53 & 54 & 55 & 56 & 56 & 57 & 58 & 59 & 60 \\
70 & 61 & 62 & 63 & 63 & 64 & 65 & 66 & 67 & 68 & 69 \\
80 & 69 & 70 & 71 & 72 & 73 & 74 & 75 & 76 & 76 & 77 \\
90 & 78 & 79 & 80 & 81 & 82 & 82 & 83 & 84 & 85 & 86 \\
100 & 87 & 88 & 89 & 89 & 90 & 91 & 92 & 93 & 94 & 95 \\
110 & 96 & 96 & 97 & 98 & 99 & 100 & 101 & 102 & 102 & 103
\end{tabular}


TABIE 16

Wind Speed

Conversion from kilometers per hour to knots

(1 km/hr $=0.539593 \mathrm{knot}$ )

\begin{tabular}{c|rlllllllll}
\hline $\mathrm{km} / \mathrm{hr}$ & 00 & 01 & 02 & 03 & 04 & 05 & 06 & 07 & 08 & 09 \\
\cline { 2 - 10 } 00 & 00 & 01 & 01 & 02 & 02 & 03 & 03 & 04 & 04 & 05 \\
10 & 05 & 06 & 06 & 07 & 08 & 08 & 09 & 09 & 10 & 10 \\
20 & 11 & 11 & 12 & 12 & 13 & 13 & 14 & 15 & 15 & 16 \\
30 & 16 & 17 & 17 & 18 & 18 & 19 & 19 & 20 & 21 & 21 \\
40 & 22 & 22 & 23 & 23 & 24 & 24 & 25 & 25 & 26 & 26 \\
50 & 27 & 28 & 28 & 29 & 29 & 30 & 30 & 31 & 31 & 32 \\
& & & & & & & & & & \\
60 & 32 & 33 & 33 & 34 & 35 & 35 & 36 & 36 & 37 & 37 \\
70 & 38 & 38 & 39 & 39 & 40 & 40 & 41 & 42 & 42 & 43 \\
80 & 43 & 44 & 44 & 45 & 45 & 46 & 46 & 47 & 47 & 48 \\
90 & 49 & 49 & 50 & 50 & 51 & 51 & 52 & 52 & 53 & 53 \\
100 & 54 & 54 & 55 & 56 & 56 & 57 & 57 & 58 & 58 & 59 \\
110 & 59 & 60 & 60 & 61 & 62 & 62 & 63 & 63 & 64 & 64 \\
120 & 65 & 65 & 66 & 66 & 67 & 67 & 68 & 69 & 69 & 70 \\
130 & 70 & 71 & 71 & 72 & 72 & 73 & 73 & 74 & 74 & 75 \\
140 & 76 & 76 & 77 & 77 & 78 & 78 & 79 & 79 & 80 & 80 \\
150 & 81 & 81 & 82 & 83 & 83 & 84 & 84 & 85 & 85 & 86 \\
160 & 86 & 87 & 87 & 88 & 88 & 89 & 90 & 90 & 91 & 91 \\
170 & 92 & 92 & 93 & 93 & 94 & 94 & 95 & 96 & 96 & 97 \\
180 & 97 & 98 & 98 & 99 & 99 & 100 & 100 & 101 & 101 & 102
\end{tabular}


TABIE 17

Wind Speed

Conversion from feet per second to knots

(1 ftt $/ \mathrm{sec}=0.5921 \mathrm{knot}$ )

\begin{tabular}{c|llllllllll}
\hline $\mathrm{ft} / \mathrm{sec}$ & 00 & 01 & 02 & 03 & 04 & 05 & 06 & 07 & 08 & 09 \\
\cline { 2 - 10 } 00 & 00 & 01 & 01 & 02 & 02 & 03 & 04 & 04 & 05 & 05 \\
10 & 06 & 07 & 07 & 08 & 08 & 09 & 09 & 10 & 11 & 11 \\
20 & 12 & 12 & 13 & 14 & 14 & 15 & 15 & 16 & 17 & 17 \\
30 & 18 & 18 & 19 & 20 & 20 & 21 & 21 & 22 & 22 & 23 \\
40 & 24 & 24 & 25 & 25 & 26 & 27 & 27 & 28 & 28 & 29 \\
50 & 30 & 30 & 31 & 31 & 32 & 33 & 33 & 34 & 34 & 35 \\
60 & 36 & 36 & 37 & 37 & 38 & 38 & 39 & 40 & 40 & 41 \\
70 & 41 & 42 & 43 & 43 & 44 & 44 & 45 & 46 & 46 & 47 \\
80 & 47 & 48 & 49 & 49 & 50 & 50 & 51 & 52 & 52 & 53 \\
90 & 53 & 54 & 54 & 55 & 56 & 56 & 57 & 57 & 58 & 59 \\
100 & 59 & 60 & 60 & 61 & 62 & 62 & 63 & 63 & 64 & 65 \\
110 & 65 & 66 & 66 & 67 & 67 & 68 & 69 & 69 & 70 & 70 \\
120 & 71 & 72 & 72 & 73 & 73 & 74 & 75 & 75 & 76 & 76 \\
130 & 77 & 78 & 78 & 79 & 79 & 80 & 81 & 81 & 82 & 82 \\
140 & 83 & 83 & 84 & 85 & 85 & 86 & 86 & 87 & 88 & 88 \\
150 & 89 & 89 & 90 & 91 & 91 & 92 & 92 & 93 & 94 & 94 \\
160 & 95 & 95 & 96 & 97 & 97 & 98 & 98 & 99 & 99 & 100
\end{tabular}




\section{TABIF 18}

\section{Wind Force}

Conversion from knots, meters per second, kilometers per hour, and miles per hour to the Beaufort wind scale

\begin{tabular}{|c|c|c|c|c|c|}
\hline \multirow{2}{*}{ COOE } & \multirow{2}{*}{ DESCRIPTIVE TERM } & \multicolumn{4}{|c|}{$\begin{array}{l}\text { VELOCITY EOUIVALENT AT A STANDARD HEIGHT } \\
\text { OF } 10 \text { METERS ABOVE OPEN FLAT GROUND }\end{array}$} \\
\hline & & $\begin{array}{l}\text { mean velocity } \\
\text { In knots }\end{array}$ & meters/sec & $\mathrm{km} / \mathrm{h}$ & m.p.h. \\
\hline 0 & Calm & $<1$ & $0-0.2$ & $<1$ & $<1$ \\
\hline 1 & Light air & $1-3$ & $0.3-1.5$ & $1-5$ & $1-3$ \\
\hline 2 & Light breeze & $4-6$ & $1.6-3.3$ & $6-11$ & $4-7$ \\
\hline 3 & Gentle breeze & $7-10$ & $3.4-5.4$ & $12-19$ & $8-12$ \\
\hline 4 & Moderate breeze & $11-16$ & $5.5-7.9$ & $20-28$ & $13-18$ \\
\hline 5 & Fresh breeze & $17-21$ & $8.0-10.7$ & $29-38$ & $19-24$ \\
\hline 6 & Strong breeze & $22-27$ & $10.8-13.8$ & $39-49$ & $25-31$ \\
\hline 7 & Near gale & $28-33$ & $13.9-17.9$ & $50-61$ & $32-38$ \\
\hline 8 & Gale & $34-40$ & $17.2-20.7$ & $62-74$ & $39-46$ \\
\hline 9 & Strong gale & $41-47$ & $20.8-24.4$ & $75-88$ & $47-54$ \\
\hline 10 & Storm & $48-55$ & $24.5-28.4$ & $89-102$ & $55-63$ \\
\hline 11 & Violent storm & $56-63$ & $28.5-32: 6$ & $103-117$ & $64-72$ \\
\hline 12 & Hurricane & $64-71$ & $32.7-36.9$ & $118-133$ & $73-82$ \\
\hline 13 & - & $72-80$ & $37.0-41.4$ & $134-149$ & $83-92$ \\
\hline 14 & - & $81-89$ & $41.5-46.1$ & $150-166$ & $93-103$ \\
\hline 15 & - & $90-99$ & $46.2-50.9$ & $167-183$ & $104-114$ \\
\hline 16 & - & $100-108$ & $51.0-56.0$ & $184-201$ & $115-125$ \\
\hline 17 & - & $109-118$ & $56.1-61.2$ & $202-220$ & $126-136$ \\
\hline
\end{tabular}


TABLE 19

Atmospheric Pressure

Conversion from inches of mercury to millibars* (I inch of $\mathrm{H}_{\mathrm{g}}=33.8639 \mathrm{mbs}$ )

\begin{tabular}{|c|c|c|c|c|c|c|c|c|c|c|}
\hline Inches & .00 & .01 & .02 & .03 & .04 & .05 & .06 & .07 & .08 & .09 \\
\hline $\begin{array}{l}27.9 \\
28.0\end{array}$ & $\begin{array}{l}44.8 \\
48.2\end{array}$ & $\begin{array}{l}45.1 \\
48.5\end{array}$ & $\begin{array}{l}45.5 \\
48.9\end{array}$ & $\begin{array}{l}45.8 \\
49.2\end{array}$ & $\begin{array}{l}46.2 \\
49.5\end{array}$ & $\begin{array}{l}46.5 \\
49.9\end{array}$ & $\begin{array}{l}46.8 \\
50.2\end{array}$ & $\begin{array}{l}47.2 \\
50.6\end{array}$ & $\begin{array}{l}47.5 \\
50.9\end{array}$ & $\begin{array}{l}47.9 \\
51.2\end{array}$ \\
\hline $\begin{array}{l}28.1 \\
28.2 \\
28.3 \\
28.4 \\
28.5\end{array}$ & $\begin{array}{l}51.6 \\
55.0 \\
58.3 \\
61.7 \\
65.1\end{array}$ & $\begin{array}{l}51.9 \\
55.3 \\
58.7 \\
62.1 \\
65.5\end{array}$ & $\begin{array}{l}52.3 \\
55.6 \\
59.0 \\
62.4 \\
65.8\end{array}$ & $\begin{array}{l}52.6 \\
56.0 \\
59.4 \\
62.8 \\
66.1\end{array}$ & $\begin{array}{l}52.9 \\
56.3 \\
59.7 \\
63.1 \\
66.5\end{array}$ & $\begin{array}{l}53.3 \\
56.7 \\
60.0 \\
63.4 \\
66.8\end{array}$ & $\begin{array}{l}53.6 \\
57.0 \\
60.4 \\
63.8 \\
67.2\end{array}$ & $\begin{array}{l}53 \cdot 9 \\
57 \cdot 3 \\
60.7 \\
64.1 \\
67 \cdot 5\end{array}$ & $\begin{array}{l}54.3 \\
57.7 \\
61.1 \\
64.4 \\
67.8\end{array}$ & $\begin{array}{l}54.6 \\
58.0 \\
61.4 \\
64.8 \\
68.2\end{array}$ \\
\hline $\begin{array}{l}28.6 \\
28.7 \\
28.8 \\
28.9 \\
29.0\end{array}$ & $\begin{array}{l}68.5 \\
71.9 \\
75.3 \\
78.7 \\
82.1\end{array}$ & $\begin{array}{l}68.8 \\
72.2 \\
75.6 \\
79.0 \\
82.4\end{array}$ & $\begin{array}{l}69.2 \\
72.6 \\
76.0 \\
79.3 \\
82.7\end{array}$ & $\begin{array}{l}69.5 \\
72.9 \\
76.3 \\
79.7 \\
83.1\end{array}$ & $\begin{array}{l}69.9 \\
73.2 \\
76.6 \\
80.0 \\
83.4\end{array}$ & $\begin{array}{l}70.2 \\
73.6 \\
77.0 \\
80.4 \\
83.7\end{array}$ & $\begin{array}{l}70.5 \\
73.9 \\
77.3 \\
80.7 \\
84.1\end{array}$ & $\begin{array}{l}70.9 \\
74.3 \\
77.7 \\
81.0 \\
84.4\end{array}$ & $\begin{array}{l}71.2 \\
74.6 \\
78.0 \\
81.4 \\
84.8\end{array}$ & $\begin{array}{l}71.6 \\
74.9 \\
78.3 \\
81.7 \\
85.1\end{array}$ \\
\hline $\begin{array}{l}29 \cdot 1 \\
29 \cdot 2 \\
29 \cdot 3 \\
29 \cdot 4 \\
29 \cdot 5\end{array}$ & $\begin{array}{l}85.4 \\
88.8 \\
92.2 \\
95.6 \\
99.0\end{array}$ & $\begin{array}{l}85.8 \\
89.2 \\
92.6 \\
95.9 \\
99.3\end{array}$ & $\begin{array}{l}86.1 \\
89.5 \\
92.9 \\
96.3 \\
99.7\end{array}$ & $\begin{array}{l}86.5 \\
89.8 \\
93.2 \\
96.6 \\
00.0\end{array}$ & $\begin{array}{l}86.8 \\
90.2 \\
93.6 \\
97.0 \\
00.3\end{array}$ & $\begin{array}{l}87.1 \\
90.5 \\
93 \cdot 9 \\
97 \cdot 3 \\
00.7\end{array}$ & $\begin{array}{l}87.5 \\
90.9 \\
94.2 \\
97.6 \\
01.0\end{array}$ & $\begin{array}{l}87.8 \\
91.2 \\
94.6 \\
98.0 \\
01.4\end{array}$ & $\begin{array}{l}88.1 \\
91.5 \\
94.9 \\
98.3 \\
01.7\end{array}$ & $\begin{array}{l}88.5 \\
91.9 \\
95.3 \\
98.6 \\
02.0\end{array}$ \\
\hline $\begin{array}{l}29.6 \\
29.7 \\
29.8 \\
29.9 \\
30.0\end{array}$ & $\begin{array}{l}02.4 \\
05.8 \\
09.1 \\
12.5 \\
15.9\end{array}$ & $\begin{array}{l}02.7 \\
06.1 \\
09.5 \\
12.9 \\
16.3\end{array}$ & $\begin{array}{l}03.0 \\
06.4 \\
09.8 \\
13.2 \\
16.6\end{array}$ & $\begin{array}{l}03.4 \\
06.8 \\
10.2 \\
13.5 \\
16.9\end{array}$ & $\begin{array}{l}03.7 \\
07.1 \\
10.5 \\
13.9 \\
17.3\end{array}$ & $\begin{array}{l}04.1 \\
07.5 \\
10.8 \\
14.2 \\
17.6\end{array}$ & $\begin{array}{l}04.4 \\
07.8 \\
11.2 \\
14.6 \\
17.9\end{array}$ & $\begin{array}{l}04.7 \\
08.1 \\
11.5 \\
14.9 \\
18.3\end{array}$ & $\begin{array}{l}05.1 \\
08.5 \\
11.9 \\
15.2 \\
18.6\end{array}$ & $\begin{array}{l}05.4 \\
08.8 \\
12.2 \\
15.6 \\
19.0\end{array}$ \\
\hline $\begin{array}{l}30.1 \\
30.2 \\
30.3 \\
30.4 \\
30.5\end{array}$ & $\begin{array}{l}19.3 \\
22.7 \\
26.1 \\
29.5 \\
32.8\end{array}$ & $\begin{array}{l}19.6 \\
23.0 \\
26.4 \\
29.8 \\
33.2\end{array}$ & $\begin{array}{l}20.0 \\
23.4 \\
26.8 \\
30.1 \\
33.5\end{array}$ & $\begin{array}{l}20.3 \\
23.7 \\
27.1 \\
30.5 \\
33.9\end{array}$ & $\begin{array}{l}20.7 \\
24.0 \\
27.4 \\
30.8 \\
34.2\end{array}$ & $\begin{array}{l}21.0 \\
24.4 \\
27.8 \\
31.2 \\
34.5\end{array}$ & $\begin{array}{l}21.3 \\
24.7 \\
28.1 \\
31.5 \\
34.9\end{array}$ & $\begin{array}{l}21.7 \\
25.1 \\
28.4 \\
31.8 \\
35.2\end{array}$ & $\begin{array}{l}22.0 \\
25.4 \\
28.8 \\
32.2 \\
35.6\end{array}$ & $\begin{array}{l}22.4 \\
25.7 \\
29.1 \\
32.5 \\
35.9\end{array}$ \\
\hline $\begin{array}{l}30.6 \\
30.7 \\
30.8 \\
30.9 \\
31.0\end{array}$ & $\begin{array}{l}36.2 \\
39.6 \\
43.0 \\
46.4 \\
49.8\end{array}$ & $\begin{array}{l}36.6 \\
40.0 \\
43.3 \\
46.7 \\
50.1\end{array}$ & $\begin{array}{l}36.9 \\
40.3 \\
43.7 \\
47.1 \\
50.5\end{array}$ & $\begin{array}{l}37.3 \\
40.6 \\
44.0 \\
47.4 \\
50.8\end{array}$ & $\begin{array}{l}37.6 \\
41.0 \\
44.4 \\
47.7 \\
51.1\end{array}$ & $\begin{array}{l}37.9 \\
41.3 \\
44.7 \\
48.1 \\
51.5\end{array}$ & $\begin{array}{l}38.3 \\
41.7 \\
45.0 \\
48.4 \\
51.8\end{array}$ & $\begin{array}{l}38.6 \\
42.0 \\
45.4 \\
48.8 \\
52.2\end{array}$ & $\begin{array}{l}38.9 \\
42.3 \\
45.7 \\
49.1 \\
52.5\end{array}$ & $\begin{array}{l}39.3 \\
42.7 \\
46.1 \\
49.4 \\
52.8\end{array}$ \\
\hline
\end{tabular}

*The hundreds and thousands digits are not recorded; the true range of this table is $944.8-1052.8 \mathrm{mbs}$. 
Atmospheric Pressure

Conversion from millimeters of mercury to millibars*

( $1 \mathrm{~mm}$ of $\mathrm{Hg}=1.33322 \mathrm{mbs}$ )

\begin{tabular}{|c|c|c|c|c|c|c|c|c|c|c|}
\hline $\mathrm{mm}$ of $\mathrm{Hg}$ & 0.0 & 0.1 & 0.2 & 0.3 & 0.4 & 0.5 & 0.6 & 0.7 & 0.8 & 0.9 \\
\hline $\begin{array}{l}708 \\
709 \\
710\end{array}$ & $\begin{array}{l}43.9 \\
45.3 \\
46.6\end{array}$ & $\begin{array}{l}44.1 \\
45.4 \\
46.7\end{array}$ & $\begin{array}{l}44.2 \\
45.5 \\
46.9\end{array}$ & $\begin{array}{l}44.3 \\
45.7 \\
47.0\end{array}$ & $\begin{array}{l}44.5 \\
45.8 \\
47.1\end{array}$ & $\begin{array}{l}44.6 \\
45.9 \\
47.3\end{array}$ & $\begin{array}{l}44.7 \\
46.7 \\
47.4\end{array}$ & $\begin{array}{l}44.9 \\
46.2 \\
47.5\end{array}$ & $\begin{array}{l}45.0 \\
46.3 \\
47.7\end{array}$ & $\begin{array}{l}45.1 \\
46.5 \\
47.8\end{array}$ \\
\hline $\begin{array}{l}711 \\
712 \\
713 \\
714 \\
715\end{array}$ & $\begin{array}{l}47.9 \\
49.3 \\
50.6 \\
51.9 \\
53.3\end{array}$ & $\begin{array}{l}48.1 \\
49.4 \\
50.7 \\
52.1 \\
53.4\end{array}$ & $\begin{array}{l}48.2 \\
49.5 \\
50.9 \\
52.2 \\
53.5\end{array}$ & $\begin{array}{l}48.3 \\
49.7 \\
51.0 \\
52.3 \\
53.7\end{array}$ & $\begin{array}{l}48.5 \\
49.8 \\
51.1 \\
52.5 \\
53.8\end{array}$ & $\begin{array}{l}48.6 \\
49.9 \\
51.3 \\
52.6 \\
53.9\end{array}$ & $\begin{array}{l}48.7 \\
50.1 \\
51.4 \\
52.7 \\
54.1\end{array}$ & $\begin{array}{l}48.9 \\
50.2 \\
51.5 \\
52.9 \\
54.2\end{array}$ & $\begin{array}{l}49.0 \\
50.3 \\
51.7 \\
53.0 \\
54.3\end{array}$ & $\begin{array}{l}49.1 \\
50.5 \\
51.8 \\
53.1 \\
54.5\end{array}$ \\
\hline $\begin{array}{l}716 \\
717 \\
718 \\
719 \\
720\end{array}$ & $\begin{array}{l}54.6 \\
55.9 \\
57.3 \\
58.6 \\
59.9\end{array}$ & $\begin{array}{l}54.7 \\
56.1 \\
57.4 \\
58.7 \\
60.1\end{array}$ & $\begin{array}{l}54.9 \\
56.2 \\
57.5 \\
58.9 \\
60.2\end{array}$ & $\begin{array}{l}55.0 \\
56.3 \\
57.7 \\
59.0 \\
60.3\end{array}$ & $\begin{array}{l}55.1 \\
56.5 \\
57.8 \\
59.1 \\
60.5\end{array}$ & $\begin{array}{l}55.3 \\
56.6 \\
57.9 \\
59.3 \\
60.6\end{array}$ & $\begin{array}{l}55.4 \\
56.7 \\
58.1 \\
59.4 \\
60.7\end{array}$ & $\begin{array}{l}55.5 \\
56.9 \\
58.2 \\
59.5 \\
60.9\end{array}$ & $\begin{array}{l}55.7 \\
57.0 \\
58.3 \\
59.7 \\
61.0\end{array}$ & $\begin{array}{l}55.8 \\
57.1 \\
58.5 \\
59.8 \\
61.1\end{array}$ \\
\hline $\begin{array}{l}721 \\
722 \\
723 \\
724 \\
725\end{array}$ & $\begin{array}{l}61.3 \\
62.6 \\
63.9 \\
65.3 \\
66.6\end{array}$ & $\begin{array}{l}61.4 \\
62.7 \\
64.1 \\
65.4 \\
66.7\end{array}$ & $\begin{array}{l}61.5 \\
62.9 \\
64.2 \\
65.5 \\
66.9\end{array}$ & $\begin{array}{l}61.7 \\
63.0 \\
64.3 \\
65.7 \\
67.0\end{array}$ & $\begin{array}{l}61.8 \\
63.1 \\
64.5 \\
65.8 \\
67.1\end{array}$ & $\begin{array}{l}61.9 \\
63.3 \\
64.6 \\
65.9 \\
67.3\end{array}$ & $\begin{array}{l}62.1 \\
63.4 \\
64.7 \\
66.1 \\
67.4\end{array}$ & $\begin{array}{l}62.2 \\
63.5 \\
64.9 \\
66.2 \\
67.5\end{array}$ & $\begin{array}{l}62.3 \\
63.7 \\
65.0 \\
66.3 \\
67.7\end{array}$ & $\begin{array}{l}62.5 \\
63.8 \\
65.1 \\
66.5 \\
67.8\end{array}$ \\
\hline $\begin{array}{l}726 \\
727 \\
728 \\
729 \\
730\end{array}$ & $\begin{array}{l}67.9 \\
69.3 \\
70.6 \\
71.9 \\
73.3\end{array}$ & $\begin{array}{l}68.1 \\
69.4 \\
70.7 \\
72.1 \\
73.4\end{array}$ & $\begin{array}{l}68.2 \\
69.5 \\
70.9 \\
72.2 \\
73.5\end{array}$ & $\begin{array}{l}68.3 \\
69.7 \\
71.0 \\
72.3 \\
73.7\end{array}$ & $\begin{array}{l}68.5 \\
69.8 \\
71.1 \\
72.5 \\
73.8\end{array}$ & $\begin{array}{l}68.6 \\
69.9 \\
71.3 \\
72.6 \\
73.9\end{array}$ & $\begin{array}{l}68.7 \\
70.1 \\
71.4 \\
72.7 \\
74.1\end{array}$ & $\begin{array}{l}68.9 \\
70.2 \\
71.5 \\
72.9 \\
74.2\end{array}$ & $\begin{array}{l}69.0 \\
70.3 \\
71.7 \\
73.0 \\
74.3\end{array}$ & $\begin{array}{l}69.1 \\
70.5 \\
71.8 \\
73.1 \\
74.5\end{array}$ \\
\hline $\begin{array}{l}731 \\
732 \\
733 \\
734 \\
735\end{array}$ & $\begin{array}{l}74.6 \\
75.9 \\
77.3 \\
78.6 \\
79.9\end{array}$ & $\begin{array}{l}74.7 \\
76.1 \\
77.4 \\
78.7 \\
80.1\end{array}$ & $\begin{array}{l}74.9 \\
76.2 \\
77.5 \\
78.9 \\
80.2\end{array}$ & $\begin{array}{l}75.0 \\
76.3 \\
77.7 \\
79.0 \\
80.3\end{array}$ & $\begin{array}{l}75.1 \\
76.5 \\
77.8 \\
79.1 \\
80.5\end{array}$ & $\begin{array}{l}75.3 \\
76.6 \\
77.9 \\
79.3 \\
80.6\end{array}$ & $\begin{array}{l}75.4 \\
76.7 \\
78.1 \\
79.4 \\
80.7\end{array}$ & $\begin{array}{l}75.5 \\
76.9 \\
78.2 \\
79.5 \\
80.9\end{array}$ & $\begin{array}{l}75.7 \\
77.0 \\
78.3 \\
79.7 \\
81.0\end{array}$ & $\begin{array}{l}75.8 \\
77.1 \\
78.5 \\
79.8 \\
81.1\end{array}$ \\
\hline
\end{tabular}

*The hundreds digit is not recorded. The true range of this part of Table 20 is $943.9 \mathrm{mbs}-981.1 \mathrm{mbs}$. 
TABLE 20 (Cont'd)

Atmospheric Pressure

Conversion from millimeters of mercury to millibars* (Cont'd)

( $1 \mathrm{~mm}$ of $\mathrm{Hg}=1.33322 \mathrm{mbs}$ )

\begin{tabular}{|c|c|c|c|c|c|c|c|c|c|c|}
\hline $\mathrm{mm}$ of $\mathrm{Hg}$ & 0.0 & 0.1 & 0.2 & 0.3 & 0.4 & 0.5 & 0.6 & 0.7 & 0.8 & 0.9 \\
\hline $\begin{array}{l}736 \\
737 \\
738 \\
739 \\
740\end{array}$ & $\begin{array}{l}81.3 \\
82.6 \\
83.9 \\
85.3 \\
86.6\end{array}$ & $\begin{array}{l}81.4 \\
82.7 \\
84.1 \\
85.4 \\
86.7\end{array}$ & $\begin{array}{l}81.5 \\
82.9 \\
84.2 \\
85.5 \\
86.9\end{array}$ & $\begin{array}{l}81.7 \\
83.0 \\
84.3 \\
85.7 \\
87.0\end{array}$ & $\begin{array}{l}81.8 \\
83.1 \\
84.5 \\
85.8 \\
87.1\end{array}$ & $\begin{array}{l}81.9 \\
83 \cdot 3 \\
84.6 \\
85.9 \\
87 \cdot 3\end{array}$ & $\begin{array}{l}82.1 \\
83.4 \\
84.7 \\
86.1 \\
87.4\end{array}$ & $\begin{array}{l}82.2 \\
83.5 \\
84.9 \\
86.2 \\
87.5\end{array}$ & $\begin{array}{l}82.3 \\
83.7 \\
85.0 \\
86.3 \\
87.7\end{array}$ & $\begin{array}{l}82.5 \\
83.8 \\
85.1 \\
86.5 \\
87.8\end{array}$ \\
\hline $\begin{array}{l}741 \\
742 \\
743 \\
744 \\
745\end{array}$ & $\begin{array}{l}87 \cdot 9 \\
89 \cdot 3 \\
90.6 \\
91 \cdot 9 \\
93 \cdot 3\end{array}$ & $\begin{array}{l}88.1 \\
89.4 \\
90.7 \\
92.1 \\
93.4\end{array}$ & $\begin{array}{l}88.2 \\
89.5 \\
90.9 \\
92.2 \\
93.5\end{array}$ & $\begin{array}{l}88.3 \\
89.7 \\
91.0 \\
92.3 \\
93.7\end{array}$ & $\begin{array}{l}88.5 \\
89.8 \\
91.1 \\
92.5 \\
93.8\end{array}$ & $\begin{array}{l}88.6 \\
89.9 \\
91.3 \\
92.6 \\
93.9\end{array}$ & $\begin{array}{l}88.7 \\
90.1 \\
91.4 \\
92.7 \\
94.1\end{array}$ & $\begin{array}{l}88.9 \\
90.2 \\
91.5 \\
92.9 \\
94.2\end{array}$ & $\begin{array}{l}89.0 \\
90.3 \\
91.7 \\
93.0 \\
94.3\end{array}$ & $\begin{array}{l}89.1 \\
90.5 \\
91.8 \\
93.1 \\
94.5\end{array}$ \\
\hline $\begin{array}{l}746 \\
747 \\
748 \\
749 \\
750\end{array}$ & $\begin{array}{l}94.6 \\
95.9 \\
97.3 \\
98.6 \\
99.9\end{array}$ & $\begin{array}{l}94.7 \\
96.1 \\
97.4 \\
98.7 \\
00.1\end{array}$ & $\begin{array}{l}94.9 \\
96.2 \\
97.5 \\
98.9 \\
00.2\end{array}$ & $\begin{array}{l}95.0 \\
96.3 \\
97.7 \\
99.0 \\
00.3\end{array}$ & $\begin{array}{l}95.1 \\
96.5 \\
97.8 \\
99.1 \\
00.5\end{array}$ & $\begin{array}{l}95.3 \\
96.6 \\
97.9 \\
99.3 \\
00.6\end{array}$ & $\begin{array}{l}95.4 \\
96.7 \\
98.1 \\
99.4 \\
00.7\end{array}$ & $\begin{array}{l}95.5 \\
96.9 \\
98.2 \\
99.5 \\
00.9\end{array}$ & $\begin{array}{l}95.7 \\
97.0 \\
98.3 \\
99.7 \\
01.0\end{array}$ & $\begin{array}{l}95.8 \\
97.1 \\
98.5 \\
99.8 \\
01.1\end{array}$ \\
\hline $\begin{array}{l}751 \\
752 \\
753 \\
754 \\
755\end{array}$ & $\begin{array}{l}01.3 \\
02.6 \\
03.9 \\
05.3 \\
06.6\end{array}$ & $\begin{array}{l}01.4 \\
02.7 \\
04.1 \\
05.4 \\
06.7\end{array}$ & $\begin{array}{l}01.5 \\
02.9 \\
04.2 \\
05.5 \\
06.9\end{array}$ & $\begin{array}{l}01.7 \\
03.0 \\
04.3 \\
05.7 \\
07.0\end{array}$ & $\begin{array}{l}01.8 \\
03.1 \\
04.5 \\
05.8 \\
07.1\end{array}$ & $\begin{array}{l}01.9 \\
03.3 \\
04.6 \\
05.9 \\
07.3\end{array}$ & $\begin{array}{l}02.1 \\
03.4 \\
04.7 \\
06.1 \\
07.4\end{array}$ & $\begin{array}{l}02.2 \\
03.5 \\
04.9 \\
06.2 \\
07.5\end{array}$ & $\begin{array}{l}02.3 \\
03.7 \\
05.0 \\
06.3 \\
07.7\end{array}$ & $\begin{array}{l}02.5 \\
03.8 \\
05.1 \\
06.5 \\
07.8\end{array}$ \\
\hline $\begin{array}{l}756 \\
757 \\
758 \\
759 \\
760\end{array}$ & $\begin{array}{l}07.9 \\
09.3 \\
10.6 \\
11.9 \\
13.3\end{array}$ & $\begin{array}{l}08.1 \\
09.4 \\
10.7 \\
12.1 \\
13.4\end{array}$ & $\begin{array}{l}08.2 \\
09.5 \\
10.9 \\
12.2 \\
13.5\end{array}$ & $\begin{array}{l}08.3 \\
09.7 \\
11.0 \\
12.3 \\
13.7\end{array}$ & $\begin{array}{l}08.5 \\
09.8 \\
11.1 \\
12.5 \\
13.8\end{array}$ & $\begin{array}{l}08.6 \\
09.9 \\
11.3 \\
12.6 \\
13.9\end{array}$ & $\begin{array}{l}08.7 \\
10.1 \\
11.4 \\
12.7 \\
14.1\end{array}$ & $\begin{array}{l}08.9 \\
10.2 \\
11.5 \\
12.9 \\
14.2\end{array}$ & $\begin{array}{l}09.0 \\
10.3 \\
11.7 \\
13.0 \\
14.3\end{array}$ & $\begin{array}{l}09.1 \\
10.5 \\
11.8 \\
13.1 \\
14.5\end{array}$ \\
\hline $\begin{array}{l}761 \\
762 \\
763 \\
764 \\
765\end{array}$ & $\begin{array}{l}14.6 \\
15.9 \\
17.2 \\
18.6 \\
19.9\end{array}$ & $\begin{array}{l}14.7 \\
16.1 \\
17.4 \\
18.7 \\
20.0\end{array}$ & $\begin{array}{l}14.9 \\
16.2 \\
17.5 \\
18.8 \\
20.2\end{array}$ & $\begin{array}{l}15.0 \\
16.3 \\
17.6 \\
19.0 \\
20.3\end{array}$ & $\begin{array}{l}15.1 \\
16.4 \\
17.8 \\
19.1 \\
20.4\end{array}$ & $\begin{array}{l}15.3 \\
16.6 \\
17.9 \\
19.2 \\
20.6\end{array}$ & $\begin{array}{l}15.4 \\
16.7 \\
18.0 \\
19.4 \\
20.7\end{array}$ & $\begin{array}{l}15.5 \\
16.8 \\
18.2 \\
19.5 \\
20.8\end{array}$ & $\begin{array}{l}15.7 \\
17.0 \\
18.3 \\
19.6 \\
21.0\end{array}$ & $\begin{array}{l}15.8 \\
17.1 \\
18.4 \\
19.8 \\
21.1\end{array}$ \\
\hline
\end{tabular}

*The hundreds and thousands digits are not recorded. The true range of this part of Table 20 is $981.3 \mathrm{mbs}$ - $1021.1 \mathrm{mbs}$. 
TABIE 20 (Cont'd)

Atmospheric Pressure

Conversion from millimeters of mercury to millibars* (Cont'd)

( $1 \mathrm{~mm}$ of $\mathrm{Hg}=1.33322 \mathrm{mbs}$ )

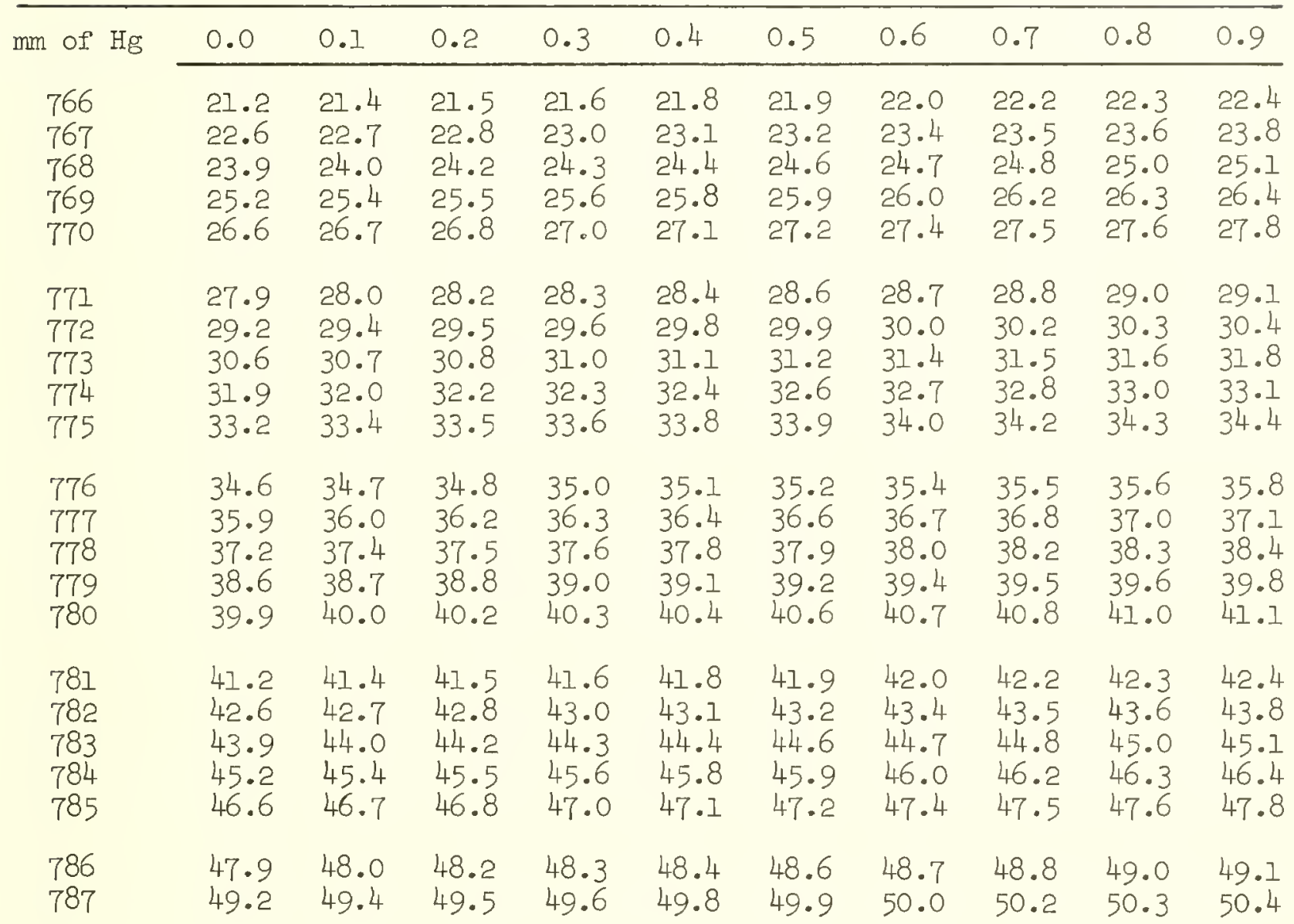

*The hundreds and thousands digits are not recorded. The true range of this part of Table 20 is $1021.2 \mathrm{mbs}$ - $1050.4 \mathrm{mbs}$. 
Conversion from Fahrenheit to Centigrade

\begin{tabular}{|c|c|c|c|c|c|c|c|c|c|c|}
\hline${ }^{\circ} \mathrm{F}$ & 0.0 & 0.1 & 0.2 & 0.3 & 0.4 & 0.5 & 0.6 & 0.7 & 0.8 & 0.9 \\
\hline 130 & 54.4 & 54.5 & 54.6 & 54.6 & 54.7 & 54.7 & 54.8 & 54.8 & 54.9 & 54.9 \\
\hline 129 & 53.9 & 53.9 & 54.0 & 54.1 & 54.1 & 54.2 & 54.2 & 54.3 & 54.3 & 54.4 \\
\hline 128 & 53.3 & 53.4 & 53.5 & 53.6 & 53.6 & 53.6 & 53.7 & 53.7 & 53.8 & 53.8 \\
\hline 127 & 52.8 & 52.8 & 52.9 & 52.9 & 53.0 & 53.1 & 53.1 & 53.2 & 53.2 & 53.3 \\
\hline 126 & 52.2 & 52.3 & $52 \cdot 3$ & 52.4 & 52.4 & 52.5 & 52.6 & 52.6 & 52.7 & 52.7 \\
\hline 125 & 51.7 & 51.7 & 51.8 & 51.8 & 51.9 & 51.9 & 52.0 & 52.1 & 52.1 & 52.2 \\
\hline 124 & 51.1 & 51.2 & 51.2 & 51.3 & 51.3 & 51.4 & 51.4 & 51.5 & 51.6 & 51.6 \\
\hline 123 & 50.6 & 50.6 & 50.7 & 50.7 & 50.8 & 50.8 & 50.9 & 50.9 & 51.0 & 51.1 \\
\hline 122 & 50.0 & 50.1 & 50.1 & 50.2 & 50.2 & 50.3 & 50.3 & 50.4 & 50.4 & 50.5 \\
\hline 121 & 49.4 & 49.5 & 49.6 & 49.6 & 49.7 & 49.7 & 49.8 & 49.8 & 49.9 & 49.9 \\
\hline 120 & 48.9 & 48.9 & 49.0 & 49.1 & 49.1 & 49.2 & 49.2 & 49.3 & 49.3 & 49.4 \\
\hline 119 & 48.3 & 48.4 & 48.4 & 48.5 & 48.6 & 48.6 & 48.7 & 48.7 & 48.8 & 48.8 \\
\hline 118 & 47.8 & 47.8 & 47.9 & 47.9 & 48.0 & 48.1 & 48.1 & 48.2 & 48.2 & 48.3 \\
\hline 117 & 47.2 & 47.3 & 47.3 & 47.4 & 47.4 & 47.5 & 47.6 & 47.6 & 47.7 & 47.7 \\
\hline 116 & 46.7 & 46.7 & 46.8 & 46.8 & 46.9 & 46.9 & 47.0 & 47.1 & 47.1 & 47.2 \\
\hline 115 & 46.1 & 46.2 & 46.2 & 46.3 & 46.3 & 46.4 & 46.4 & 46.5 & 46.6 & 46.6 \\
\hline 114 & 45.6 & 45.6 & 45.7 & 45.7 & 45.8 & 45.8 & 45.9 & 45.9 & 46.0 & 46.1 \\
\hline 113 & 45.0 & 45.1 & 45.1 & 45.2 & 45.2 & 45.3 & 45.3 & 45.4 & 45.4 & 45.5 \\
\hline 112 & 44.4 & 44.5 & 44.6 & 44.7 & 44.7 & 44.7 & 44.8 & 44.8 & 44.9 & 44.9 \\
\hline 111 & 43.9 & 43.9 & 44.0 & 44.1 & 44.1 & 44.2 & 44.2 & 44.3 & 44.3 & 44.4 \\
\hline 110 & 43.3 & 43.4 & 43.4 & 43.5 & 43.6 & 43.6 & 43.7 & 43.7 & 43.8 & 43.8 \\
\hline 109 & 42.8 & 42.8 & 42.9 & 43.9 & 43.0 & 43.1 & 43.1 & 43.2 & 43.2 & 43.3 \\
\hline 108 & 42.2 & 42.3 & 42.3 & 42.4 & 42.4 & 42.5 & 42.6 & 42.6 & 42.7 & 42.7 \\
\hline 107 & 41.7 & 41.7 & 41.8 & 41.8 & 41.9 & 41.9 & 42.0 & 42.1 & 42.1 & 42.2 \\
\hline 106 & 41.1 & 41.2 & 41.2 & 41.3 & 41.3 & 41.4 & 41.4 & 41.5 & 41.6 & 41.6 \\
\hline 105 & 40.6 & 40.6 & 40.7 & 40.7 & 40.8 & 40.8 & 40.9 & 40.9 & 41.0 & 41.1 \\
\hline 104 & 40.0 & 40.1 & 40.1 & 40.2 & 40.2 & 40.3 & 40.3 & 40.4 & 40.4 & 40.5 \\
\hline
\end{tabular}




\section{TABIE $2 l$ (Cont'd)}

\section{Temperature}

Conversion from Fahrenheit to Centigrade

\begin{tabular}{c|cccccccccc}
\hline$\circ$ & \multicolumn{1}{c}{0.0} & 0.1 & 0.2 & 0.3 & 0.4 & 0.5 & 0.6 & 0.7 & 0.8 & 0.9 \\
\cline { 2 - 10 } 103 & 39.4 & 39.5 & 39.6 & 39.6 & 39.7 & 39.7 & 39.8 & 39.8 & 39.9 & 39.9 \\
102 & 38.9 & 38.9 & 39.0 & 39.1 & 39.1 & 39.2 & 39.2 & 39.3 & 39.3 & 39.4 \\
101 & 38.3 & 38.4 & 38.4 & 38.5 & 38.6 & 38.6 & 38.7 & 38.7 & 38.8 & 38.8 \\
100 & 37.8 & 37.8 & 37.9 & 37.9 & 38.0 & 38.1 & 38.1 & 38.2 & 38.2 & 38.3 \\
99 & 37.2 & 37.3 & 37.3 & 37.4 & 37.4 & 37.5 & 37.6 & 37.6 & 37.7 & 37.7 \\
98 & 36.7 & 36.7 & 36.8 & 36.8 & 36.9 & 36.9 & 37.0 & 37.1 & 37.1 & 37.2 \\
97 & 36.1 & 36.2 & 36.2 & 36.2 & 36.3 & 36.4 & 36.4 & 36.5 & 36.6 & 36.6 \\
96 & 35.6 & 35.6 & 35.7 & 35.7 & 35.8 & 35.8 & 35.9 & 35.9 & 36.0 & 36.1 \\
95 & 35.0 & 35.1 & 35.1 & 35.2 & 35.2 & 35.3 & 35.3 & 35.4 & 35.4 & 35.5 \\
94 & 34.4 & 34.5 & 34.6 & 34.6 & 34.7 & 34.7 & 34.8 & 34.8 & 34.9 & 34.9 \\
93 & 33.9 & 33.9 & 34.0 & 34.1 & 34.1 & 34.2 & 34.2 & 34.3 & 34.3 & 34.4 \\
92 & 33.3 & 33.4 & 33.4 & 33.5 & 33.6 & 33.6 & 33.7 & 33.7 & 33.8 & 33.8 \\
91 & 32.8 & 32.8 & 32.9 & 32.9 & 33.0 & 33.1 & 33.1 & 33.2 & 33.2 & 33.3 \\
90 & 32.2 & 32.3 & 32.3 & 32.4 & 32.4 & 32.5 & 32.6 & 32.6 & 32.7 & 32.7 \\
89 & 31.7 & 31.7 & 31.8 & 31.8 & 31.9 & 31.9 & 32.0 & 32.1 & 32.1 & 32.2 \\
88 & 31.1 & 31.2 & 31.2 & 31.3 & 31.3 & 31.4 & 31.4 & 31.5 & 31.6 & 31.6 \\
87 & 30.6 & 30.6 & 30.7 & 30.7 & 30.8 & 30.8 & 30.9 & 30.9 & 31.0 & 31.1 \\
86 & 30.0 & 30.1 & 30.1 & 30.2 & 30.2 & 30.3 & 30.3 & 30.4 & 30.4 & 30.5 \\
85 & 29.4 & 29.5 & 29.6 & 29.6 & 29.7 & 29.7 & 29.8 & 29.8 & 29.9 & 29.9 \\
84 & 28.9 & 28.9 & 29.0 & 29.1 & 29.1 & 29.2 & 29.2 & 29.3 & 29.3 & 29.3 \\
83 & 28.3 & 28.4 & 28.4 & 28.5 & 28.6 & 28.6 & 28.7 & 28.7 & 28.8 & 28.8 \\
82 & 27.8 & 27.8 & 27.9 & 28.9 & 28.0 & 28.1 & 28.1 & 28.2 & 28.2 & 28.3 \\
81 & 27.2 & 27.3 & 27.3 & 27.4 & 27.4 & 27.5 & 27.6 & 27.6 & 27.7 & 27.7 \\
80 & 26.7 & 26.7 & 26.8 & 26.8 & 26.9 & 26.9 & 27.0 & 27.1 & 27.1 & 27.2 \\
79 & 26.1 & 26.2 & 26.2 & 26.3 & 26.3 & 26.4 & 26.4 & 26.5 & 26.6 & 26.6 \\
78 & 25.6 & 25.6 & 25.7 & 25.7 & 25.8 & 25.8 & 25.9 & 25.9 & 26.0 & 26.1 \\
77 & 25.0 & 25.1 & 25.1 & 25.2 & 25.2 & 25.3 & 25.3 & 25.4 & 25.4 & 25.5 \\
& & & & & & & & & & \\
\hline 6
\end{tabular}


TABIE 21 (Cont'd)

Temperature

Conversion from Fahrenheit to Centigrade

\begin{tabular}{|c|c|c|c|c|c|c|c|c|c|c|}
\hline${ }^{\circ} \mathrm{F}$ & 0.0 & 0.1 & 0.2 & 0.3 & 0.4 & 0.5 & 0.6 & 0.7 & 0.8 & 0.9 \\
\hline 76 & 24.4 & 24.5 & 24.6 & 24.6 & 24.7 & 24.7 & 24.8 & 24.8 & 24.9 & 24.9 \\
\hline 75 & 23.9 & 23.9 & 24.0 & 24.1 & 24.1 & 24.2 & 24.2 & 24.3 & $24 \cdot 3$ & 24.4 \\
\hline 74 & 23.3 & 23.4 & 23.4 & 23.5 & 23.6 & 23.6 & 23.7 & 23.7 & 23.8 & 23.8 \\
\hline 73 & 22.8 & 22.8 & 22.9 & 22.9 & 23.0 & 23.1 & 23.1 & 23.2 & 23.2 & 23.3 \\
\hline 72 & 22.2 & $22 \cdot 3$ & $22 \cdot 3$ & 22.4 & 22.4 & 22.5 & 22.6 & 22.6 & 22.7 & 22.7 \\
\hline 71 & 21.7 & 21.7 & 21.8 & 21.8 & 21.9 & 21.9 & 22.0 & 22.1 & 22.1 & 22.2 \\
\hline 70 & 21.1 & 21.2 & 21.2 & 21.3 & 21.3 & 21.4 & 21.4 & 21.5 & 21.6 & 21.6 \\
\hline 69 & 20.6 & 20.6 & 20.7 & 20.7 & 20.8 & 20.8 & 20.9 & 20.9 & 21.0 & 21.1 \\
\hline 68 & 20.0 & 20.1 & 20.1 & 20.2 & 20.2 & 20.3 & 20.3 & 20.4 & 20.4 & 20.5 \\
\hline 67 & 19.4 & 19.5 & 19.6 & 19.6 & 19.7 & 19.7 & 19.8 & 19.8 & 19.9 & 19.9 \\
\hline 66 & 18.9 & 18.9 & 19.0 & 19.1 & 19.1 & 19.2 & 19.2 & 19.3 & 19.3 & 19.4 \\
\hline 65 & 18.3 & 18.4 & 18.4 & 18.5 & 18.6 & 18.6 & 18.7 & 18.7 & 18.8 & 18.8 \\
\hline 64 & 17.8 & 17.8 & 17.9 & 17.9 & 18.0 & 18.1 & 18.1 & 18.2 & 18.2 & 18.3 \\
\hline 63 & 17.2 & 17.3 & 17.3 & 17.4 & 17.4 & 17.5 & 17.6 & 17.6 & 17.7 & 17.7 \\
\hline 62 & 16.7 & 16.7 & 16.8 & 16.8 & 16.9 & 16.9 & 17.0 & 17.1 & 17.1 & 17.2 \\
\hline 61 & 16.1 & 16.2 & 16.2 & 16.3 & 16.3 & 16.4 & 16.4 & 16.5 & 16.6 & 16.6 \\
\hline 60 & 15.6 & 15.6 & 15.7 & 15.7 & 15.8 & 15.8 & 15.9 & 15.9 & 16.0 & 16.1 \\
\hline 59 & 15.0 & $15 . I$ & 15.1 & 15.2 & 15.2 & 15.3 & $15 \cdot 3$ & 15.4 & 15.4 & 15.5 \\
\hline 58 & 14.4 & 14.5 & 14.6 & 14.6 & 14.7 & 14.7 & 14.8 & 14.8 & 14.9 & 14.9 \\
\hline 57 & 13.9 & 13.9 & 14.0 & 14.1 & 14.1 & 14.2 & 14.2 & 14.3 & 14.3 & 14.4 \\
\hline 56 & 13.3 & 13.4 & 13.4 & 13.5 & 13.6 & 13.6 & 13.7 & 13.7 & 13.8 & 13.8 \\
\hline 55 & 12.8 & 12.8 & 12.9 & 12.9 & 13.0 & 13.1 & 13.1 & 13.2 & 13.2 & 13.3 \\
\hline 54 & 12.2 & $12 \cdot 3$ & $12 \cdot 3$ & 12.4 & 12.4 & 12.5 & 12.6 & 12.6 & 12.7 & 12.7 \\
\hline 53 & 11.7 & 11.7 & 11.8 & 11.8 & 11.9 & 11.9 & 12.0 & 12.1 & 12.1 & 12.2 \\
\hline 52 & 11.1 & 11.2 & 11.2 & 11.3 & 11.3 & 11.4 & 11.4 & 11.5 & 11.6 & 11.6 \\
\hline 51 & 10.6 & 10.6 & 10.7 & 10.7 & 10.8 & 10.8 & 10.9 & 10.9 & 11.0 & 11.1 \\
\hline 50 & 10.0 & 10.1 & 10.1 & 10.2 & 10.2 & 10.3 & 10.3 & 10.4 & 10.4 & 10.5 \\
\hline
\end{tabular}




\section{TABIE 21 (Cont'd)}

\section{Temperature}

Conversion from Fahrenheit to Centigrade

\begin{tabular}{|c|c|c|c|c|c|c|c|c|c|c|}
\hline${ }^{\circ} F$ & 0.0 & 0.1 & 0.2 & 0.3 & 0.4 & 0.5 & 0.6 & 0.7 & 0.8 & 0.9 \\
\hline 49 & 09.4 & 09.5 & 09.6 & 09.6 & 09.7 & 09.7 & 09.8 & 09.8 & 09.9 & 09.9 \\
\hline 48 & 08.9 & 08.9 & 09.0 & 09.1 & 09.1 & 09.2 & 09.2 & 09.3 & 09.3 & 09.4 \\
\hline 47 & 08.3 & 08.4 & 08.4 & 08.5 & 08.6 & 08.6 & 08.7 & 08.7 & 08.8 & 08.8 \\
\hline 46 & 07.8 & 07.8 & 07.9 & 07.9 & 08.0 & 08.1 & 08.1 & 08.2 & 08.2 & 08.3 \\
\hline 45 & 07.2 & 07.3 & 07.3 & 07.4 & 07.4 & 07.5 & 07.6 & 07.6 & 07.7 & 07.7 \\
\hline 44 & 06.7 & 06.7 & 06.8 & 06.8 & 06.9 & 06.9 & 07.0 & 07.1 & 07.1 & 07.2 \\
\hline 43 & 06.1 & 06.2 & 06.2 & 06.3 & 06.3 & 06.4 & 06.4 & 06.5 & 06.6 & 06.6 \\
\hline 42 & 05.6 & 05.6 & 05.7 & 05.7 & 05.8 & 05.8 & 05.9 & 05.9 & 06.0 & 06.1 \\
\hline 41 & 05.0 & 05.1 & 05.1 & 05.2 & 05.2 & 05.3 & 05.3 & 05.4 & 05.4 & 05.5 \\
\hline 40 & 04.4 & 04.5 & 04.6 & 04.6 & 04.7 & 04.7 & 04.8 & 04.8 & 04.9 & 04.9 \\
\hline 39 & 03.9 & 03.9 & 04.0 & 04.1 & 04.1 & 04.2 & 04.2 & 04.3 & 04.3 & 04.4 \\
\hline 38 & 03.3 & 03.4 & 03.4 & 03.5 & 03.6 & 03.6 & 03.7 & 03.7 & 03.8 & 03.8 \\
\hline 37 & 02.8 & 02.8 & 02.9 & 02.9 & 03.0 & 03.1 & 03.1 & 03.2 & 03.2 & 03.3 \\
\hline 36 & 02.2 & 02.3 & 02.3 & 02.4 & 02.4 & 02.5 & 02.6 & 02.6 & 02.7 & 02.7 \\
\hline 35 & 01.7 & 01.7 & 01.8 & 01.8 & 01.9 & 01.9 & 02.0 & 02.1 & 02.1 & 02.2 \\
\hline 34 & 01.1 & 01.2 & 01.2 & 01.3 & 01.3 & 01.4 & 01.4 & 01.5 & 01.6 & 01.6 \\
\hline 33 & 00.6 & 00.6 & 00.7 & 00.7 & 00.8 & 00.8 & 00.9 & 00.9 & 01.0 & 01.1 \\
\hline 32 & 00.0 & 00.1 & 00.1 & 00.2 & 00.2 & 00.3 & 00.3 & 00.4 & 00.4 & 00.5 \\
\hline 31 & -00.6 & -00.5 & -00.4 & -00.4 & -00.3 & -00.3 & -00.2 & -00.2 & -00.1 & -00.1 \\
\hline 30 & -01.1 & -01.1 & -01.0 & -00.9 & -00.9 & -00.8 & -00.8 & -00.7 & -00.7 & -00.6 \\
\hline 29 & -01.7 & -01.6 & -01.6 & -01.5 & -01.4 & -01.4 & -01.3 & -01.3 & -01.2 & -01.2 \\
\hline 28 & -02.2 & -02.2 & -02.1 & -02.1 & -02.0 & -01.9 & -01.9 & -01.8 & -01.8 & -01.7 \\
\hline 27 & -02.8 & -02.7 & -02.7 & -02.6 & -02.6 & -02.5 & -02.4 & -02.4 & -02.3 & -02.3 \\
\hline 26 & -03.3 & -03.3 & -03.2 & -03.2 & -03.1 & -03.1 & -03.0 & -02.9 & -02.9 & -02.8 \\
\hline 25 & -03.9 & -03.8 & -03.8 & -03.7 & -03.7 & -03.6 & -03.6 & -03.5 & -03.4 & -03.4 \\
\hline 24 & -04.4 & -04.4 & -04.3 & -04.3 & -04.2 & -04.2 & -04.1 & -04.1 & -04.0 & -03.9 \\
\hline 23 & -05.0 & -04.9 & -04.9 & -04.8 & -04.8 & -04.7 & -04.7 & -04.6 & -04.6 & -04.5 \\
\hline
\end{tabular}


TABLE 21 (Cont'd)

Temperature

Conversion from Fahrenheit to Centigrade

\begin{tabular}{|c|c|c|c|c|c|c|c|c|c|c|}
\hline$\circ F$ & 0.0 & 0.1 & 0.2 & 0.3 & 0.4 & 0.5 & 0.6 & 0.7 & 0.8 & 0.9 \\
\hline 22 & -05.6 & -05.5 & -05.4 & -05.4 & -05.3 & -05.3 & -05.2 & -05.2 & -05.1 & -05.1 \\
\hline 21 & -06.1 & -06.1 & -06.0 & -05.9 & -05.9 & -05.9 & -05.8 & -05.7 & -05.7 & -05.6 \\
\hline 20 & -06.7 & -06.6 & -06.6 & -06.5 & -06.4 & -06.4 & -06.3 & -06.3 & -06.2 & -06.2 \\
\hline 19 & -07.2 & -08.2 & -07.1 & -07.1 & -07.0 & -06.9 & -06.9 & -06.8 & -06.8 & -06.7 \\
\hline 18 & -07.8 & -07.7 & -07.7 & -07.6 & -07.6 & -07.5 & -07.4 & -07.4 & -07.3 & -07.3 \\
\hline 17 & $-08 \cdot 3$ & $-08 \cdot 3$ & -08.2 & -08.2 & -08.1 & -08.1 & -08.0 & -07.9 & -07.9 & -07.8 \\
\hline 16 & -08.9 & -08.8 & -08.8 & -08.7 & -08.7 & -08.6 & -08.6 & -08.5 & -08.4 & -08.4 \\
\hline 15 & -09.4 & -09.4 & -09.3 & -09.3 & -09.2 & -09.2 & -09.1 & -09.1 & -09.0 & -08.9 \\
\hline 14 & -10.0 & -09.9 & -09.9 & -09.8 & -09.8 & -09.7 & -09.7 & -09.6 & -09.6 & -09.5 \\
\hline 13 & -10.6 & -10.5 & -10.4 & -10.4 & $-10 \cdot 3$ & $-10 \cdot 3$ & -10.2 & -10.2 & -10.1 & -10.1 \\
\hline 12 & -11.1 & -11.1 & -11.0 & -10.9 & -10.9 & -10.8 & -10.8 & -10.7 & -10.7 & -10.6 \\
\hline 11 & -11.7 & -11.6 & -11.6 & -11.5 & -11.4 & -11.4 & $-11 \cdot 3$ & -11.3 & -11.2 & -11.2 \\
\hline 10 & $-12 \cdot 2$ & -12.2 & -12.1 & -12.1 & -12.0 & -11.9 & -11.9 & -11.8 & -11.8 & -11.7 \\
\hline 9 & -12.8 & -12.7 & -12.7 & -12.6 & -12.6 & $-12 \cdot 5$ & -12.4 & $-12 \cdot 4$ & -12.3 & $-12 \cdot 3$ \\
\hline 8 & $-13 \cdot 3$ & $-13 \cdot 3$ & -13.2 & $-13 \cdot 2$ & -13.1 & -13.1 & -13.0 & $-12 \cdot 9$ & -12.9 & -12.8 \\
\hline 7 & $-13 \cdot 9$ & -13.8 & $-13 \cdot 8$ & -13.7 & -13.7 & -13.6 & -13.6 & -13.5 & -13.4 & -13.4 \\
\hline 6 & -14.4 & -14.4 & $-14 \cdot 3$ & $-14 \cdot 3$ & $-14 \cdot 2$ & -14.2 & -14.1 & -14.1 & -14.0 & -13.9 \\
\hline 5 & -15.0 & -14.9 & -14.9 & -14.8 & -14.8 & -14.7 & $-14 \cdot 7$ & -14.6 & -14.6 & $-14 \cdot 5$ \\
\hline 4 & -15.6 & $-15 \cdot 5$ & -15.4 & -15.4 & $-15 \cdot 3$ & $-15 \cdot 3$ & $-15 \cdot 2$ & -15.2 & -15.1 & -15.1 \\
\hline 3 & -16.1 & -16.1 & -16.0 & $-15 \cdot 9$ & -15.9 & $-15 \cdot 8$ & -15.8 & $-15 \cdot 7$ & -15.7 & -15.6 \\
\hline 2 & -16.7 & -16.6 & -16.6 & -16.5 & -16.4 & -16.4 & $-16 \cdot 3$ & $-16 \cdot 3$ & -16.2 & -16.2 \\
\hline 1 & $-17 \cdot 2$ & $-17 \cdot 2$ & -17.1 & $-17 \cdot 1$ & -17.0 & -16.9 & -16.9 & -16.8 & -16.8 & -16.7 \\
\hline 0 & $-17 \cdot 8$ & -17.7 & -17.7 & $-17 \cdot 6$ & -17.6 & $-17 \cdot 5$ & $-17 \cdot 4$ & $-17 \cdot 4$ & $-17 \cdot 3$ & $-17 \cdot 3$ \\
\hline-0 & -17.8 & -17.8 & $-17 \cdot 9$ & $-17 \cdot 9$ & -18.0 & -18.1 & -18.1 & $-18 \cdot 2$ & -18.2 & -18.3 \\
\hline-1 & $-18 \cdot 3$ & -18.4 & -18.4 & -18.5 & -18.6 & -18.6 & -18.7 & -18.7 & -18.8 & -18.8 \\
\hline-2 & -18.9 & $-18 \cdot 9$ & -19.0 & -19.1 & -19.1 & -19.2 & -19.2 & $-19 \cdot 3$ & -19.3 & -19.4 \\
\hline-3 & -19.4 & -19.5 & -19.6 & -19.6 & -19.7 & -19.7 & -19.8 & -19.8 & -19.9 & -19.9 \\
\hline
\end{tabular}




\section{TABLE 21 (Cont' $d$ )}

\section{Temperature}

Conversion from Fahrenheit to Centigrađe

\begin{tabular}{|c|c|c|c|c|c|c|c|c|c|c|}
\hline${ }^{\circ} \mathrm{F}$ & 0.0 & 0.1 & 0.2 & 0.3 & 0.4 & 0.5 & 0.6 & 0.7 & 0.8 & 0.9 \\
\hline-4 & -20.0 & -20.1 & -20.1 & -20.2 & -20.2 & $-20 \cdot 3$ & $-20 \cdot 3$ & -20.4 & -20.4 & $-20 \cdot 5$ \\
\hline-5 & -20.6 & -20.6 & -20.7 & -20.7 & -20.3 & -20.8 & -20.9 & -20.9 & $-21 \cdot 0$ & $-21 \cdot 1$ \\
\hline-6 & -21.1 & $-21 \cdot 2$ & $-21 \cdot 2$ & $-21 \cdot 3$ & $-21 \cdot 3$ & -21.4 & -21.4 & -21.5 & -21.6 & -21.6 \\
\hline-7 & $-21 \cdot 7$ & -21.7 & -21.8 & $-21 \cdot 8$ & $-21 \cdot 9$ & -21.9 & -22.0 & -22.1 & -22.1 & -22.2 \\
\hline-8 & $-22 \cdot 2$ & $-22 \cdot 3$ & $-22 \cdot 3$ & -22.4 & -22.4 & $-22 \cdot 5$ & $-22 \cdot 6$ & -22.6 & -22.7 & -22.7 \\
\hline-9 & $-22 \cdot 8$ & -22.8 & $-22 \cdot 9$ & $-22 \cdot 9$ & -23.0 & -23.1 & -23.1 & -23.2 & -23.2 & $-23 \cdot 3$ \\
\hline-10 & -23.3 & -23.4 & -23.4 & $-23 \cdot 5$ & -23.6 & -23.6 & $-23 \cdot 7$ & $-23 \cdot 7$ & -23.8 & -23.8 \\
\hline-11 & $-23 \cdot 9$ & -23.9 & -24.0 & -24.1 & -24.1 & $-24 \cdot 2$ & -24.2 & $-24 \cdot 3$ & $-24 \cdot 3$ & -24.4 \\
\hline-12 & -24.4 & $-24 \cdot 5$ & $-24 \cdot 6$ & -24.6 & -24.7 & -24.7 & -24.8 & -24.8 & $-24 \cdot 9$ & $-24 \cdot 9$ \\
\hline-13 & -25.0 & -25.1 & -25.1 & -25.2 & -25.2 & $-25 \cdot 3$ & $-25 \cdot 3$ & -25.4 & -25.4 & $-25 \cdot 5$ \\
\hline-14 & $-25 \cdot 6$ & -25.6 & $-25 \cdot 7$ & $-25 \cdot 7$ & -25.8 & -25.8 & -25.9 & -26.9 & -26.0 & -26.1 \\
\hline-15 & -26.1 & $-26 \cdot 2$ & -26.2 & $-26 \cdot 3$ & $-26 \cdot 3$ & -26.4 & -26.4 & $-26 \cdot 5$ & -26.6 & -26.6 \\
\hline-16 & -26.7 & -26.7 & -26.8 & -26.8 & $-26 \cdot 9$ & $-26 \cdot 9$ & & & & $-27 \cdot 2$ \\
\hline-17 & $-27 \cdot 2$ & $-27 \cdot 3$ & $-27 \cdot 3$ & $-27 \cdot 4$ & & $-27 \cdot 5$ & -27.6 & -27.6 & -27.7 & $-27 \cdot 7$ \\
\hline-18 & $-27 \cdot 8$ & -27.8 & $-27 \cdot 9$ & & -28.0 & -28.1 & -28.1 & & & -28.3 \\
\hline-19 & $-28 \cdot 3$ & -28.4 & -28.4 & $-28 \cdot 5$ & -28.6 & -28.6 & -28.7 & -28.7 & & -28.8 \\
\hline-20 & -28.9 & $-28 \cdot 9$ & $-29 \cdot 0$ & -29.1 & -29.1 & $-29 \cdot 2$ & $-29 \cdot 2$ & -29.3 & & $-29 \cdot 4$ \\
\hline-21 & $-29 \cdot 4$ & $-29 \cdot 5$ & -29.6 & $-29 \cdot 6$ & -29.7 & -29.7 & -29.8 & -29.8 & & $-29 \cdot 9$ \\
\hline-22 & -30.0 & -30.1 & -30.1 & -30.2 & -30.2 & -30.3 & -30.3 & -30.4 & -30.4 & -30.5 \\
\hline-23 & -30.6 & -30.6 & -30.7 & -30.7 & -30.8 & -30.8 & -30.9 & -30.9 & -31.0 & -31.1 \\
\hline-24 & -31.1 & -31.2 & -31.2 & -31.3 & $-31 \cdot 3$ & -31.4 & -31.4 & -31.6 & -31.6 & -31.6 \\
\hline-25 & -31.7 & -31.7 & -31.8 & -31.8 & $-31 \cdot 9$ & -31.9 & -32.0 & -32.1 & -32.1 & $-32 \cdot 2$ \\
\hline-26 & -32.2 & -32.3 & $-32 \cdot 3$ & -32.4 & -32.4 & -32.5 & -32.6 & -32.6 & -32.7 & -32.7 \\
\hline-27 & -32.8 & -32.8 & $-32 \cdot 9$ & -32.9 & -33.0 & -33.1 & -33.1 & -33.2 & -33.2 & $-33 \cdot 3$ \\
\hline-28 & $-33 \cdot 3$ & -33.4 & -33.4 & -33.5 & -33.6 & -33.6 & -33.7 & -33.7 & -33.8 & -33.8 \\
\hline-29 & -33.9 & -33.9 & -34.0 & -34.1 & -34.1 & -34.2 & -34.2 & -34.3 & -34.3 & -34.4 \\
\hline-30 & -34.4 & -34.5 & -34.6 & -34.6 & -34.7 & -34.7 & -34.8 & -34.8 & $-34 \cdot 9$ & -34.9 \\
\hline
\end{tabular}


TABIE 21 (Cont'd)

Temperature

Conversion from Fahrenheit to Centigrade

\begin{tabular}{|c|c|c|c|c|c|c|c|c|c|c|}
\hline${ }^{\circ} \mathrm{F}$ & 0.0 & 0.1 & 0.2 & 0.3 & 0.4 & 0.5 & 0.6 & 0.7 & 0.8 & 0.9 \\
\hline-31 & -35.0 & -35.1 & -35.1 & -35.2 & -35.2 & -35.3 & $-35 \cdot 3$ & -35.4 & -35.4 & $-35 \cdot 5$ \\
\hline-32 & -35.6 & -35.6 & -35.7 & -35.7 & -35.8 & -35.8 & -35.9 & -35.9 & -36.0 & -36.1 \\
\hline-33 & -36.1 & -36.2 & -36.2 & -36.3 & -36.3 & -36.4 & -36.4 & -36.5 & -36.6 & -36.6 \\
\hline-34 & -36.7 & -36.7 & -36.8 & -36.8 & -36.9 & -36.9 & -37.0 & -37.1 & -37.1 & $-37 \cdot 2$ \\
\hline-35 & $-37 \cdot 2$ & $-37 \cdot 3$ & $-37 \cdot 3$ & $-37 \cdot 4$ & -37.4 & $-37 \cdot 5$ & -37.6 & -37.6 & -37.7 & -37.7 \\
\hline-36 & -37.8 & -37.8 & -37.9 & -37.9 & -38.0 & -38.1 & -38.1 & -38.2 & -38.2 & $-38 \cdot 3$ \\
\hline-37 & $-38 \cdot 3$ & -38.4 & -38.4 & -38.4 & -38.5 & -38.6 & -38.6 & -38.7 & -38.7 & -38.8 \\
\hline-38 & -38.9 & -38.9 & -39.0 & -39.1 & -39.1 & -39.2 & -39.2 & $-39 \cdot 3$ & -39.3 & -39.4 \\
\hline-39 & -39.4 & $-39 \cdot 5$ & -39.6 & -39.6 & -39.7 & -39.7 & -39.8 & -39.8 & -39.9 & -39.9 \\
\hline-40 & -40.0 & -40.1 & -40.1 & -40.2 & -40.2 & -40.3 & -40.3 & -40.4 & -40.4 & -40.5 \\
\hline-41 & -40.6 & -40.6 & -40.7 & -40.7 & -40.8 & -40.8 & -40.9 & -40.9 & -41.0 & -41.1 \\
\hline-42 & -41.1 & $-41 \cdot 2$ & -41.2 & $-41 \cdot 3$ & $-41 \cdot 3$ & -41.4 & -41.4 & $-41 \cdot 5$ & -41.6 & $-41 \cdot 6$ \\
\hline-43 & -41.7 & -41.7 & -41.8 & -41.8 & $-41 \cdot 9$ & -41.9 & -42.0 & -42.1 & -42.1 & -42.2 \\
\hline-44 & -42.2 & $-42 \cdot 3$ & $-42 \cdot 3$ & -42.4 & -42.4 & -42.6 & -42.6 & -42.6 & -42.7 & -42.7 \\
\hline-45 & -42.8 & -42.8 & -42.9 & -42.9 & -43.0 & -43.1 & -43.1 & $-43 \cdot 2$ & $-43 \cdot 2$ & $-43 \cdot 3$ \\
\hline-46 & -43.3 & -43.4 & -43.4 & $-43 \cdot 5$ & -43.6 & -43.6 & -43.7 & -43.7 & -43.8 & -43.8 \\
\hline-47 & $-43 \cdot 9$ & -43.9 & -44.0 & -44.1 & -44.1 & -44.2 & -44.2 & -44.3 & $-44 \cdot 3$ & -44.4 \\
\hline-48 & -44.4 & $-44 \cdot 5$ & -44.6 & -44.6 & -44.7 & -44.7 & -44.8 & -44.8 & -44.9 & $-44 \cdot 9$ \\
\hline-49 & -45.0 & -45.1 & -45.1 & $-45 \cdot 2$ & $-45 \cdot 2$ & $-45 \cdot 3$ & $-45 \cdot 3$ & -45.4 & -45.4 & $-45 \cdot 5$ \\
\hline-50 & -45.6 & $-45 \cdot 6$ & -45.7 & $-45 \cdot 7$ & -45.8 & -45.8 & $-45 \cdot 9$ & $-45 \cdot 9$ & -46.0 & -46.1 \\
\hline-51 & -46.1 & -46.2 & -46.2 & $-46 \cdot 3$ & $-46 \cdot 3$ & -46.4 & -46.4 & $-46 \cdot 5$ & $-46 \cdot 5$ & -46.6 \\
\hline-52 & -46.7 & -46.7 & -46.8 & -46.8 & -46.9 & -46.9 & -47.0 & -47.1 & -47.1 & $-47 \cdot 2$ \\
\hline-55 & $-47 \cdot 2$ & $-47 \cdot 3$ & $-47 \cdot 3$ & $-47 \cdot 4$ & $-47 \cdot 4$ & $-47 \cdot 5$ & -47.6 & $-47 \cdot 6$ & $-47 \cdot 7$ & $-47 \cdot 7$ \\
\hline-54 & -47.8 & -47.8 & $-47 \cdot 9$ & $-47 \cdot 9$ & -48.0 & -48.1 & -48.1 & $-48 \cdot 2$ & $-48 \cdot 2$ & $-48 \cdot 3$ \\
\hline-55 & -48.3 & -48.4 & -48.4 & -48.5 & -48.6 & -48.6 & -48.7 & $-48 \cdot 7$ & -48.8 & -48.8 \\
\hline-56 & $-48 \cdot 9$ & $-48 \cdot 9$ & $-49 \cdot 0$ & -49.1 & -49.1 & -49.2 & $-49 \cdot 2$ & -49.3 & -49.3 & -49.4 \\
\hline-57 & -49.4 & $-49 \cdot 5$ & -49.6 & -49.6 & -49.7 & -49.7 & -49.8 & -49.8 & -49.9 & -49.9 \\
\hline
\end{tabular}


TABLE 2I (Cont'd)

Temperature

Conversion from Fahrenheit to Centigrade

\begin{tabular}{|c|c|c|c|c|c|c|c|c|c|c|}
\hline${ }^{\circ} \mathrm{F}$ & 0.0 & .1 & 0.2 & 0.3 & 0.4 & 0.5 & 0.6 & 0.7 & 0.8 & 0.9 \\
\hline-58 & -50.0 & -50.1 & -50.1 & -50.2 & -50.2 & -50.3 & -50.3 & -50.4 & -50.4 & -50.5 \\
\hline-59 & -50.6 & -50.6 & -50.7 & -50.7 & -50.8 & -50.8 & -50.9 & -50.9 & -51.0 & -51.1 \\
\hline-60 & -51.1 & -51.2 & -51.2 & -51.3 & -51.3 & -51.4 & -51.4 & -51.5 & -51.6 & -51.6 \\
\hline-61 & -51.7 & -51.7 & 1.8 & -51.8 & - & -51.9 & -52.0 & -52.1 & -52.1 & 52.2 \\
\hline-62 & -52.2 & $-52 \cdot 3$ & $-52 \cdot 3$ & -52.4 & -52.4 & -52.5 & & -52.6 & & -52.7 \\
\hline-63 & -52.8 & -52.8 & & & & & & & & -53.3 \\
\hline-64 & -53.3 & -53.4 & -53.4 & -53.5 & -53.6 & -53.6 & & -53.7 & & -53.8 \\
\hline-65 & -53.9 & -53 & -54.0 & -54.1 & -54 & -54 & & -5 & & $-54 \cdot 4$ \\
\hline-66 & -54.4 & -54.6 & -54.6 & -54.6 & -52 & $-54 \cdot 7$ & -54.8 & -54.8 & -54.9 & -54.9 \\
\hline-67 & -55.0 & -55.1 & -55.1 & -55.2 & -55 & -5 & -5 & -5 & -55.4 & -55.5 \\
\hline-68 & $-5 b \cdot 6$ & -55.6 & $-55 \cdot 7$ & -55.7 & -55.8 & -55.8 & & -55.9 & -56.0 & -56.1 \\
\hline-69 & -56.1 & -56.2 & -56.2 & -56.3 & -56.3 & -56.4 & & -56.5 & -56.6 & -56.6 \\
\hline-70 & -56.7 & -56.7 & -56.8 & -56.8 & -56.9 & -56.9 & -57.0 & -57.1 & -57.1 & -57.2 \\
\hline
\end{tabular}


TABLE 22

Present Weather

WMO Code 4501 for recording present weather

Code

figure

0 Clear (no cloud at any level)

1 Partly cloudy (scattered or broken)

2 Continuous layer(s) of cloud(s)

3 Sandstorm, duststorm, or blowing snow

4 Fog, thick dust or haze

5 Drizzle

6 Rain

7 Snow, or rain and snow mixed

8 Shower(s)

9 Thunderstorm(s) 
Present Weather

Conversion from Beaufort weather notation to WMO Code 4501

Abbreviation

b.

$\mathrm{bc}$.

c.

a.

e.

f.

fe.

g.

h.

$\mathrm{kq}$.

1 .

m.

o.

p.

q.

$r$.

rs.

s.

t.

tl.

u.

$\mathrm{v}$.

z.

\section{Description}

Code

Blue sky whether with clear or hazy atmosphere, or sky not more than one-quarter clouded.

Sky between one-quarter and three-quarters clouded.

Mainly cloudy (not less than three-quarters covered.)

Drizzle or fine rain.

Wet air without rain falling.

Fog.

Wet fog.

Gloomy

Hail.

Line squall.

Lightning

Mist.

Overcast sky (i.e., the whole sky covered

with unbroken cloud).

Passing showers.

Squalls.

Rain.

sleet (i.e., rain and snow together).

Snow.

Thunder.

Thunderstorm.

Ugly, threatening sky.

Unusual visibility.

Dust haze; the turbid atmosphere of dry weather. 
Present Weather

Conversion from 1936 International Meteorological Organization Code to the WMO Code 4501

Code Underlined

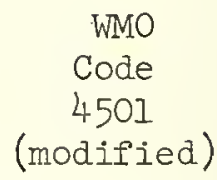

oo Cloudless

01 Partly cloudy.

O2 Cloudy.

03 Overcast.

04 Low fog, on ground or over sea.

05 Haze (but visibility greater than 2,000 m., 2,200 yds).

06 Dust devils seen.

07 Distant lightning. 1,100 and 2,200 yds).

09 Fog at a distance, but not at the ship.

10 Precipitation within sight.

11 Thunder, without precipitation at the ship.

12 Dust storm within sight, but not at the ship.

13 Ugly, threatening sky.

14 Squally weather.

$\begin{array}{l}15 \\ 16\end{array}$ Waterspouts seen. $\}$ in last 3 hours

08 Iight fog or mist (visibility between 1,000 and 2,000 m.,

PRECIPITATION IN LAST HOUR BUT NOT AT TIME OF OBSERVATION 
TABLE 24 (Cont'd)

Conversion from 1936 International Meteorological Organization Code to the WMO Code 4501

\section{DUST STORMS AND STORMS OF DRIFTING SNOW \\ (Visibility less than I,000 m., 1,100 yards)}

WMO
Code
4501
(modified)

30 Dust or sand storm.

31 Dust or sand storm, has decreased.

32 Dust or sand storm, no appreciable change.

33 Dust or sand storm, has increased.

34 Line of dust storms.

35 Storm of drifting snow.

\section{(Visibility less than $1,000 \mathrm{~m} ., 1,100$ yards)}

40 Fog.

41 Moderate fog in last hour
42 Thick fog in last hour but not at time of observation.

43 Fog, sky discernible has become thinner during last hour. 4

44 Fog, sky not discernible

$\begin{array}{l}45 \text { Fog, sky discernible } \\ 46\end{array}$ Fog, sky not discernible $\}$ no appreciable change during Iast hour

47 Fog, sky discernible has begun or become thicker during 4

48 Fog, sky not discernible last hour 4

49 Fog in patches.

\section{DRIZZIE}

(Precipitation consisting of numerous minute drops)

50 Drizzle

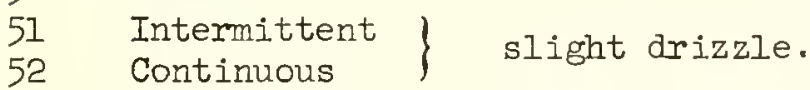

$\left.\begin{array}{ll}53 & \text { Intermittent } \\ 54 & \text { Continuous }\end{array}\right\}$ moderate drizzle.

$\left.\begin{array}{ll}55 & \text { Intermittent } \\ 56 & \text { Continuous }\end{array}\right\}$ thick drizzle.

57 Drizzle and fog.

$\left.\begin{array}{l}58 \text { Slight or moderate } \\ 59 \text { Thick }\end{array}\right\}$ drizzle and rain. 
TABLE 24 (Cont'd)

Conversion from 1936 International Meteorological Organization Code to the WMO Code 4501

\section{RAIN}

60 Rain.

$\left.\begin{array}{ll}61 & \begin{array}{l}\text { Intermittent } \\ 62\end{array}\end{array}\right\}$ slight rain.

$\left.\begin{array}{l}\text { Intermittent } \\ \text { Continuous }\end{array}\right\}$ moderate rain.

64

65 Intermittent $\}$ heavy rain.

67 Rain and fog.

$\left.\begin{array}{l}68 \text { Slight or moderate } \\ 69 \text { Heavy }\end{array}\right\}$ rain and snow, mixed.

SNOW

70 Snow or sleet

$\left.\begin{array}{ll}71 & \text { Intermittent } \\ 72 & \text { Continuous }\end{array}\right\}$ slight snow in flakes

$\left.\begin{array}{ll}73 & \text { Intermittent } \\ 74 & \text { Continuous }\end{array}\right\}$ moderate snow in flakes

$\left.\begin{array}{ll}75 & \text { Intermittent } \\ 76 & \text { Continuous }\end{array}\right\}$ heary snow in flakes

77 Snow and $\mathrm{eog}$.

78 Granular snow (frozen drizzle).

79 Ice crystals;

\section{SHOWERS ( $\mathrm{S}$ )}

80 Shower (s)

$\left.\begin{array}{ll}81 & \text { Shower (s) of slight or moderate } \\ 82 & \text { Shower (s) of heavy }\end{array}\right\}$ rain

(s) of slight or moderate

snow

85 Shower (s) of slight or moderate

86 Shower (s) of heavy

rain and snow.

87 Shower (s) of granular snow

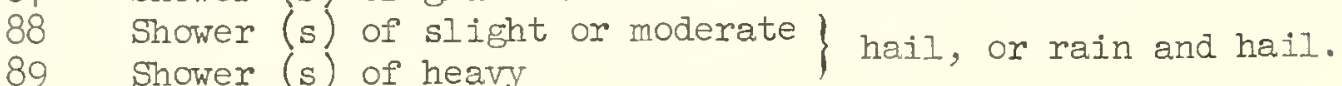


TABLE 24 (Cont'd)

Conversion from 1936 International Meteorological Organization Code to the WMO Code 4501

THUIDERSTORM

90 Thunderstorm

91 Rain at time Snow, or sleet

thunderstorm during last hour, but not at time of observation.

93 Thunderstorm, slight without hail or soft hail, but with rain (or snow)

94 Thunderstorm slight with soft hail

95 Thunderstorm moderate without hail, but with rain (or snow)

96 Thunderstorm moderate with soft hail

97 Thunderstorm heavy without hail, but with rain (or snow)

98 Thunderstorm combined with dust storm

99 Thunderstorm heavy with hail

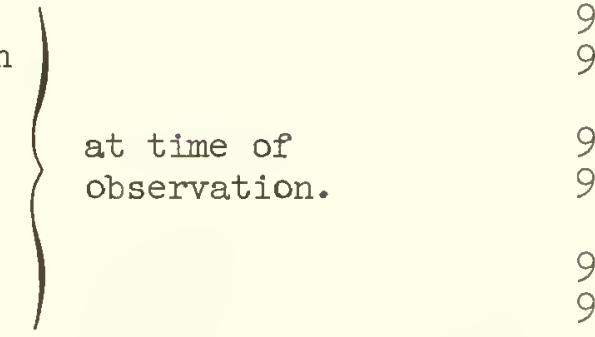




\section{Present Weather}

\section{WMO Code 4677 for recording present weather}

\section{Code figure}

WW

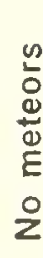

00 Cloud development not observed or not observable

01 Clouds generally dissolving or becoming less developed

02 State of sky on the whole unchanged

03 Clouds generally forming or developing

characteristic change of the state of sky during the past hour

04 Visibility reduced by smoke, e. g. veldt or forest fires, industrial smoke or volcanic ashes

05 Haze

06 Widespread dust in suspension in the air, not raised by wind at or near the station at the time of observation

07 Dust or sand raised by wind at or near the station at the time of observation, but no well developed dust whirl(s) or sand whirl(s), and no duststorm or sandstorm seen

08 Well developed dust whirl(s) or sand whirl(s) seen at or near the station during the preceding hour or at the time of observation, but no duststorm or sandstorm

09 Duststorm or sandstorm within sight at the time of observation, or at the station during the preceding hour

10 Mist

11 Patches of | shallow fog or ice fog at the station, whether on land or 12 More or less sea, not deeper than about 2 metres on land or 10 metres continuous at sea

13 Lightning visible, no thunder heard

14 Precipitation within sight, not reaching the ground or the surface of the sea

15 Precipitation within sight, reaching the ground or the surface of the sea, but distant (i.e. estimated to be more than $5 \mathrm{~km}$ ) from the station

16 Precipitation within sight, reaching the ground or the surface of the sea, near to, but not at the station

17 Thunderstorm, but no precipitation at the time of observation

18 Squalls | at or within sight of the station during the preceding

19 Funnel cloud(s)** $\}$ hour or at the time of observation

* The expression "at the station" refers to a land station or a ship.

* Tornado cloud or waterspout. 
$w w=20-29$ Precipitation, fog, ice fog or thunderstorm at the station during the preceding hour but not at the time of observation

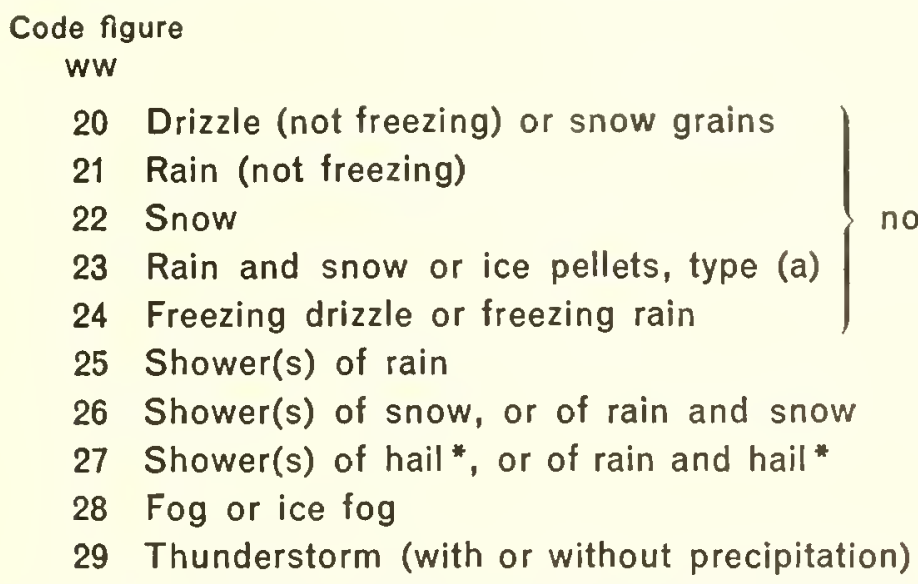

20 Drizzle (not freezing) or snow grains

21 Rain (not freezing)

22 Snow

23 Rain and snow or ice pellets, type (a)

24 Freezing drizzle or freezing rain

25 Shower(s) of rain

26 Shower(s) of snow, or of rain and snow

27 Shower(s) of hail * or of rain and hail *

28 Fog or ice fog

29 Thunderstorm (with or without precipitation)

$w w=30-39$ Duststorm, sandstorm, drifting or blowing snow

\begin{tabular}{|c|c|c|}
\hline & & \\
\hline 30 & & $\begin{array}{l}\text { - has decreased during the preceding } \\
\text { hour }\end{array}$ \\
\hline 31 & $\begin{array}{l}\text { Slight or moderate dust- } \\
\text { storm or sandstorm }\end{array}$ & $\begin{array}{l}\text { - no appreciable change during the } \\
\text { preceding hour }\end{array}$ \\
\hline 32 & & $\begin{array}{l}\text { - has begun or has increased during } \\
\text { the preceding hour }\end{array}$ \\
\hline 33 & & $\begin{array}{l}\text { - has decreased during the preceding } \\
\text { hour }\end{array}$ \\
\hline 34 & $\begin{array}{l}\text { Severe duststorm or } \\
\text { sandstorm }\end{array}$ & $\begin{array}{l}\text { - no appreciable change during the } \\
\text { preceding hour }\end{array}$ \\
\hline 35 & & $\begin{array}{l}\text { - has begun or has increased during } \\
\text { the preceding hour }\end{array}$ \\
\hline 36 & Slight or moderate blowing snow & generally low (helow eve level) \\
\hline 37 & Heavy drifting snow & generany low (Derow eye revei) \\
\hline 38 & Slight or moderate blowing snow & generally high (above eye level) \\
\hline 39 & Heavy blowing snow & \\
\hline
\end{tabular}

$w w=40-49$ Fog or ice fog at the time of observation

ww

40 Fog or ice fog at a distance at the time of observation, but not at the station during the preceding hour, the fog or ice fog extending to a level above that of the observer

41 Fog or ice fog in patches

42 Fog or ice fog, sky visible | has become thinner during the preceding

43 Fog or ice fog, sky invisible hour

* Hail, ice pellets, type (b), snow pellets. French: grêle, grésil ou neige roulée. 


\section{Code figure}

44 Fog or ice fog, sky visible | no appreciable change during the preced-

45 Fog or ice fog, sky invisible f ing hour

46 Fog or ice fog, sky visible | has begun or has become thicker during

47 Fog or ice fog, sky invisible $\}$ the preceding hour

48 Fog, depositing rime, sky visible

49 Fog, depositing rime, sky invisible

\section{$w w=50-99$ Precipitation at the station at the time of observation}

$w w=50-59$ Drizzle

WW

50 Drizzle, not freezing, intermittent

51 Drizzle, not freezing, continuous

slight at time of observation

52 Drizzle, not freezing, intermittent

53 Drizzle, not freezing, continuous

moderate at time of observation

54 Drizzle, not freezing, intermittent

55 Drizzle, not freezing, continuous f

heavy (dense) at time of observation

56 Drizzle, freezing, slight

57 Drizzle, freezing, moderate or heavy (dense)

58 Drizzle and rain, slight

59 Drizzle and rain, moderate or heavy

$w w=60-69$ Rain

WW

60 Rain, not freezing, intermittent

61 Rain, not freezing, continuous

62 Rain, not freezing, intermittent

63 Rain, not freezing, continuous

64 Rain, not freezing, intermittent

65 Rain, not freezing, continuous

66 Rain, freezing, slight

67 Rain, freezing, moderate or heavy

68 Rain or drizzle and snow, slight

69 Rain or drizzle and snow, moderate or heavy slight at time of observation

moderate at time of observation

heavy at time of observation

$w w=70-79$ Solid precipitation not in showers

WW

70 Intermittent fall of snow flakes

71 Continuous fall of snow flakes

72 Intermittent fall of snow flakes

73 Continuous fall of snow flakes slight at time of observation

moderate at time of observation 
Code figure

74 Intermittent fall of snow flakes I

75 Continuous fall of snow flakes f

heavy at time of observation

76 Ice prisms (with or without fog)

77 Snow grains (with or without fog)

78 Isolated starlike snow crystals (with or without fog)

79 Ice pellets, type (a)

$w w=80-99$ Showery precipitation, or precipitation with current or recent thunderstorm

WW

80 Rain shower(s), slight

81 Rain shower(s), moderate or heavy

82 Rain shower(s), violent

83 Shower(s) of rain and snow mixed, slight

84 Shower(s) of rain and snow mixed, moderate or heavy

85 Snow shower(s), slight

86 Snow shower(s), moderate or heavy

87 Shower(s) of snow pellets or ice

pellets, type (b), with or without

$88 \int$ rain or rain and snow mixed

- slight

89 Shower(s) of hail*, with or without rain or rain and snow

90 mixed, not associated with thunder

- moderate or heavy

- slight

- moderate or heavy

91 Slight rain at time of observation

92 Moderate or heavy rain at time of observation

93 Slight snow, or rain and snow mixed or hail** at time of observation

94 Moderate or heavy snow, or rain and snow mixed or hail** at time of observation

95 Thunderstorm, slight or moderate, without hail**, but with rain and/or snow at time of observation

96 Thunderstorm, slight or moderate, with hail** at time of observation

97 Thunderstorm, heavy, without hail"*, but with rain and/or snow at time of observation

thunderstorm during the preceding hour but not at time of observation

thunderstorm at time of observation

98 Thunderstorm combined with duststorm or sandstorm at time of observation

99 Thunderstorm, heavy, with hail** at time of observation

* French: grêle.

** Hail, ice pellets, type (b), snow pellets. French: grêle, grésil ou neige roulée. 


\section{TABLE 26}

Cloud Type (Genus)

WMO Cođe 0500 for recording cloud type (genus)

\section{Code}

o Cirrus......... $\mathrm{Ci}$

1 Cirrocumulus ...... Cc

2 Cirrostratus ....... Cs

3 Altocumulus ..... Ac

4 Altostratus ...... As

5 Nimbostratus ...... Ns

6 Stratocumulus...... Sc

7 Stratus ........ St

8 Cumulus........ $\mathrm{Cu}$

9 Cumulonimbus...... $\mathrm{Cb}$

$x$ Cloud not visible owing to darkness, fog, duststorm, sandstorm, or other analogous phenomena 


\section{TABLE 27}

Cloud Amount

WMO Code 2700 for recording cloud amount

\section{Code}

00

11 okta or less, but not zero

0

22 oktas

33 oktas

44 oktas

55 oktas

66 oktas

77 oktas or more, but not 8 oktas

88 oktas

$1 / 10$ or less, but not zero

$2 / 10-3 / 10$

$4 / 10$

$5 / 10$

$6 / 10$

$7 / 10-8 / 10$

$\% / 10$ or more, but not $10 / 10$

${ }^{10} / 10$

9 Sky obscured, or cloud amount cannot be estimated 


\section{TABLE 28}

\section{Visibility}

WMo code 4300 for recording visibility at surface

Code

0 Less than 50 metres (less than 55 yards)

$150-200$ metres (approx. 55-220 yards)

2 200-500 metres (approx. 220-550 yards)

3 500-1,000 metres (approx. 550 yards $-5 / 8$ n.m.)

4 1- $2 \mathrm{~km} \quad$ (approx. 5/8-1 n.m.)

5 2- $4 \mathrm{~km} \quad$ (approx. 1- 2 n.m.)

6 4-10 km (approx. 2- 6 n.m.)

7 10-20 km (approx. 6-12 n.m.)

8 20-50 km (approx. 12-30 n.m.)

$950 \mathrm{~km}$ or more (30 n.m. or more) 
TABIE 29

Precision of Measurement

This table is to be added later. 
TABIE 30

Salinity

Conversion from chlorinity to salinity $\%$

\begin{tabular}{|c|c|c|c|c|c|c|c|}
\hline $\mathrm{Cl}$ & $S$ & Cl & $\mathrm{S}$ & $\mathrm{Cl}$ & $S$ & $\mathrm{Cl}$ & $\mathrm{S}$ \\
\hline $\begin{array}{r}0.01 \\
.02 \\
.03 \\
.04 \\
.05 \\
.06 \\
.07 \\
.08 \\
.09 \\
0.10 \\
.11 \\
.12 \\
.13 \\
.14 \\
.15 \\
.16 \\
.17 \\
.18 \\
.19 \\
0.20 \\
.21 \\
.22 \\
.23 \\
.24 \\
.25 \\
.26 \\
.27 \\
.28 \\
.29 \\
0.30 \\
.31 \\
.32 \\
.33 \\
.34 \\
.35 \\
.36 \\
.37 \\
.38 \\
.39\end{array}$ & $\begin{array}{l}0.05 \\
.07 \\
.08 \\
.10 \\
.12 \\
.14 \\
.16 \\
.17 \\
.19 \\
0.21 \\
.23 \\
.25 \\
.26 \\
.28 \\
.30 \\
.32 \\
.34 \\
.35 \\
.37 \\
0.39 \\
.41 \\
.43 \\
.45 \\
.46 \\
.48 \\
.50 \\
.52 \\
.54 \\
.55 \\
0.57 \\
.59 \\
.61 \\
.63 \\
.64 \\
.66 \\
.68 \\
.70 \\
.72 \\
.73\end{array}$ & $\begin{array}{r}0.40 \\
.41 \\
.42 \\
.43 \\
.44 \\
.45 \\
.46 \\
.47 \\
.48 \\
.49 \\
0.50 \\
.51 \\
.52 \\
.53 \\
.54 \\
.55 \\
.56 \\
.57 \\
.58 \\
.59 \\
0.60 \\
.61 \\
.62 \\
.63 \\
.64 \\
.65 \\
.66 \\
.67 \\
.68 \\
.69 \\
0.70 \\
.71 \\
.72 \\
.73 \\
.74 \\
.75 \\
.76 \\
.78 \\
.79\end{array}$ & $\begin{array}{r}0.75 \\
.77 \\
.79 \\
.81 \\
.82 \\
.84 \\
.86 \\
.88 \\
.90 \\
.91 \\
0.93 \\
.95 \\
.97 \\
.99 \\
1.00 \\
1.02 \\
1.04 \\
1.06 \\
1.08 \\
1.09 \\
1.11 \\
1.13 \\
1.15 \\
1.17 \\
1.19 \\
1.20 \\
1.22 \\
1.24 \\
1.26 \\
1.28 \\
1.29 \\
1.31 \\
1.33 \\
1.35 \\
1.37 \\
1.38 \\
1.40 \\
1.42 \\
1.44 \\
1.46\end{array}$ & $\begin{array}{r}0.80 \\
.81 \\
.82 \\
.83 \\
.84 \\
.85 \\
.86 \\
.87 \\
.88 \\
.89 \\
0.90 \\
.91 \\
.92 \\
.93 \\
.94 \\
.95 \\
.96 \\
.97 \\
.98 \\
.99 \\
1.00 \\
.01 \\
.02 \\
.03 \\
.04 \\
.05 \\
.06 \\
.07 \\
.08 \\
.09 \\
1.10 \\
.11 \\
.12 \\
.13 \\
.14 \\
.15 \\
.16 \\
.17 \\
.19\end{array}$ & $\begin{array}{r}1.47 \\
.49 \\
.51 \\
.53 \\
.55 \\
.56 \\
.58 \\
.60 \\
.62 \\
.64 \\
1.65 \\
.67 \\
.69 \\
.71 \\
.73 \\
.74 \\
.76 \\
.78 \\
.80 \\
.82 \\
1.84 \\
.85 \\
.87 \\
.89 \\
.91 \\
.93 \\
.94 \\
.96 \\
.98 \\
2.00 \\
. .02 \\
.03 \\
.05 \\
.07 \\
.09 \\
.11 \\
.12 \\
.14 \\
.16 \\
.18\end{array}$ & $\begin{array}{r}1.20 \\
.21 \\
.22 \\
.23 \\
.24 \\
.25 \\
.26 \\
.27 \\
.28 \\
.29 \\
1.30 \\
.31 \\
.32 \\
.33 \\
.34 \\
.35 \\
.36 \\
.37 \\
.38 \\
.39\end{array}$ & $\begin{array}{r}2.20 \\
.21 \\
.23 \\
.25 \\
.27 \\
.29 \\
.30 \\
.32 \\
.34 \\
.36 \\
2.38 \\
.39 \\
.41 \\
.43 \\
.45 \\
.47 \\
.48 \\
.50 \\
.52 \\
.54\end{array}$ \\
\hline
\end{tabular}


TABLE 30 (Cont'd)

Salinfty

Conversion from chlorinity to salinity $(\%)$

\begin{tabular}{|c|c|c|c|c|c|c|c|}
\hline $\mathrm{Cl}$ & $S$ & $\mathrm{Cl}$ & S & $\mathrm{Cl}$ & S & $\mathrm{Cl}$ & S \\
\hline $\begin{array}{r}1.40 \\
.41 \\
.42 \\
.43 \\
.44 \\
.45 \\
.46 \\
.47 \\
.48 \\
.49\end{array}$ & $\begin{array}{r}2.56 \\
.58 \\
.59 \\
.61 \\
.63 \\
.65 \\
.67 \\
.68 \\
.70 \\
.72\end{array}$ & $\begin{array}{r}1.80 \\
.81 \\
.82 \\
.83 \\
.84 \\
.85 \\
.86 \\
.87 \\
.88 \\
.89\end{array}$ & $\begin{array}{l}3.28 \\
.30 \\
.32 \\
.33 \\
.35 \\
.37 \\
.39 \\
.41 \\
.42 \\
.44\end{array}$ & $\begin{array}{l}2.20 \\
.21 \\
.22 \\
.23 \\
.24 \\
.25 \\
.26 \\
.27 \\
.28 \\
.29\end{array}$ & $\begin{array}{l}4.00 \\
.02 \\
.04 \\
.06 \\
.07 \\
.09 \\
.11 \\
.13 \\
.15 \\
.16\end{array}$ & $\begin{array}{l}2.60 \\
.61 \\
.62 \\
.63 \\
.64 \\
.65 \\
.66 \\
.67 \\
.68 \\
.69\end{array}$ & $\begin{array}{r}4.72 \\
.74 \\
.76 \\
.78 \\
.80 \\
.81 \\
.83 \\
.85 \\
.87 \\
.89\end{array}$ \\
\hline $\begin{array}{l}1.50 \\
.51 \\
.52 \\
.53 \\
.54 \\
.55 \\
.56 \\
.57 \\
.58 \\
.59\end{array}$ & $\begin{array}{r}2.74 \\
.76 \\
.77 \\
.79 \\
.81 \\
.83 \\
.85 \\
.86 \\
.88 \\
.90\end{array}$ & $\begin{array}{r}1.90 \\
.91 \\
.92 \\
.93 \\
.94 \\
.95 \\
.96 \\
.97 \\
.98 \\
.99\end{array}$ & $\begin{array}{r}3.46 \\
.48 \\
.50 \\
.51 \\
.53 \\
.55 \\
.57 \\
.59 \\
.60 \\
.62\end{array}$ & $\begin{array}{r}2.30 \\
.31 \\
.32 \\
.33 \\
.34 \\
.35 \\
.36 \\
.37 \\
.38 \\
.39\end{array}$ & $\begin{array}{r}4.18 \\
.20 \\
.22 \\
.24 \\
.25 \\
.27 \\
.29 \\
.31 \\
.33 \\
.34\end{array}$ & $\begin{array}{r}2.70 \\
.71 \\
.72 \\
.73 \\
.74 \\
.75 \\
.76 \\
.77 \\
.78 \\
.79\end{array}$ & $\begin{array}{r}4.90 \\
.92 \\
.94 \\
.96 \\
.98 \\
.99 \\
5.01 \\
.03 \\
.05 \\
.07\end{array}$ \\
\hline $\begin{array}{r}1.60 \\
.61 \\
.62 \\
.63 \\
.64 \\
.65 \\
.66 \\
.67 \\
.68 \\
.69\end{array}$ & $\begin{array}{r}2.92 \\
.94 \\
.95 \\
.97 \\
.99 \\
3.01 \\
.03 \\
.04 \\
.06 \\
.08\end{array}$ & $\begin{array}{l}2.00 \\
.01 \\
.02 \\
.03 \\
.04 \\
.05 \\
.06 \\
.07 \\
.08 \\
.09\end{array}$ & $\begin{array}{l}3.64 \\
.66 \\
.68 \\
.69 \\
.71 \\
.73 \\
.75 \\
.77 \\
.78 \\
.80\end{array}$ & $\begin{array}{r}2.40 \\
.41 \\
.42 \\
.43 \\
.44 \\
.45 \\
.46 \\
.47 \\
.48 \\
.49\end{array}$ & $\begin{array}{r}4.36 \\
.38 \\
.40 \\
.42 \\
.43 \\
.45 \\
.47 \\
.49 \\
.51 \\
.52\end{array}$ & $\begin{array}{r}2.80 \\
.81 \\
.82 \\
.83 \\
.84 \\
.85 \\
.86 \\
.87 \\
.88 \\
.89\end{array}$ & $\begin{array}{r}5.08 \\
.10 \\
.12 \\
.14 \\
.16 \\
.17 \\
.19 \\
.21 \\
.23 \\
.25\end{array}$ \\
\hline $\begin{array}{l}1.70 \\
.71 \\
.72 \\
.73 \\
.74 \\
.75 \\
.76 \\
.77 \\
.78 \\
.79\end{array}$ & $\begin{array}{r}3.10 \\
.12 \\
.13 \\
.15 \\
.17 \\
.19 \\
.21 \\
.22 \\
.24 \\
.26\end{array}$ & $\begin{array}{l}2.10 \\
.11 \\
.12 \\
.13 \\
.14 \\
.15 \\
.16 \\
.17 \\
.18 \\
.19\end{array}$ & $\begin{array}{r}3.82 \\
.84 \\
.86 \\
.87 \\
.89 \\
.91 \\
.93 \\
.95 \\
.96 \\
.98\end{array}$ & $\begin{array}{r}2.50 \\
.51 \\
.52 \\
.53 \\
.54 \\
.55 \\
.56 \\
.57 \\
.58 \\
.59\end{array}$ & $\begin{array}{r}4.54 \\
.56 \\
.58 \\
.60 \\
.61 \\
.63 \\
.65 \\
.67 \\
.69 \\
.70\end{array}$ & $\begin{array}{r}2.90 \\
.91 \\
.92 \\
.93 \\
.94 \\
.95 \\
.96 \\
.97 \\
.98 \\
.99\end{array}$ & $\begin{array}{r}5.26 \\
.28 \\
.30 \\
.32 \\
.34 \\
.35 \\
.37 \\
.39 \\
.41 \\
.43\end{array}$ \\
\hline
\end{tabular}


TABLE 30 (Cont'd)

Salinfty

Conversion from chlorinity to salinity $(\% / 00)$

\begin{tabular}{|c|c|c|c|c|c|c|c|}
\hline $\mathrm{Cl}$ & S & $\mathrm{Cl}$ & S & $\mathrm{Cl}$ & S & $\mathrm{C} I$ & S \\
\hline $\begin{array}{l}3.00 \\
.01 \\
.02 \\
.03 \\
.04 \\
.05 \\
.06 \\
.07 \\
.08 \\
.09\end{array}$ & $\begin{array}{l}5.45 \\
.46 \\
.48 \\
.50 \\
.52 \\
.54 \\
.55 \\
.57 \\
.59 \\
.61\end{array}$ & $\begin{array}{r}3.40 \\
.41 \\
.42 \\
.43 \\
.44 \\
.45 \\
.46 \\
.47 \\
.48 \\
.49\end{array}$ & $\begin{array}{l}6.17 \\
.19 \\
.20 \\
.22 \\
.24 \\
.26 \\
.28 \\
.29 \\
.31 \\
.33\end{array}$ & $\begin{array}{r}3.80 \\
.81 \\
.82 \\
.83 \\
.84 \\
.85 \\
.86 \\
.87 \\
.88 \\
.89\end{array}$ & $\begin{array}{r}6.89 \\
.91 \\
.93 \\
.94 \\
.96 \\
.98 \\
7.00 \\
.02 \\
.03 \\
.05\end{array}$ & $\begin{array}{r}4.20 \\
.21 \\
.22 \\
.23 \\
.24 \\
.25 \\
.26 \\
.27 \\
.28 \\
.29\end{array}$ & $\begin{array}{l}7.61 \\
.63 \\
.65 \\
.67 \\
.68 \\
.70 \\
.72 \\
.74 \\
.76 \\
.77\end{array}$ \\
\hline $\begin{array}{l}3.10 \\
.11 \\
.12 \\
.13 \\
.14 \\
.15 \\
.16 \\
.17 \\
.18 \\
.19\end{array}$ & $\begin{array}{l}5.63 \\
.64 \\
.66 \\
.68 \\
.70 \\
.72 \\
.73 \\
.75 \\
.77 \\
.79\end{array}$ & $\begin{array}{l}3.50 \\
.51 \\
.52 \\
.53 \\
.54 \\
.55 \\
.56 \\
.57 \\
.58 \\
.59\end{array}$ & $\begin{array}{l}6.35 \\
.37 \\
.38 \\
.40 \\
.42 \\
.44 \\
.46 \\
.47 \\
.49 \\
.51\end{array}$ & $\begin{array}{r}3.90 \\
.91 \\
.92 \\
.93 \\
.94 \\
.95 \\
.96 \\
.97 \\
.98 \\
.99\end{array}$ & $\begin{array}{l}7.07 \\
.09 \\
.11 \\
.12 \\
.14 \\
.16 \\
.18 \\
.20 \\
.21 \\
.23\end{array}$ & $\begin{array}{r}4.30 \\
.31 \\
.32 \\
.33 \\
.34 \\
.35 \\
.36 \\
.37 \\
.38 \\
.39\end{array}$ & $\begin{array}{r}7.79 \\
.81 \\
.83 \\
.85 \\
.86 \\
.88 \\
.90 \\
.92 \\
.94 \\
.95\end{array}$ \\
\hline $\begin{array}{l}3.20 \\
.21 \\
.22 \\
.23 \\
.24 \\
.25 \\
.26 \\
.27 \\
.28 \\
.29\end{array}$ & $\begin{array}{l}5.81 \\
.82 \\
.84 \\
.86 \\
.88 \\
.90 \\
.91 \\
.93 \\
.95 \\
.97\end{array}$ & $\begin{array}{l}3.60 \\
.61 \\
.62 \\
.63 \\
.64 \\
.65 \\
.66 \\
.67 \\
.68 \\
.69\end{array}$ & $\begin{array}{l}6.53 \\
.55 \\
.56 \\
.58 \\
.60 \\
.62 \\
.64 \\
.65 \\
.67 \\
.69\end{array}$ & $\begin{array}{l}4.00 \\
.01 \\
.02 \\
.03 \\
.04 \\
.05 \\
.06 \\
.07 \\
.08 \\
.09\end{array}$ & $\begin{array}{l}7.25 \\
.27 \\
.29 \\
.30 \\
.32 \\
.34 \\
.36 \\
.38 \\
.39 \\
.41\end{array}$ & $\begin{array}{r}4.40 \\
.41 \\
.42 \\
.43 \\
.44 \\
.45 \\
.46 \\
.47 \\
.48 \\
.49\end{array}$ & $\begin{array}{r}7.97 \\
.99 \\
8.01 \\
.03 \\
.04 \\
.06 \\
.08 \\
.10 \\
.12 \\
.13\end{array}$ \\
\hline $\begin{array}{l}3.30 \\
.31 \\
.32 \\
.33 \\
.34 \\
.35 \\
.36 \\
.37 \\
.38 \\
.39\end{array}$ & $\begin{array}{l}5.99 \\
6.00 \\
.02 \\
.04 \\
.06 \\
.08 \\
.09 \\
.11 \\
.13 \\
.15\end{array}$ & $\begin{array}{l}3.70 \\
.71 \\
.72 \\
.73 \\
.74 \\
.75 \\
.76 \\
.77 \\
.78 \\
.79\end{array}$ & $\begin{array}{l}6.71 \\
.73 \\
.74 \\
.76 \\
.78 \\
.80 \\
.82 \\
.83 \\
.85 \\
.87\end{array}$ & $\begin{array}{r}4.10 \\
.11 \\
.12 \\
.13 \\
.14 \\
.15 \\
.16 \\
.17 \\
.18 \\
.19\end{array}$ & $\begin{array}{r}7.43 \\
.45 \\
.47 \\
.48 \\
.50 \\
.52 \\
.54 \\
.56 \\
.57 \\
.59\end{array}$ & $\begin{array}{r}4.50 \\
.51 \\
.52 \\
.53 \\
.54 \\
.55 \\
.56 \\
.57 \\
.58 \\
.59\end{array}$ & $\begin{array}{r}8.15 \\
.17 \\
.19 \\
.21 \\
.22 \\
.24 \\
.26 \\
.28 \\
.30 \\
.31\end{array}$ \\
\hline
\end{tabular}


TABLE 30 (Cont'd)

Salinity

Conversion from chlorinity to salinity $(\% / 00)$

\begin{tabular}{|c|c|c|c|c|c|c|c|}
\hline $\mathrm{Cl}$ & $\mathrm{S}$ & $\mathrm{Cl}$ & $\mathrm{S}$ & $\mathrm{Cl}$ & $S$ & $\mathrm{Cl}$ & $\mathrm{S}$ \\
\hline $\begin{array}{r}4.60 \\
.61 \\
.62 \\
.63 \\
.64 \\
.65 \\
.66 \\
.67 \\
.68 \\
.69\end{array}$ & $\begin{array}{r}8.33 \\
.35 \\
.37 \\
.39 \\
.41 \\
.42 \\
.44 \\
.46 \\
.48 \\
.50\end{array}$ & $\begin{array}{l}5.00 \\
.01 \\
.02 \\
.03 \\
.04 \\
.05 \\
.06 \\
.07 \\
.08 \\
.09\end{array}$ & $\begin{array}{r}9.06 \\
.07 \\
.09 \\
.11 \\
.13 \\
.15 \\
.16 \\
.18 \\
.20 \\
.22\end{array}$ & $\begin{array}{r}5.40 \\
.41 \\
.42 \\
.43 \\
.44 \\
.45 \\
.46 \\
.47 \\
.48 \\
.49\end{array}$ & $\begin{array}{r}9.78 \\
.80 \\
.81 \\
.83 \\
.85 \\
.87 \\
.89 \\
.90 \\
.92 \\
.94\end{array}$ & $\begin{array}{l}5.80 \\
.81 \\
.82 \\
.83 \\
.84 \\
.85 \\
.86 \\
.87 \\
.88 \\
.89\end{array}$ & $\begin{array}{r}10.50 \\
.52 \\
.54 \\
.55 \\
.57 \\
.59 \\
.61 \\
.63 \\
.64 \\
.66\end{array}$ \\
\hline $\begin{array}{r}4.70 \\
.71 \\
.72 \\
.73 \\
.74 \\
.75 \\
.76 \\
.77 \\
.78 \\
.79\end{array}$ & $\begin{array}{l}8.51 \\
.53 \\
.55 \\
.57 \\
.59 \\
.60 \\
.62 \\
.64 \\
.66 \\
.68\end{array}$ & $\begin{array}{l}5.10 \\
.11 \\
.12 \\
.13 \\
.14 \\
.15 \\
.16 \\
.17 \\
.18 \\
.19\end{array}$ & $\begin{array}{r}9.24 \\
.25 \\
.27 \\
.29 \\
.31 \\
.33 \\
.34 \\
.36 \\
.38 \\
.40\end{array}$ & $\begin{array}{r}5.50 \\
.51 \\
.52 \\
.53 \\
.54 \\
.55 \\
.56 \\
.57 \\
.58 \\
.59\end{array}$ & $\begin{array}{r}9.96 \\
.98 \\
.99 \\
10.01 \\
.03 \\
.05 \\
.07 \\
.08 \\
.10 \\
.12\end{array}$ & $\begin{array}{r}5.90 \\
.91 \\
.92 \\
.93 \\
.94 \\
.95 \\
.96 \\
.97 \\
.98 \\
.99\end{array}$ & $\begin{array}{r}10.68 \\
.70 \\
.72 \\
.73 \\
.75 \\
.77 \\
.79 \\
.81 \\
.82 \\
.84\end{array}$ \\
\hline $\begin{array}{r}4.80 \\
.81 \\
.82 \\
.83 \\
.84 \\
.85 \\
.86 \\
.87 \\
.88 \\
.89\end{array}$ & $\begin{array}{r}8.69 \\
.71 \\
.73 \\
.75 \\
.77 \\
.78 \\
.80 \\
.82 \\
.84 \\
.86\end{array}$ & $\begin{array}{r}5.20 \\
.21 \\
.22 \\
.23 \\
.24 \\
.25 \\
.26 \\
.27 \\
.28 \\
.29\end{array}$ & $\begin{array}{r}9.42 \\
.43 \\
.45 \\
.47 \\
.49 \\
.51 \\
.52 \\
.54 \\
.56 \\
.58\end{array}$ & $\begin{array}{l}5.60 \\
.61 \\
.62 \\
.63 \\
.64 \\
.65 \\
.66 \\
.67 \\
.68 \\
.69\end{array}$ & $\begin{array}{r}10.14 \\
.16 \\
.17 \\
.19 \\
.21 \\
.23 \\
.25 \\
.26 \\
.28 \\
.30\end{array}$ & $\begin{array}{l}6.00 \\
.01 \\
.02 \\
.03 \\
.04 \\
.05 \\
.06 \\
.07 \\
.08 \\
.09\end{array}$ & $\begin{array}{r}10.86 \\
.88 \\
.90 \\
.91 \\
.93 \\
.95 \\
.97 \\
.99 \\
11.00 \\
.02\end{array}$ \\
\hline $\begin{array}{r}4.90 \\
.91 \\
.92 \\
.93 \\
.94 \\
.95 \\
.96 \\
.97 \\
.98 \\
.99\end{array}$ & $\begin{array}{r}8.87 \\
.89 \\
.91 \\
.93 \\
.95 \\
.96 \\
.98 \\
9.00 \\
.02 \\
.04\end{array}$ & $\begin{array}{l}5.30 \\
.31 \\
.32 \\
.33 \\
.34 \\
.35 \\
.36 \\
.37 \\
.38 \\
.39\end{array}$ & $\begin{array}{l}9.60 \\
.61 \\
.63 \\
.65 \\
.67 \\
.69 \\
.70 \\
.72 \\
.74 \\
.76\end{array}$ & $\begin{array}{l}5.70 \\
.71 \\
.72 \\
.73 \\
.74 \\
.75 \\
.76 \\
.77 \\
.78 \\
.79\end{array}$ & $\begin{array}{r}10.32 \\
.34 \\
.35 \\
.37 \\
.39 \\
.41 \\
.43 \\
.44 \\
.46 \\
.48\end{array}$ & $\begin{array}{r}6.10 \\
.11 \\
.12 \\
.13 \\
.14 \\
.15 \\
.16 \\
.17 \\
.18 \\
.19\end{array}$ & $\begin{array}{r}11.04 \\
.06 \\
.08 \\
.09 \\
.11 \\
.13 \\
.15 \\
.17 \\
.18 \\
.20\end{array}$ \\
\hline
\end{tabular}


TABLE 30 (Cont'a)

Salinity

Conversion from chlorinity to salinity $(\%)$

\begin{tabular}{|c|c|c|c|c|c|c|c|}
\hline $\mathrm{Cl}$ & $S$ & $\mathrm{Cl}$ & S & $\mathrm{Cl}$ & S & $\mathrm{Cl}$ & S \\
\hline $\begin{array}{l}6.20 \\
.21 \\
.22 \\
.23 \\
.24 \\
.25 \\
.26 \\
.27 \\
.28 \\
.29\end{array}$ & $\begin{array}{r}11.22 \\
.24 \\
.26 \\
.28 \\
.29 \\
.31 \\
.33 \\
.35 \\
.37 \\
.38\end{array}$ & $\begin{array}{l}6.60 \\
.61 \\
.62 \\
.63 \\
.64 \\
.65 \\
.66 \\
.67 \\
.68 \\
.69\end{array}$ & $\begin{array}{r}11.94 \\
.96 \\
.98 \\
12.00 \\
.02 \\
.03 \\
.05 \\
.07 \\
.09 \\
.11\end{array}$ & $\begin{array}{l}7.00 \\
.01 \\
.02 \\
.03 \\
.04 \\
.05 \\
.06 \\
.07 \\
.08 \\
.09\end{array}$ & $\begin{array}{r}12.67 \\
.68 \\
.70 \\
.72 \\
.74 \\
.76 \\
.77 \\
.79 \\
.81 \\
.83\end{array}$ & $\begin{array}{l}7.40 \\
.41 \\
.42 \\
.43 \\
.44 \\
.45 \\
.46 \\
.47 \\
.48 \\
.49\end{array}$ & $\begin{array}{r}13.39 \\
.41 \\
.42 \\
.44 \\
.46 \\
.48 \\
.50 \\
.51 \\
.53 \\
.55\end{array}$ \\
\hline $\begin{array}{l}6.30 \\
.31 \\
.32 \\
.33 \\
.34 \\
.35 \\
.36 \\
.37 \\
.38 \\
.39\end{array}$ & $\begin{array}{r}11.40 \\
.42 \\
.44 \\
.46 \\
.47 \\
.49 \\
.51 \\
.53 \\
.55 \\
.56\end{array}$ & $\begin{array}{l}6.70 \\
.71 \\
.72 \\
.73 \\
.74 \\
.75 \\
.76 \\
.77 \\
.78 \\
.79\end{array}$ & $\begin{array}{r}12.12 \\
.14 \\
.16 \\
.18 \\
.20 \\
.21 \\
.23 \\
.25 \\
.27 \\
.29\end{array}$ & $\begin{array}{r}7.10 \\
.11 \\
.12 \\
.13 \\
.14 \\
.15 \\
.16 \\
.17 \\
.18 \\
.19\end{array}$ & $\begin{array}{r}12.85 \\
.86 \\
.88 \\
.90 \\
.92 \\
.94 \\
.95 \\
.97 \\
.99 \\
13.01\end{array}$ & $\begin{array}{r}7.50 \\
.51 \\
.52 \\
.53 \\
.54 \\
.55 \\
.56 \\
.57 \\
.58 \\
.59\end{array}$ & $\begin{array}{r}13.57 \\
.59 \\
.60 \\
.62 \\
.64 \\
.66 \\
.68 \\
.69 \\
.71 \\
.73\end{array}$ \\
\hline $\begin{array}{l}6.40 \\
.41 \\
.42 \\
.43 \\
.44 \\
.45 \\
.46 \\
.47 \\
.48 \\
.49\end{array}$ & $\begin{array}{r}11.58 \\
.60 \\
.62 \\
.64 \\
.65 \\
.67 \\
.69 \\
.71 \\
.73 \\
.74\end{array}$ & $\begin{array}{l}6.80 \\
.81 \\
.82 \\
.83 \\
.84 \\
.85 \\
.86 \\
.87 \\
.88 \\
.89\end{array}$ & $\begin{array}{r}12.30 \\
.32 \\
.34 \\
.36 \\
.38 \\
.39 \\
.41 \\
.43 \\
.45 \\
.47\end{array}$ & $\begin{array}{r}7.20 \\
.21 \\
.22 \\
.23 \\
.24 \\
.25 \\
.26 \\
.27 \\
.28 \\
.29\end{array}$ & $\begin{array}{r}13.03 \\
.04 \\
.06 \\
.08 \\
.10 \\
.12 \\
.13 \\
.15 \\
.17 \\
.19\end{array}$ & $\begin{array}{l}7.60 \\
.61 \\
.62 \\
.63 \\
.64 \\
.65 \\
.66 \\
.67 \\
.68 \\
.69\end{array}$ & $\begin{array}{r}13.75 \\
.77 \\
.78 \\
.80 \\
.82 \\
.84 \\
.86 \\
.87 \\
.89 \\
.91\end{array}$ \\
\hline $\begin{array}{l}6.50 \\
.51 \\
.52 \\
.53 \\
.54 \\
.55 \\
.56 \\
.57 \\
.58 \\
.59\end{array}$ & $\begin{array}{r}11.76 \\
.78 \\
.80 \\
.82 \\
.83 \\
.85 \\
.87 \\
.89 \\
.91 \\
.92\end{array}$ & $\begin{array}{l}6.90 \\
.91 \\
.92 \\
.93 \\
.94 \\
.95 \\
.96 \\
.97 \\
.98 \\
.99\end{array}$ & $\begin{array}{r}12.48 \\
.50 \\
.52 \\
.54 \\
.56 \\
.57 \\
.59 \\
.61 \\
.63 \\
.65\end{array}$ & $\begin{array}{r}7.30 \\
.31 \\
.32 \\
.33 \\
.34 \\
.35 \\
.36 \\
.37 \\
.38 \\
.39\end{array}$ & $\begin{array}{r}13.21 \\
.22 \\
.24 \\
.26 \\
.28 \\
.30 \\
.31 \\
.33 \\
.35 \\
.37\end{array}$ & $\begin{array}{l}7.70 \\
.71 \\
.72 \\
.73 \\
.74 \\
.75 \\
.76 \\
.77 \\
.78 \\
.79\end{array}$ & $\begin{array}{r}13.93 \\
.95 \\
.96 \\
.98 \\
14.00 \\
.02 \\
.04 \\
.05 \\
.07 \\
.09\end{array}$ \\
\hline
\end{tabular}


TABLE 30 (Cont' $d$ )

Salinity

Conversion from chlorinity to salinity $(\% / 00)$

\begin{tabular}{|c|c|c|c|c|c|c|c|}
\hline $\mathrm{Cl}$ & S & $\mathrm{Cl}$ & S & $\mathrm{Cl}$ & S & $\mathrm{Cl}$ & S \\
\hline $\begin{array}{r}7.80 \\
.81 \\
.82 \\
.83 \\
.84 \\
.85 \\
.86 \\
.87 \\
.88 \\
.89\end{array}$ & $\begin{array}{r}14.11 \\
.13 \\
.15 \\
.16 \\
.18 \\
.20 \\
.22 \\
.24 \\
.25 \\
.27\end{array}$ & $\begin{array}{r}8.20 \\
.21 \\
.22 \\
.23 \\
.24 \\
.25 \\
.26 \\
.27 \\
.28 \\
.29\end{array}$ & $\begin{array}{r}14.83 \\
.85 \\
.87 \\
.89 \\
.90 \\
.92 \\
.94 \\
.96 \\
.98 \\
.99\end{array}$ & $\begin{array}{l}8.60 \\
.61 \\
.62 \\
.63 \\
.64 \\
.65 \\
.66 \\
.67 \\
.68 \\
.69\end{array}$ & $\begin{array}{r}15.55 \\
.57 \\
.59 \\
.61 \\
.63 \\
.64 \\
.66 \\
.68 \\
.70 \\
.72\end{array}$ & $\begin{array}{l}9.00 \\
.01 \\
.02 \\
.03 \\
.04 \\
.05 \\
.06 \\
.07 \\
.08 \\
.09\end{array}$ & $\begin{array}{r}16.28 \\
.29 \\
.31 \\
.33 \\
.35 \\
.37 \\
.38 \\
.40 \\
.42 \\
.44\end{array}$ \\
\hline $\begin{array}{r}7.90 \\
.91 \\
.92 \\
.93 \\
.94 \\
.95 \\
.96 \\
.97 \\
.98 \\
.99\end{array}$ & $\begin{array}{r}14.29 \\
.31 \\
.33 \\
.34 \\
.36 \\
.38 \\
.40 \\
.42 \\
.43 \\
.45\end{array}$ & $\begin{array}{r}8.30 \\
.31 \\
.32 \\
.33 \\
.34 \\
.35 \\
.36 \\
.37 \\
.38 \\
.39\end{array}$ & $\begin{array}{r}15.01 \\
.03 \\
.05 \\
.07 \\
.08 \\
.10 \\
.12 \\
.14 \\
.16 \\
.17\end{array}$ & $\begin{array}{r}8.70 \\
.71 \\
.72 \\
.73 \\
.74 \\
.75 \\
.76 \\
.77 \\
.78 \\
.79\end{array}$ & $\begin{array}{r}15.73 \\
.75 \\
.77 \\
.79 \\
.81 \\
.82 \\
.84 \\
.86 \\
.88 \\
.90\end{array}$ & $\begin{array}{l}9.10 \\
.11 \\
.12 \\
.13 \\
.14 \\
.15 \\
.16 \\
.17 \\
.18 \\
.19\end{array}$ & $\begin{array}{r}16.46 \\
.47 \\
.49 \\
.51 \\
.53 \\
.55 \\
.56 \\
.58 \\
.60 \\
.62\end{array}$ \\
\hline $\begin{array}{l}8.00 \\
.01 \\
.02 \\
.03 \\
.04 \\
.05 \\
.06 \\
.07 \\
.08 \\
.09\end{array}$ & $\begin{array}{r}14.47 \\
.49 \\
.51 \\
.52 \\
.54 \\
.56 \\
.58 \\
.60 \\
.61 \\
.63\end{array}$ & $\begin{array}{r}8.40 \\
.41 \\
.42 \\
.43 \\
.44 \\
.45 \\
.46 \\
.47 \\
.48 \\
.49\end{array}$ & $\begin{array}{r}15.19 \\
.21 \\
.23 \\
.25 \\
.26 \\
.28 \\
.30 \\
.32 \\
.34 \\
.35\end{array}$ & $\begin{array}{r}8.80 \\
.81 \\
.82 \\
.83 \\
.84 \\
.85 \\
.86 \\
.87 \\
.88 \\
.89\end{array}$ & $\begin{array}{r}15.91 \\
.93 \\
.95 \\
.97 \\
.99 \\
16.00 \\
.02 \\
.04 \\
.06 \\
.08\end{array}$ & $\begin{array}{r}9.20 \\
.21 \\
.22 \\
.23 \\
.24 \\
.25 \\
.26 \\
.27 \\
.28 \\
.29\end{array}$ & $\begin{array}{r}16.64 \\
.65 \\
.67 \\
.69 \\
.71 \\
.73 \\
.74 \\
.76 \\
.78 \\
.80\end{array}$ \\
\hline $\begin{array}{r}0.10 \\
.11 \\
.12 \\
.13 \\
.14 \\
.15 \\
.16 \\
.17 \\
.18 \\
.19\end{array}$ & $\begin{array}{r}14.65 \\
.67 \\
.69 \\
.70 \\
.72 \\
.74 \\
.76 \\
.78 \\
.79 \\
.81\end{array}$ & $\begin{array}{r}8.50 \\
.51 \\
.52 \\
.53 \\
.54 \\
.55 \\
.56 \\
.57 \\
.58 \\
.59\end{array}$ & $\begin{array}{r}15.37 \\
.39 \\
.41 \\
.43 \\
.44 \\
.46 \\
.48 \\
.50 \\
.52 \\
.53\end{array}$ & $\begin{array}{r}8.90 \\
.91 \\
.92 \\
.93 \\
.94 \\
.95 \\
.96 \\
.97 \\
.98 \\
.99\end{array}$ & $\begin{array}{r}16.09 \\
.11 \\
.13 \\
.15 \\
.17 \\
.18 \\
.20 \\
.22 \\
.24 \\
.26\end{array}$ & $\begin{array}{r}9.30 \\
.31 \\
.32 \\
.33 \\
.34 \\
.35 \\
.36 \\
.37 \\
.38 \\
.39\end{array}$ & $\begin{array}{r}16.82 \\
.83 \\
.85 \\
.87 \\
.89 \\
.91 \\
.92 \\
.94 \\
.96 \\
.98\end{array}$ \\
\hline
\end{tabular}


TABIE 30 (Cont'd)

Salinity

Conversion from chlorinity to salinity $(\% / 00)$

\begin{tabular}{|c|c|c|c|c|c|c|c|}
\hline $\mathrm{CI}$ & $S$ & $\mathrm{Cl}$ & $S$ & $\mathrm{Cl}$ & S & $\mathrm{Cl}$ & $\mathrm{S}$ \\
\hline $\begin{array}{l}9.40 \\
.41 \\
.42 \\
.43 \\
.44 \\
.45 \\
.46 \\
.47 \\
.48 \\
.49\end{array}$ & $\begin{array}{r}17.00 \\
.02 \\
.03 \\
.05 \\
.07 \\
.09 \\
.11 \\
.12 \\
.14 \\
.16\end{array}$ & $\begin{array}{r}9.80 \\
.81 \\
.82 \\
.83 \\
.84 \\
.85 \\
.86 \\
.87 \\
.88 \\
.89\end{array}$ & $\begin{array}{r}17.72 \\
.74 \\
.76 \\
.77 \\
.79 \\
.81 \\
.83 \\
.85 \\
.86 \\
.88\end{array}$ & $\begin{array}{r}10.20 \\
.21 \\
.22 \\
.23 \\
.24 \\
.25 \\
.26 \\
.27 \\
.28 \\
.29\end{array}$ & $\begin{array}{r}18.44 \\
.46 \\
.48 \\
.50 \\
.51 \\
.53 \\
.55 \\
.57 \\
.59 \\
.60\end{array}$ & $\begin{array}{r}10.60 \\
.61 \\
.62 \\
.63 \\
.64 \\
.65 \\
.66 \\
.67 \\
.68 \\
.69\end{array}$ & $\begin{array}{r}19.16 \\
.18 \\
.20 \\
.22 \\
.24 \\
.25 \\
.27 \\
.29 \\
.31 \\
.33\end{array}$ \\
\hline $\begin{array}{l}9.50 \\
.51 \\
.52 \\
.53 \\
.54 \\
.55 \\
.56 \\
.57 \\
.58 \\
.59\end{array}$ & $\begin{array}{r}17.18 \\
.20 \\
.21 \\
.23 \\
.25 \\
.27 \\
.29 \\
.30 \\
.32 \\
.34\end{array}$ & $\begin{array}{r}9.90 \\
.91 \\
.92 \\
.93 \\
.94 \\
.95 \\
.96 \\
.97 \\
.98 \\
.99\end{array}$ & $\begin{array}{r}17.90 \\
.92 \\
.94 \\
.95 \\
.97 \\
.99 \\
18.01 \\
.03 \\
.04 \\
.06\end{array}$ & $\begin{array}{r}10.30 \\
.31 \\
.32 \\
.33 \\
.34 \\
.35 \\
.36 \\
.37 \\
.38 \\
.39\end{array}$ & $\begin{array}{r}18.62 \\
.64 \\
.66 \\
.68 \\
.69 \\
.71 \\
.73 \\
.75 \\
.77 \\
.78\end{array}$ & $\begin{array}{r}10.70 \\
.71 \\
.72 \\
.73 \\
.74 \\
.75 \\
.76 \\
.77 \\
.78 \\
.79\end{array}$ & $\begin{array}{r}19.34 \\
.36 \\
.38 \\
.40 \\
.42 \\
.43 \\
.45 \\
.47 \\
.49 \\
.51\end{array}$ \\
\hline $\begin{array}{r}9.60 \\
.61 \\
.62 \\
.63 \\
.64 \\
.65 \\
.66 \\
.67 \\
.68 \\
.69\end{array}$ & $\begin{array}{r}17.36 \\
.38 \\
.39 \\
.41 \\
.43 \\
.45 \\
.47 \\
.48 \\
.50 \\
.52\end{array}$ & $\begin{array}{r}10.00 \\
.01 \\
.02 \\
.03 \\
.04 \\
.05 \\
.06 \\
.07 \\
.08 \\
.09\end{array}$ & $\begin{array}{r}18.08 \\
.10 \\
.12 \\
.13 \\
.15 \\
.17 \\
.19 \\
.21 \\
.22 \\
.24\end{array}$ & $\begin{array}{r}10.40 \\
.41 \\
.42 \\
.43 \\
.44 \\
.45 \\
.46 \\
.47 \\
.48 \\
.49\end{array}$ & $\begin{array}{r}18.80 \\
.82 \\
.84 \\
.86 \\
.87 \\
.89 \\
.91 \\
.93 \\
.95 \\
.96\end{array}$ & $\begin{array}{r}10.80 \\
.81 \\
.82 \\
.83 \\
.84 \\
.85 \\
.86 \\
.87 \\
.88 \\
.89\end{array}$ & $\begin{array}{r}19.52 \\
.54 \\
.56 \\
.58 \\
.60 \\
.61 \\
.63 \\
.65 \\
.67 \\
.69\end{array}$ \\
\hline $\begin{array}{r}9.70 \\
.71 \\
.72 \\
.73 \\
.74 \\
.75 \\
.76 \\
.77 \\
.78 \\
.79\end{array}$ & $\begin{array}{r}17.54 \\
.56 \\
.57 \\
.59 \\
.61 \\
.63 \\
.65 \\
.66 \\
.68 \\
.70\end{array}$ & $\begin{array}{r}10.10 \\
.11 \\
.12 \\
.13 \\
.14 \\
.15 \\
.16 \\
.17 \\
.18 \\
.19\end{array}$ & $\begin{array}{r}18.26 \\
.28 \\
.30 \\
.31 \\
.33 \\
.35 \\
.37 \\
.39 \\
.40 \\
.42\end{array}$ & $\begin{array}{r}10.50 \\
.51 \\
.52 \\
.53 \\
.54 \\
.55 \\
.56 \\
.57 \\
.58 \\
.59\end{array}$ & $\begin{array}{r}18.98 \\
19.00 \\
.02 \\
.04 \\
.05 \\
.07 \\
.09 \\
.11 \\
.13 \\
.14\end{array}$ & $\begin{array}{r}10.90 \\
.91 \\
.92 \\
.93 \\
.94 \\
.95 \\
.96 \\
.97 \\
.98 \\
.99\end{array}$ & $\begin{array}{r}19.70 \\
.72 \\
.74 \\
.76 \\
.78 \\
.79 \\
.81 \\
.83 \\
.85 \\
.87\end{array}$ \\
\hline
\end{tabular}


TABLE 30 (Cont'd)

Salinity

Conversion from chlorinity to salinity $(\% / \infty)$

\begin{tabular}{|c|c|c|c|c|c|c|c|}
\hline $\mathrm{Cl}$ & $S$ & $\mathrm{Cl}$ & S & $\mathrm{Cl}$ & $S$ & $\mathrm{Cl}$ & S \\
\hline $\begin{array}{r}11.00 \\
.01 \\
.02 \\
.03 \\
.04 \\
.05 \\
.06 \\
.07 \\
.08 \\
.09\end{array}$ & $\begin{array}{r}19.89 \\
.90 \\
.92 \\
.94 \\
.96 \\
.98 \\
.99 \\
20.01 \\
.03 \\
.05\end{array}$ & $\begin{array}{r}11.40 \\
.41 \\
.42 \\
.43 \\
.44 \\
.45 \\
.46 \\
.47 \\
.48 \\
.49\end{array}$ & $\begin{array}{r}20.61 \\
.63 \\
.64 \\
.66 \\
.68 \\
.70 \\
.72 \\
.73 \\
.75 \\
.77\end{array}$ & $\begin{array}{r}11.80 \\
.81 \\
.82 \\
.83 \\
.84 \\
.85 \\
.86 \\
.87 \\
.88 \\
.89\end{array}$ & $\begin{array}{r}21.33 \\
.35 \\
.37 \\
.38 \\
.40 \\
.42 \\
.44 \\
.46 \\
.47 \\
.49\end{array}$ & $\begin{array}{r}12.20 \\
.21 \\
.22 \\
.23 \\
.34 \\
.25 \\
.26 \\
.27 \\
.28 \\
.29\end{array}$ & $\begin{array}{r}22.05 \\
.07 \\
.09 \\
.11 \\
.12 \\
.14 \\
.16 \\
.18 \\
.20 \\
.21\end{array}$ \\
\hline $\begin{array}{r}11.10 \\
.11 \\
.12 \\
.13 \\
.14 \\
.15 \\
.16 \\
.17 \\
.18 \\
.19\end{array}$ & $\begin{array}{r}20.07 \\
.08 \\
.10 \\
.12 \\
.14 \\
.16 \\
.17 \\
.19 \\
.21 \\
.23\end{array}$ & $\begin{array}{r}11.50 \\
.51 \\
.52 \\
.53 \\
.54 \\
.55 \\
.56 \\
.57 \\
.58 \\
.59\end{array}$ & $\begin{array}{r}20.79 \\
.81 \\
.82 \\
.84 \\
.86 \\
.88 \\
.90 \\
.91 \\
.93 \\
.95\end{array}$ & $\begin{array}{r}11.90 \\
.91 \\
.92 \\
.93 \\
.94 \\
.95 \\
.96 \\
.97 \\
.98 \\
.99\end{array}$ & $\begin{array}{r}21.51 \\
.53 \\
.55 \\
.56 \\
.58 \\
.60 \\
.62 \\
.64 \\
.65 \\
.67\end{array}$ & $\begin{array}{r}12.30 \\
.31 \\
.32 \\
.33 \\
.34 \\
.35 \\
.36 \\
.37 \\
.38 \\
.39\end{array}$ & $\begin{array}{r}22.23 \\
.25 \\
.27 \\
.29 \\
.30 \\
.32 \\
.34 \\
.36 \\
.38 \\
.39\end{array}$ \\
\hline $\begin{array}{r}11.20 \\
.21 \\
.22 \\
.23 \\
.24 \\
.25 \\
.26 \\
.27 \\
.28 \\
.29\end{array}$ & $\begin{array}{r}20.25 \\
.26 \\
.28 \\
.30 \\
.32 \\
.34 \\
.35 \\
.37 \\
.39 \\
.41\end{array}$ & $\begin{array}{r}11.60 \\
.61 \\
.62 \\
.63 \\
.64 \\
.65 \\
.66 \\
.67 \\
.68 \\
.69\end{array}$ & $\begin{array}{r}20.97 \\
.99 \\
21.00 \\
.02 \\
.04 \\
.06 \\
.08 \\
.09 \\
.11 \\
.13\end{array}$ & $\begin{array}{r}12.00 \\
.01 \\
.02 \\
.03 \\
.04 \\
.05 \\
.06 \\
.07 \\
.08 \\
.09\end{array}$ & $\begin{array}{r}21.69 \\
.71 \\
.73 \\
.74 \\
.76 \\
.78 \\
.80 \\
.82 \\
.83 \\
.85\end{array}$ & $\begin{array}{r}12.40 \\
.41 \\
.42 \\
.43 \\
.44 \\
.45 \\
.46 \\
.47 \\
.48 \\
.49\end{array}$ & $\begin{array}{r}22.41 \\
.43 \\
.45 \\
.47 \\
.48 \\
.50 \\
.52 \\
.54 \\
.56 \\
.57\end{array}$ \\
\hline $\begin{array}{r}11.30 \\
.31 \\
.32 \\
.33 \\
.34 \\
.35 \\
.36 \\
.37 \\
.38 \\
.39\end{array}$ & $\begin{array}{r}20.43 \\
.44 \\
.46 \\
.48 \\
.50 \\
.52 \\
.53 \\
.55 \\
.57 \\
.59\end{array}$ & $\begin{array}{r}11.70 \\
.71 \\
.72 \\
.73 \\
.74 \\
.75 \\
.76 \\
.77 \\
.78 \\
.79\end{array}$ & $\begin{array}{r}21.15 \\
.17 \\
.18 \\
.20 \\
.22 \\
.24 \\
.26 \\
.27 \\
.29 \\
.31\end{array}$ & $\begin{array}{r}12.10 \\
.11 \\
.12 \\
.13 \\
.14 \\
.15 \\
.16 \\
.17 \\
.18 \\
.19\end{array}$ & $\begin{array}{r}21.87 \\
.89 \\
.91 \\
.92 \\
.94 \\
.96 \\
.98 \\
22.00 \\
.01 \\
.03\end{array}$ & $\begin{array}{r}12.50 \\
.51 \\
.52 \\
.53 \\
.54 \\
.55 \\
.56 \\
.57 \\
.58 \\
.59\end{array}$ & $\begin{array}{r}22.59 \\
.61 \\
.63 \\
.65 \\
.66 \\
.68 \\
.70 \\
.72 \\
.74 \\
.75\end{array}$ \\
\hline
\end{tabular}


TABLE 30 (Cont'd)

Salinity

Conversion from chlorinity to salinity $(\%)$

\begin{tabular}{|c|c|c|c|c|c|c|c|}
\hline $\mathrm{Cl}$ & $S$ & $\mathrm{Cl}$ & $S$ & $\mathrm{Cl}$ & $S$ & $\mathrm{Cl}$ & $S$ \\
\hline $\begin{array}{r}12.60 \\
.61 \\
.62 \\
.63 \\
.64 \\
.65 \\
.66 \\
.67 \\
.68 \\
.69\end{array}$ & $\begin{array}{r}22.77 \\
.79 \\
.81 \\
.83 \\
.85 \\
.86 \\
.88 \\
.90 \\
.92 \\
.94\end{array}$ & $\begin{array}{r}13.00 \\
.01 \\
.02 \\
.03 \\
.04 \\
.05 \\
.06 \\
.07 \\
.08 \\
.09\end{array}$ & $\begin{array}{r}23.50 \\
.51 \\
.53 \\
.55 \\
.57 \\
.59 \\
.60 \\
.62 \\
.64 \\
.66\end{array}$ & $\begin{array}{r}13.40 \\
.41 \\
.42 \\
.43 \\
.44 \\
.45 \\
.46 \\
.47 \\
.48 \\
.49\end{array}$ & $\begin{array}{r}24.22 \\
.24 \\
.25 \\
.27 \\
.29 \\
.31 \\
.33 \\
.34 \\
.36 \\
.38\end{array}$ & $\begin{array}{r}13.80 \\
.81 \\
.82 \\
.83 \\
.84 \\
.85 \\
.86 \\
.87 \\
.88 \\
.89\end{array}$ & $\begin{array}{r}24.94 \\
.96 \\
.98 \\
.99 \\
25.01 \\
.03 \\
.05 \\
.07 \\
.08 \\
.10\end{array}$ \\
\hline $\begin{array}{r}12.70 \\
.71 \\
.72 \\
.73 \\
.74 \\
.75 \\
.76 \\
.77 \\
.78 \\
.79\end{array}$ & $\begin{array}{r}22.95 \\
.97 \\
.99 \\
23.01 \\
.03 \\
.04 \\
.06 \\
.08 \\
.10 \\
.12\end{array}$ & $\begin{array}{r}13.10 \\
.11 \\
.12 \\
.13 \\
.14 \\
.15 \\
.16 \\
.17 \\
.18 \\
.19\end{array}$ & $\begin{array}{r}23.68 \\
.69 \\
.71 \\
.73 \\
.75 \\
.77 \\
.78 \\
.80 \\
.82 \\
.84\end{array}$ & $\begin{array}{r}13.50 \\
.51 \\
.52 \\
.53 \\
.54 \\
.55 \\
.56 \\
.57 \\
.58 \\
.59\end{array}$ & $\begin{array}{r}24.40 \\
.42 \\
.43 \\
.45 \\
.47 \\
.49 \\
.51 \\
.52 \\
.54 \\
.56\end{array}$ & $\begin{array}{r}13.90 \\
.91 \\
.92 \\
.93 \\
.94 \\
.95 \\
.96 \\
.97 \\
.98 \\
.99\end{array}$ & $\begin{array}{r}25.12 \\
.14 \\
.16 \\
.17 \\
.19 \\
.21 \\
.23 \\
.25 \\
.26 \\
.28\end{array}$ \\
\hline $\begin{array}{r}12.80 \\
.81 \\
.82 \\
.83 \\
.84 \\
.85 \\
.86 \\
.87 \\
.88 \\
.89\end{array}$ & $\begin{array}{r}23.13 \\
.15 \\
.17 \\
.19 \\
.21 \\
.22 \\
.24 \\
.26 \\
.28 \\
.30\end{array}$ & $\begin{array}{r}13.20 \\
.21 \\
.22 \\
.23 \\
.24 \\
.25 \\
.26 \\
.27 \\
.28 \\
.29\end{array}$ & $\begin{array}{r}23.86 \\
.87 \\
.89 \\
.91 \\
.93 \\
.95 \\
.96 \\
.98 \\
24.00 \\
.02\end{array}$ & $\begin{array}{r}13.60 \\
.61 \\
.62 \\
.63 \\
.64 \\
.65 \\
.66 \\
.67 \\
.68 \\
.69\end{array}$ & $\begin{array}{r}24.58 \\
.60 \\
.61 \\
.63 \\
.65 \\
.67 \\
.69 \\
.70 \\
.72 \\
.74\end{array}$ & $\begin{array}{r}14.00 \\
.01 \\
.02 \\
.03 \\
.04 \\
.05 \\
.06 \\
.07 \\
.08 \\
.09\end{array}$ & $\begin{array}{r}25.30 \\
.32 \\
.34 \\
.35 \\
.37 \\
.39 \\
.41 \\
.43 \\
.44 \\
.46\end{array}$ \\
\hline $\begin{array}{r}12.90 \\
.91 \\
.92 \\
.93 \\
.94 \\
.95 \\
.96 \\
.97 \\
.98 \\
.99\end{array}$ & $\begin{array}{r}23.31 \\
.33 \\
.35 \\
.37 \\
.39 \\
.40 \\
.42 \\
.44 \\
.46 \\
.48\end{array}$ & $\begin{array}{r}13.30 \\
.31 \\
.32 \\
.33 \\
.34 \\
.35 \\
.36 \\
.37 \\
.38 \\
.39\end{array}$ & $\begin{array}{r}24.04 \\
.05 \\
.07 \\
.09 \\
.11 \\
.13 \\
.14 \\
.16 \\
.18 \\
.20\end{array}$ & $\begin{array}{r}13.70 \\
.71 \\
.72 \\
.73 \\
.74 \\
.75 \\
.76 \\
.77 \\
.78 \\
.79\end{array}$ & $\begin{array}{r}24.76 \\
.78 \\
.79 \\
.81 \\
.83 \\
.85 \\
.87 \\
.88 \\
.90 \\
.92\end{array}$ & $\begin{array}{r}14.10 \\
.11 \\
.12 \\
.13 \\
.14 \\
.15 \\
.16 \\
.17 \\
.18 \\
.19\end{array}$ & $\begin{array}{r}25.48 \\
.50 \\
.52 \\
.53 \\
.55 \\
.57 \\
.59 \\
.61 \\
.62 \\
.64\end{array}$ \\
\hline
\end{tabular}


TABLE 30 (Cont'd)

Salinfty

Conversion from chlorinity to salinity $(\%)$

\begin{tabular}{|c|c|c|c|c|c|c|c|}
\hline $\mathrm{Cl}$ & S & $\mathrm{Cl}$ & S & $\mathrm{Cl}$ & S & $\mathrm{Cl}$ & S \\
\hline $\begin{array}{r}14.20 \\
.21 \\
.22 \\
.23 \\
.24 \\
.25 \\
.26 \\
.27 \\
.28 \\
.29\end{array}$ & $\begin{array}{r}25.66 \\
.68 \\
.70 \\
.72 \\
.73 \\
.75 \\
.77 \\
.79 \\
.81 \\
.82\end{array}$ & $\begin{array}{r}14.60 \\
.61 \\
.62 \\
.63 \\
.64 \\
.65 \\
.66 \\
.67 \\
.68 \\
.69\end{array}$ & $\begin{array}{r}26.38 \\
.40 \\
.42 \\
.44 \\
.46 \\
.47 \\
.49 \\
.51 \\
.53 \\
.55\end{array}$ & $\begin{array}{r}15.00 \\
.01 \\
.02 \\
.03 \\
.04 \\
.05 \\
.06 \\
.07 \\
.08 \\
.09\end{array}$ & $\begin{array}{r}27.11 \\
.12 \\
.14 \\
.16 \\
.18 \\
.20 \\
.21 \\
.23 \\
.25 \\
.27\end{array}$ & $\begin{array}{r}15.40 \\
.41 \\
.42 \\
.43 \\
.44 \\
.45 \\
.46 \\
.47 \\
.48 \\
.49\end{array}$ & $\begin{array}{r}27.83 \\
.85 \\
.86 \\
.88 \\
.90 \\
.92 \\
.94 \\
.95 \\
.97 \\
.99\end{array}$ \\
\hline $\begin{array}{r}14.30 \\
.31 \\
.32 \\
.33 \\
.34 \\
.35 \\
.36 \\
.37 \\
.38 \\
.39\end{array}$ & $\begin{array}{r}25.84 \\
.86 \\
.88 \\
.90 \\
.91 \\
.93 \\
.95 \\
.97 \\
.99 \\
26.00\end{array}$ & $\begin{array}{r}14.70 \\
.71 \\
.72 \\
.73 \\
.74 \\
.75 \\
.76 \\
.77 \\
.78 \\
.79\end{array}$ & $\begin{array}{r}26.56 \\
.58 \\
.60 \\
.62 \\
.64 \\
.65 \\
.67 \\
.69 \\
.71 \\
.73\end{array}$ & $\begin{array}{r}15.10 \\
.11 \\
.12 \\
.13 \\
.14 \\
.15 \\
.16 \\
.17 \\
.18 \\
.19\end{array}$ & $\begin{array}{r}27.29 \\
.30 \\
.32 \\
.34 \\
.36 \\
.38 \\
.39 \\
.41 \\
.43 \\
.45\end{array}$ & $\begin{array}{r}15.50 \\
.51 \\
.52 \\
.53 \\
.54 \\
.55 \\
.56 \\
.57 \\
.58 \\
.59\end{array}$ & $\begin{array}{r}28.01 \\
.03 \\
.04 \\
.06 \\
.08 \\
.10 \\
.12 \\
.13 \\
.15 \\
.17\end{array}$ \\
\hline $\begin{array}{r}14.40 \\
.41 \\
.42 \\
.43 \\
.44 \\
.45 \\
.46 \\
.47 \\
.48 \\
.49\end{array}$ & $\begin{array}{r}26.02 \\
.04 \\
.06 \\
.08 \\
.09 \\
.11 \\
.13 \\
.15 \\
.17 \\
.18\end{array}$ & $\begin{array}{r}14.80 \\
.81 \\
.82 \\
.83 \\
.84 \\
.83 \\
.86 \\
.87 \\
.88 \\
.89\end{array}$ & $\begin{array}{r}26.74 \\
.76 \\
.78 \\
.80 \\
.82 \\
.83 \\
.85 \\
.87 \\
.89 \\
.91\end{array}$ & $\begin{array}{r}15.20 \\
.21 \\
.22 \\
.23 \\
.24 \\
.25 \\
.26 \\
.27 \\
.28 \\
.29\end{array}$ & $\begin{array}{r}27.47 \\
.48 \\
.50 \\
.52 \\
.54 \\
.56 \\
.57 \\
.59 \\
.61 \\
.63\end{array}$ & $\begin{array}{r}15.60 \\
.61 \\
.62 \\
.63 \\
.64 \\
.65 \\
.66 \\
.67 \\
.68 \\
.69\end{array}$ & $\begin{array}{r}28.19 \\
.21 \\
.22 \\
.24 \\
.26 \\
.28 \\
.30 \\
.31 \\
.33 \\
.35\end{array}$ \\
\hline $\begin{array}{r}14.50 \\
.51 \\
.52 \\
.53 \\
.54 \\
.55 \\
.56 \\
.57 \\
.58 \\
.59\end{array}$ & $\begin{array}{r}26.20 \\
.22 \\
.24 \\
.26 \\
.27 \\
.29 \\
.31 \\
.33 \\
.35 \\
.36\end{array}$ & $\begin{array}{r}14.90 \\
.91 \\
.92 \\
.93 \\
.94 \\
.95 \\
.96 \\
.97 \\
.98 \\
.99\end{array}$ & $\begin{array}{r}26.92 \\
.94 \\
.96 \\
.98 \\
27.00 \\
.01 \\
.03 \\
.05 \\
.07 \\
.09\end{array}$ & $\begin{array}{r}15.30 \\
.31 \\
.32 \\
.33 \\
.34 \\
.35 \\
.36 \\
.37 \\
.38 \\
.39\end{array}$ & $\begin{array}{r}27.65 \\
.66 \\
.68 \\
.70 \\
.72 \\
.74 \\
.75 \\
.77 \\
.79 \\
.81\end{array}$ & $\begin{array}{r}15.70 \\
.71 \\
.72 \\
.73 \\
.74 \\
.75 \\
.76 \\
.77 \\
.78 \\
.79\end{array}$ & $\begin{array}{r}28.37 \\
.39 \\
.40 \\
.42 \\
.44 \\
.46 \\
.48 \\
.49 \\
.51 \\
.53\end{array}$ \\
\hline
\end{tabular}


TABLE 30 (Cont'd)

Salinity

Conversion from chlorinity to salinity $(\%)$

\begin{tabular}{|c|c|c|c|c|c|c|c|}
\hline $\mathrm{Cl}$ & $S$ & $\mathrm{Cl}$ & S & $\mathrm{Cl}$ & $S$ & $\mathrm{Cl}$ & S \\
\hline $\begin{array}{r}15.80 \\
.81 \\
.82 \\
.83 \\
.84 \\
.85 \\
.86 \\
.87 \\
.88 \\
.89\end{array}$ & $\begin{array}{r}28.55 \\
.57 \\
.59 \\
.60 \\
.62 \\
.64 \\
.66 \\
.68 \\
.69 \\
.71\end{array}$ & $\begin{array}{r}16.20 \\
.21 \\
.22 \\
.23 \\
.24 \\
.25 \\
.26 \\
.27 \\
.28 \\
.29\end{array}$ & $\begin{array}{r}29.27 \\
.29 \\
.31 \\
.33 \\
.34 \\
.36 \\
.38 \\
.40 \\
.42 \\
.43\end{array}$ & $\begin{array}{r}16.60 \\
.61 \\
.62 \\
.63 \\
.64 \\
.65 \\
.66 \\
.67 \\
.68 \\
.69\end{array}$ & $\begin{array}{r}29.99 \\
30.01 \\
.03 \\
.05 \\
.07 \\
.08 \\
.10 \\
.12 \\
.14 \\
.16\end{array}$ & $\begin{array}{r}17.00 \\
.01 \\
.02 \\
.03 \\
.04 \\
.05 \\
.06 \\
.07 \\
.08 \\
.09\end{array}$ & $\begin{array}{r}30.72 \\
.73 \\
.75 \\
.77 \\
.79 \\
.81 \\
.82 \\
.84 \\
.86 \\
.88\end{array}$ \\
\hline $\begin{array}{r}15.90 \\
.91 \\
.92 \\
.93 \\
.94 \\
.95 \\
.96 \\
.97 \\
.98 \\
.99\end{array}$ & $\begin{array}{r}28.73 \\
.75 \\
.77 \\
.78 \\
.80 \\
.82 \\
.84 \\
.86 \\
.87 \\
.89\end{array}$ & $\begin{array}{r}16.30 \\
.31 \\
.32 \\
.33 \\
.34 \\
.35 \\
.36 \\
.37 \\
.38 \\
.39\end{array}$ & $\begin{array}{r}29.45 \\
.47 \\
.49 \\
.51 \\
.52 \\
.54 \\
.56 \\
.58 \\
.60 \\
.61\end{array}$ & $\begin{array}{r}16.70 \\
.71 \\
.72 \\
.73 \\
.74 \\
.75 \\
.76 \\
.77 \\
.78 \\
.79\end{array}$ & $\begin{array}{r}30.17 \\
.19 \\
.21 \\
.23 \\
.25 \\
.26 \\
.28 \\
.30 \\
.32 \\
.34\end{array}$ & $\begin{array}{r}17.10 \\
.11 \\
.12 \\
.13 \\
.14 \\
.15 \\
.16 \\
.17 \\
.18 \\
.19\end{array}$ & $\begin{array}{r}30.90 \\
.91 \\
.93 \\
.95 \\
.97 \\
.99 \\
31.00 \\
.02 \\
.04 \\
.06\end{array}$ \\
\hline $\begin{array}{r}16.00 \\
.01 \\
.02 \\
.03 \\
.04 \\
.05 \\
.06 \\
.07 \\
.08 \\
.09\end{array}$ & $\begin{array}{r}28.91 \\
.93 \\
.95 \\
.96 \\
.98 \\
29.00 \\
.02 \\
.04 \\
.05 \\
.07\end{array}$ & $\begin{array}{r}16.40 \\
.41 \\
.42 \\
.43 \\
.44 \\
.45 \\
.46 \\
.47 \\
.48 \\
.49\end{array}$ & $\begin{array}{r}29.63 \\
.65 \\
.67 \\
.69 \\
.70 \\
.72 \\
.74 \\
.76 \\
.78 \\
.79\end{array}$ & $\begin{array}{r}16.80 \\
.81 \\
.82 \\
.83 \\
.84 \\
.85 \\
.86 \\
.87 \\
.88 \\
.89\end{array}$ & $\begin{array}{r}30.35 \\
.37 \\
.39 \\
.41 \\
.43 \\
.44 \\
.46 \\
.48 \\
.50 \\
.52\end{array}$ & $\begin{array}{r}17.20 \\
.21 \\
.22 \\
.23 \\
.24 \\
.25 \\
.26 \\
.27 \\
.28 \\
.29\end{array}$ & $\begin{array}{r}31.08 \\
.09 \\
.11 \\
.13 \\
.15 \\
.17 \\
.18 \\
.20 \\
.22 \\
.24\end{array}$ \\
\hline $\begin{array}{r}16.10 \\
.11 \\
.12 \\
.13 \\
.14 \\
.15 \\
.16 \\
.17 \\
.18 \\
.19\end{array}$ & $\begin{array}{r}29.09 \\
.11 \\
.13 \\
.14 \\
.16 \\
.18 \\
.20 \\
.22 \\
.23 \\
.25\end{array}$ & $\begin{array}{r}16.50 \\
.51 \\
.52 \\
.53 \\
.54 \\
.55 \\
.56 \\
.57 \\
.58 \\
.59\end{array}$ & $\begin{array}{r}29.81 \\
.83 \\
.85 \\
.87 \\
.88 \\
.90 \\
.92 \\
.94 \\
.96 \\
.97\end{array}$ & $\begin{array}{r}16.90 \\
.91 \\
.92 \\
.93 \\
.94 \\
.95 \\
.96 \\
.97 \\
.98 \\
.99\end{array}$ & $\begin{array}{r}30.53 \\
.55 \\
.57 \\
.59 \\
.61 \\
.62 \\
.64 \\
.66 \\
.68 \\
.70\end{array}$ & $\begin{array}{r}17.30 \\
.31 \\
.32 \\
.33 \\
.34 \\
.35 \\
.36 \\
.37 \\
.38 \\
.39\end{array}$ & $\begin{array}{r}31.26 \\
.27 \\
.29 \\
.31 \\
.33 \\
.35 \\
.36 \\
.38 \\
.40 \\
.42\end{array}$ \\
\hline
\end{tabular}


TABLE 30 (Cont'd)

Salinity

Conversion from chlorinity to salinity $(\%)$

\begin{tabular}{|c|c|c|c|c|c|c|c|}
\hline $\mathrm{Cl}$ & $S$ & $\mathrm{Cl}$ & S & $\mathrm{Cl}$ & S & $\mathrm{Cl}$ & $S$ \\
\hline $\begin{array}{r}17.40 \\
.41 \\
.42 \\
.43 \\
.44 \\
.45 \\
.46 \\
.47 \\
.48 \\
.49\end{array}$ & $\begin{array}{r}31.44 \\
.46 \\
.47 \\
.49 \\
.51 \\
.53 \\
.55 \\
.56 \\
.58 \\
.60\end{array}$ & $\begin{array}{r}17.80 \\
.81 \\
.82 \\
.83 \\
.84 \\
.85 \\
.86 \\
.87 \\
.88 \\
.89\end{array}$ & $\begin{array}{r}32.16 \\
.18 \\
.20 \\
.21 \\
.23 \\
.25 \\
.27 \\
.29 \\
.30 \\
.32\end{array}$ & $\begin{array}{r}18.20 \\
.21 \\
.22 \\
.23 \\
.24 \\
.25 \\
.26 \\
.27 \\
.28 \\
.29\end{array}$ & $\begin{array}{r}32.88 \\
.90 \\
.92 \\
.94 \\
.95 \\
.97 \\
.99 \\
33.01 \\
.03 \\
.04\end{array}$ & $\begin{array}{r}18.60 \\
.61 \\
.62 \\
.63 \\
.64 \\
.65 \\
.66 \\
.67 \\
.68 \\
.69\end{array}$ & $\begin{array}{r}33.60 \\
.62 \\
.64 \\
.66 \\
.68 \\
.69 \\
.71 \\
.73 \\
.75 \\
.77\end{array}$ \\
\hline $\begin{array}{r}17.50 \\
.51 \\
.52 \\
.53 \\
.54 \\
.55 \\
.56 \\
.57 \\
.58 \\
.59\end{array}$ & $\begin{array}{r}31.62 \\
.64 \\
.65 \\
.67 \\
.69 \\
.71 \\
.73 \\
.74 \\
.76 \\
.78\end{array}$ & $\begin{array}{r}17.90 \\
.91 \\
.92 \\
.93 \\
.94 \\
.95 \\
.96 \\
.97 \\
.98 \\
.99\end{array}$ & $\begin{array}{r}32.34 \\
.36 \\
.38 \\
.39 \\
.41 \\
.43 \\
.45 \\
.47 \\
.48 \\
.50\end{array}$ & $\begin{array}{r}18.30 \\
.31 \\
.32 \\
.33 \\
.34 \\
.35 \\
.36 \\
.37 \\
.38 \\
.39\end{array}$ & $\begin{array}{r}33.06 \\
.08 \\
.10 \\
.12 \\
.13 \\
.15 \\
.17 \\
.19 \\
.21 \\
.22\end{array}$ & $\begin{array}{r}18.70 \\
.71 \\
.72 \\
.73 \\
.74 \\
.75 \\
.76 \\
.77 \\
.78 \\
.79\end{array}$ & $\begin{array}{r}33.78 \\
.80 \\
.82 \\
.84 \\
.86 \\
.87 \\
.89 \\
.91 \\
.93 \\
.95\end{array}$ \\
\hline $\begin{array}{r}17.60 \\
.61 \\
.62 \\
.63 \\
.64 \\
.65 \\
.66 \\
.67 \\
.68 \\
.69\end{array}$ & $\begin{array}{r}31.80 \\
.82 \\
.83 \\
.85 \\
.87 \\
.89 \\
.91 \\
.92 \\
.94 \\
.96\end{array}$ & $\begin{array}{r}18.00 \\
.01 \\
.02 \\
.03 \\
.04 \\
.05 \\
.06 \\
.07 \\
.08 \\
.09\end{array}$ & $\begin{array}{r}32.52 \\
.54 \\
.56 \\
.57 \\
.59 \\
.61 \\
.63 \\
.65 \\
.66 \\
.68\end{array}$ & $\begin{array}{r}18.40 \\
.41 \\
.42 \\
.43 \\
.44 \\
.45 \\
.46 \\
.47 \\
.48 \\
.49\end{array}$ & $\begin{array}{r}33.24 \\
.26 \\
.28 \\
.30 \\
.31 \\
.33 \\
.35 \\
.37 \\
.39 \\
.40\end{array}$ & $\begin{array}{r}18.80 \\
.81 \\
.82 \\
.83 \\
.84 \\
.85 \\
.86 \\
.87 \\
.88 \\
.89\end{array}$ & $\begin{array}{r}33.96 \\
.98 \\
34.00 \\
.02 \\
.04 \\
.05 \\
.07 \\
.09 \\
.11 \\
.13\end{array}$ \\
\hline $\begin{array}{r}17.70 \\
.71 \\
.72 \\
.73 \\
.74 \\
.75 \\
.76 \\
.77 \\
.78 \\
.79\end{array}$ & $\begin{array}{r}31.98 \\
32.00 \\
.01 \\
.03 \\
.05 \\
.07 \\
.09 \\
.10 \\
.12 \\
.14\end{array}$ & $\begin{array}{r}18.10 \\
.11 \\
.12 \\
.13 \\
.14 \\
.15 \\
.16 \\
.17 \\
.18 \\
.19\end{array}$ & $\begin{array}{r}32.70 \\
.72 \\
.74 \\
.75 \\
.77 \\
.79 \\
.81 \\
.83 \\
.84 \\
.86\end{array}$ & $\begin{array}{r}18.50 \\
.51 \\
.52 \\
.53 \\
.54 \\
.55 \\
.56 \\
.57 \\
.58 \\
.59\end{array}$ & $\begin{array}{r}33.42 \\
.44 \\
.46 \\
.48 \\
.49 \\
.51 \\
.53 \\
.55 \\
.57 \\
.58\end{array}$ & $\begin{array}{r}18.90 \\
.91 \\
.92 \\
.93 \\
.94 \\
.95 \\
.96 \\
.97 \\
.98 \\
.99\end{array}$ & $\begin{array}{r}34.14 \\
.16 \\
.18 \\
.20 \\
.22 \\
.23 \\
.25 \\
.27 \\
.29 \\
.31\end{array}$ \\
\hline
\end{tabular}


TABIE 30 (Cont' $\mathrm{d}$ )

Salinty

Conversion from chlorinity to salinity $(\%)$

\begin{tabular}{|c|c|c|c|c|c|c|c|}
\hline $\mathrm{Cl}$ & $S$ & $\mathrm{Cl}$ & S & $\mathrm{Cl}$ & S & $\mathrm{Cl}$ & $\mathrm{S}$ \\
\hline $\begin{array}{r}19.00 \\
.01 \\
.02 \\
.03 \\
.04 \\
.05 \\
.06 \\
.07 \\
.08 \\
.09\end{array}$ & $\begin{array}{r}34.33 \\
.34 \\
.36 \\
.38 \\
.40 \\
.42 \\
.43 \\
.45 \\
.47 \\
.49\end{array}$ & $\begin{array}{r}19.40 \\
.41 \\
.42 \\
.43 \\
.44 \\
.45 \\
.46 \\
.47 \\
.48 \\
.49\end{array}$ & $\begin{array}{r}35.05 \\
.07 \\
.08 \\
.10 \\
.12 \\
.14 \\
.16 \\
.17 \\
.19 \\
.21\end{array}$ & $\begin{array}{r}19.80 \\
.81 \\
.82 \\
.83 \\
.84 \\
.85 \\
.86 \\
.87 \\
.88 \\
.89\end{array}$ & $\begin{array}{r}35.77 \\
.79 \\
.81 \\
.82 \\
.84 \\
.86 \\
.88 \\
.90 \\
.91 \\
.93\end{array}$ & $\begin{array}{r}20.20 \\
.21 \\
.22 \\
.23 \\
.24 \\
.25 \\
.26 \\
.27 \\
.28 \\
.29\end{array}$ & $\begin{array}{r}36.49 \\
.51 \\
.53 \\
.55 \\
.56 \\
.58 \\
.60 \\
.62 \\
.64 \\
.65\end{array}$ \\
\hline $\begin{array}{r}19.10 \\
.11 \\
.12 \\
.13 \\
.14 \\
.15 \\
.16 \\
.17 \\
.18 \\
.19\end{array}$ & $\begin{array}{r}34.51 \\
.52 \\
.54 \\
.56 \\
.58 \\
.60 \\
.61 \\
.63 \\
.65 \\
.67\end{array}$ & $\begin{array}{r}19.50 \\
.51 \\
.52 \\
.53 \\
.54 \\
.55 \\
.56 \\
.57 \\
.58 \\
.59\end{array}$ & $\begin{array}{r}35.23 \\
.25 \\
.26 \\
.28 \\
.30 \\
.32 \\
.34 \\
.35 \\
.37 \\
.39\end{array}$ & $\begin{array}{r}19.90 \\
.91 \\
.92 \\
.93 \\
.94 \\
.95 \\
.96 \\
.97 \\
.98 \\
.99\end{array}$ & $\begin{array}{r}35.95 \\
.97 \\
.99 \\
36.00 \\
.02 \\
.04 \\
.06 \\
.08 \\
.09 \\
.011\end{array}$ & $\begin{array}{r}20.30 \\
.31 \\
.32 \\
.33 \\
.34 \\
.35 \\
.36 \\
.37 \\
.38 \\
.39\end{array}$ & $\begin{array}{r}36.67 \\
.69 \\
.71 \\
.73 \\
.74 \\
.76 \\
.78 \\
.80 \\
.82 \\
.83\end{array}$ \\
\hline $\begin{array}{r}19.20 \\
.21 \\
.22 \\
.23 \\
.24 \\
.25 \\
.26 \\
.27 \\
.28 \\
.29\end{array}$ & $\begin{array}{r}34.69 \\
.70 \\
.72 \\
.74 \\
.76 \\
.78 \\
.79 \\
.81 \\
.83 \\
.85\end{array}$ & $\begin{array}{r}19.60 \\
.61 \\
.62 \\
.63 \\
.64 \\
.65 \\
.66 \\
.67 \\
.68 \\
.69\end{array}$ & $\begin{array}{r}35.41 \\
.43 \\
.44 \\
.46 \\
.48 \\
.50 \\
.52 \\
.53 \\
.55 \\
.57\end{array}$ & $\begin{array}{r}20.00 \\
.01 \\
.02 \\
.03 \\
.04 \\
.05 \\
.06 \\
.07 \\
.08 \\
.09\end{array}$ & $\begin{array}{r}36.13 \\
.15 \\
.17 \\
.18 \\
.20 \\
.22 \\
.24 \\
.26 \\
.27 \\
.29\end{array}$ & $\begin{array}{r}20.40 \\
.41 \\
.42 \\
.43 \\
.44 \\
.45 \\
.46 \\
.47 \\
.48 \\
.49\end{array}$ & $\begin{array}{r}36.85 \\
.87 \\
.89 \\
.91 \\
.92 \\
.94 \\
.96 \\
.98 \\
37.00 \\
.01\end{array}$ \\
\hline $\begin{array}{r}19.30 \\
.31 \\
.32 \\
.33 \\
.34 \\
.35 \\
.36 \\
.37 \\
.38 \\
.39\end{array}$ & $\begin{array}{r}34.87 \\
.88 \\
.90 \\
.92 \\
.94 \\
.96 \\
.97 \\
.99 \\
35.01 \\
.03\end{array}$ & $\begin{array}{r}19.70 \\
.71 \\
.72 \\
.73 \\
.74 \\
.75 \\
.76 \\
.77 \\
.78 \\
.79\end{array}$ & $\begin{array}{r}35.59 \\
.61 \\
.62 \\
.64 \\
.66 \\
.68 \\
.70 \\
.71 \\
.73 \\
.75\end{array}$ & $\begin{array}{r}20.10 \\
.11 \\
.12 \\
.13 \\
.14 \\
.15 \\
.16 \\
.17 \\
.18 \\
.119\end{array}$ & $\begin{array}{r}36.31 \\
.33 \\
.35 \\
.36 \\
.38 \\
.40 \\
.42 \\
.44 \\
.45 \\
.47\end{array}$ & $\begin{array}{r}20.50 \\
.51 \\
.52 \\
.53 \\
.54 \\
.55 \\
.56 \\
.57 \\
.58 \\
.59\end{array}$ & $\begin{array}{r}37.03 \\
.05 \\
.07 \\
.09 \\
.10 \\
.12 \\
.14 \\
.16 \\
.18 \\
.19\end{array}$ \\
\hline
\end{tabular}


TABLE 30 (Cont'd)

Salinfty

Conversion from chlorinity to salinity $(\% / \infty)$

\begin{tabular}{|c|c|c|c|c|c|c|c|}
\hline $\mathrm{Cl}$ & $\mathrm{S}$ & $\mathrm{Cl}$ & S & $\mathrm{C}_{1}$ & $\mathrm{~S}$ & $\mathrm{Cl}$ & $\mathrm{S}$ \\
\hline $\begin{array}{r}20.60 \\
.61 \\
.62 \\
.63 \\
.64 \\
.65 \\
.66 \\
.67 \\
.68 \\
.69\end{array}$ & $\begin{array}{r}37.21 \\
.23 \\
.25 \\
.27 \\
.29 \\
.30 \\
.32 \\
.34 \\
.36 \\
.38\end{array}$ & $\begin{array}{r}21.00 \\
.01 \\
.02 \\
.03 \\
.04 \\
.05 \\
.06 \\
.07 \\
.08 \\
.09\end{array}$ & $\begin{array}{r}37.94 \\
.95 \\
.97 \\
.99 \\
38.01 \\
.03 \\
.04 \\
.06 \\
.08 \\
.10\end{array}$ & $\begin{array}{r}21.40 \\
.41 \\
.42 \\
.43 \\
.44 \\
.45 \\
.46 \\
.47 \\
.48 \\
.49\end{array}$ & $\begin{array}{r}38.66 \\
.68 \\
.69 \\
.71 \\
.73 \\
.75 \\
.77 \\
.78 \\
.80 \\
.82\end{array}$ & $\begin{array}{r}21.80 \\
.81 \\
.82 \\
.83 \\
.84 \\
.85 \\
.86 \\
.87 \\
.88 \\
.89\end{array}$ & $\begin{array}{r}39.38 \\
.40 \\
.42 \\
.43 \\
.45 \\
.47 \\
.49 \\
.51 \\
.52 \\
.54\end{array}$ \\
\hline $\begin{array}{r}20.70 \\
.71 \\
.72 \\
.73 \\
.74 \\
.75 \\
.76 \\
.77 \\
.78 \\
.79\end{array}$ & $\begin{array}{r}37.39 \\
.41 \\
.43 \\
.45 \\
.47 \\
.48 \\
.50 \\
.52 \\
.54 \\
.56\end{array}$ & $\begin{array}{r}21.10 \\
.11 \\
.12 \\
.13 \\
.14 \\
.15 \\
.16 \\
.17 \\
.18 \\
.19\end{array}$ & $\begin{array}{r}38.12 \\
.13 \\
.15 \\
.17 \\
.19 \\
.21 \\
.22 \\
.24 \\
.26 \\
.28\end{array}$ & $\begin{array}{r}21.50 \\
.51 \\
.52 \\
.53 \\
.54 \\
.55 \\
.56 \\
.57 \\
.58 \\
.59\end{array}$ & $\begin{array}{r}38.84 \\
.86 \\
.87 \\
.89 \\
.91 \\
.93 \\
.95 \\
.96 \\
.98 \\
39.00\end{array}$ & $\begin{array}{r}21.90 \\
.91 \\
.92 \\
.93 \\
.94 \\
.95 \\
.96 \\
.97 \\
.98 \\
.99\end{array}$ & $\begin{array}{r}39.56 \\
.58 \\
.60 \\
.61 \\
.63 \\
.65 \\
.67 \\
.69 \\
.70 \\
.72\end{array}$ \\
\hline $\begin{array}{r}20.80 \\
.81 \\
.82 \\
.83 \\
.84 \\
.85 \\
.86 \\
.87 \\
.88 \\
.89\end{array}$ & $\begin{array}{r}37.57 \\
.59 \\
.61 \\
.63 \\
.65 \\
.66 \\
.68 \\
.70 \\
.72 \\
.74\end{array}$ & $\begin{array}{r}21.20 \\
.21 \\
.22 \\
.23 \\
.24 \\
.25 \\
.26 \\
.27 \\
.28 \\
.29\end{array}$ & $\begin{array}{r}38.30 \\
.31 \\
.33 \\
.35 \\
.37 \\
.39 \\
.40 \\
.42 \\
.44 \\
.46\end{array}$ & $\begin{array}{r}21.60 \\
.61 \\
.62 \\
.63 \\
.64 \\
.65 \\
.66 \\
.67 \\
.68 \\
.69\end{array}$ & $\begin{array}{r}39.02 \\
.04 \\
.05 \\
.07 \\
.09 \\
.11 \\
.13 \\
.14 \\
.16 \\
.18\end{array}$ & $\begin{array}{r}22.00 \\
.01 \\
.02 \\
.03 \\
.04 \\
.05 \\
.06 \\
.07 \\
.08 \\
.09\end{array}$ & $\begin{array}{r}39.74 \\
.76 \\
.78 \\
.79 \\
.81 \\
.83 \\
.85 \\
.87 \\
.88 \\
.90\end{array}$ \\
\hline $\begin{array}{r}20.90 \\
.91 \\
.92 \\
.93 \\
.94 \\
.95 \\
.96 \\
.97 \\
.98 \\
.99\end{array}$ & $\begin{array}{r}37.75 \\
.77 \\
.79 \\
.81 \\
.83 \\
.84 \\
.86 \\
.88 \\
.90 \\
.92\end{array}$ & $\begin{array}{r}21.30 \\
.31 \\
.32 \\
.33 \\
.34 \\
.35 \\
.36 \\
.37 \\
.38 \\
.39\end{array}$ & $\begin{array}{r}38.48 \\
.49 \\
.51 \\
.53 \\
.55 \\
.57 \\
.58 \\
.60 \\
.62 \\
.64\end{array}$ & $\begin{array}{r}21.70 \\
.71 \\
.72 \\
.73 \\
.74 \\
.75 \\
.76 \\
.77 \\
.78 \\
.79\end{array}$ & $\begin{array}{r}39.20 \\
.22 \\
.23 \\
.25 \\
.27 \\
.29 \\
.31 \\
.32 \\
.34 \\
.36\end{array}$ & $\begin{array}{r}22.10 \\
.11 \\
.12 \\
.13 \\
.14 \\
.15 \\
.16 \\
.17 \\
.18 \\
.19\end{array}$ & $\begin{array}{r}39.92 \\
.94 \\
.96 \\
.97 \\
.99 \\
40.01 \\
.03 \\
.05 \\
.06 \\
.08\end{array}$ \\
\hline
\end{tabular}


TABLE 30 (Cont'd)

Salinity

Conversion from chlorinity to salinity $(\%)$

\begin{tabular}{|c|c|c|c|}
\hline $\mathrm{Cl}$ & S & $\mathrm{Cl}$ & S \\
\hline $\begin{array}{r}22.20 \\
.21 \\
.22 \\
.23 \\
.24 \\
.25 \\
.26 \\
.27 \\
.28 \\
.29\end{array}$ & $\begin{array}{r}40.10 \\
.12 \\
.14 \\
.16 \\
.17 \\
.19 \\
.21 \\
.23 \\
.25 \\
.26\end{array}$ & $\begin{array}{r}22.60 \\
.61 \\
.62 \\
.63 \\
.64 \\
.65 \\
.66 \\
.67 \\
.68 \\
.69\end{array}$ & $\begin{array}{r}40.82 \\
.84 \\
.86 \\
.88 \\
.90 \\
.91 \\
.93 \\
.95 \\
.97 \\
.99\end{array}$ \\
\hline $\begin{array}{r}22.30 \\
.31 \\
.32 \\
.33 \\
.34 \\
.35 \\
.36 \\
.37 \\
.38 \\
.39\end{array}$ & $\begin{array}{r}40.28 \\
.30 \\
.32 \\
.34 \\
.35 \\
.37 \\
.39 \\
.41 \\
.43 \\
.44\end{array}$ & $\begin{array}{r}22.70 \\
.71 \\
.72 \\
.73 \\
.74 \\
.75 \\
.76 \\
.77 \\
.78 \\
.79\end{array}$ & $\begin{array}{r}41.00 \\
.02 \\
.04 \\
.06 \\
.08 \\
.09 \\
.11 \\
.13 \\
.15 \\
.17\end{array}$ \\
\hline $\begin{array}{r}22.40 \\
.41 \\
.42 \\
.43 \\
.44 \\
.45 \\
.46 \\
.47 \\
.48 \\
.49\end{array}$ & $\begin{array}{r}40.46 \\
.48 \\
.50 \\
.52 \\
.53 \\
.55 \\
.57 \\
.59 \\
.61 \\
.62\end{array}$ & $\begin{array}{r}22.80 \\
.81 \\
.82 \\
.83 \\
.84 \\
.85 \\
.86 \\
.87 \\
.88 \\
.89\end{array}$ & $\begin{array}{r}41.18 \\
.20 \\
.22 \\
.24 \\
.26 \\
.27 \\
.29 \\
.31 \\
.33 \\
.35\end{array}$ \\
\hline $\begin{array}{r}22.50 \\
.51 \\
.52 \\
.53 \\
.54 \\
.55 \\
.56 \\
.57 \\
.58 \\
.59\end{array}$ & $\begin{array}{r}40.64 \\
.66 \\
.68 \\
.70 \\
.71 \\
.73 \\
.75 \\
.77 \\
.79 \\
.80\end{array}$ & $\begin{array}{r}22.90 \\
.91 \\
.92 \\
.93 \\
.94 \\
.95 \\
.96 \\
.97 \\
.98 \\
.99 \\
23.00\end{array}$ & $\begin{array}{r}41.36 \\
.38 \\
.40 \\
.42 \\
.44 \\
.45 \\
.47 \\
.49 \\
.51 \\
.53 \\
41.55\end{array}$ \\
\hline
\end{tabular}


TABIE 31

Oxygen

Conversion from milligrams per liter to milliliters per liter (NIP) $(1 \mathrm{mg} / \mathrm{l}=0.6998 \mathrm{ml} / \mathrm{l}$ )

\begin{tabular}{|c|c|c|c|c|c|c|c|c|c|c|}
\hline $\begin{array}{l}\text { Milligrams } \\
\text { per } \\
\text { Liter of } \mathrm{O}_{2}\end{array}$ & .00 & .01 & .02 & .03 & .04 & .05 & .06 & .07 & .08 & .09 \\
\hline $\begin{array}{l}0.0 \\
0.1 \\
0.2 \\
0.3 \\
0.4 \\
0.5\end{array}$ & $\begin{array}{l}0.00 \\
0.07 \\
0.14 \\
0.21 \\
0.28 \\
0.35\end{array}$ & $\begin{array}{l}0.01 \\
0.08 \\
0.15 \\
0.22 \\
0.29 \\
0.36\end{array}$ & $\begin{array}{l}0.01 \\
0.08 \\
0.15 \\
0.22 \\
0.29 \\
0.36\end{array}$ & $\begin{array}{l}0.02 \\
0.09 \\
0.16 \\
0.23 \\
0.30 \\
0.37\end{array}$ & $\begin{array}{l}0.03 \\
0.10 \\
0.17 \\
0.24 \\
0.31 \\
0.38\end{array}$ & $\begin{array}{l}0.03 \\
0.10 \\
0.17 \\
0.24 \\
0.31 \\
0.38\end{array}$ & $\begin{array}{l}0.04 \\
0.11 \\
0.18 \\
0.25 \\
0.32 \\
0.39\end{array}$ & $\begin{array}{l}0.05 \\
0.12 \\
0.19 \\
0.26 \\
0.33 \\
0.40\end{array}$ & $\begin{array}{l}0.06 \\
0.13 \\
0.20 \\
0.27 \\
0.34 \\
0.41\end{array}$ & $\begin{array}{l}0.06 \\
0.13 \\
0.20 \\
0.27 \\
0.34 \\
0.41\end{array}$ \\
\hline $\begin{array}{l}0.6 \\
0.7 \\
0.8 \\
0.9\end{array}$ & $\begin{array}{l}0.42 \\
0.49 \\
0.56 \\
0.63\end{array}$ & $\begin{array}{l}0.43 \\
0.50 \\
0.57 \\
0.64\end{array}$ & $\begin{array}{l}0.43 \\
0.50 \\
0.57 \\
0.64\end{array}$ & $\begin{array}{l}0.44 \\
0.51 \\
0.58 \\
0.65\end{array}$ & $\begin{array}{l}0.45 \\
0.52 \\
0.59 \\
0.66\end{array}$ & $\begin{array}{l}0.45 \\
0.52 \\
0.59 \\
0.66\end{array}$ & $\begin{array}{l}0.46 \\
0.53 \\
0.60 \\
0.67\end{array}$ & $\begin{array}{l}0.47 \\
0.54 \\
0.61 \\
0.68\end{array}$ & $\begin{array}{l}0.48 \\
0.55 \\
0.62 \\
0.69\end{array}$ & $\begin{array}{l}0.48 \\
0.55 \\
0.62 \\
0.69\end{array}$ \\
\hline
\end{tabular}

\begin{tabular}{|c|c|c|c|}
\hline mflligrams/liter & mill1liters/liter & mllligrams/liter & milliliters/liter \\
\hline $\begin{array}{r}1.0 \\
2.0 \\
3.0 \\
4.0 \\
5.0 \\
6.0 \\
7.0 \\
8.0 \\
9.0 \\
10.0 \\
11.0\end{array}$ & $\begin{array}{l}0.70 \\
1.40 \\
2.10 \\
2.80 \\
3.50 \\
4.20 \\
4.90 \\
5.60 \\
6.30 \\
7.00 \\
7.70\end{array}$ & $\begin{array}{l}12.0 \\
13.0 \\
14.0 \\
15.0 \\
16.0 \\
17.0 \\
18.0 \\
19.0 \\
20.0 \\
21.0 \\
22.0\end{array}$ & $\begin{array}{r}8.40 \\
9.10 \\
9.80 \\
10.50 \\
11.20 \\
11.90 \\
12.60 \\
13.30 \\
14.00 \\
14.70 \\
15.40\end{array}$ \\
\hline
\end{tabular}

Example: Convert 5.65 milligrams/liter of $\mathrm{O}_{2}$ to milliliters/liter.

$5.00 \mathrm{mill}$ igrams/liter $=3.50$

$0.65 \mathrm{milligrams} /$ liter $=0.45$

3.95 milliliters/liter (ans.) 
TABLE 32

Oxygen

Conversion from miliigram-atoms per liter to milliliters per liter ( 1 milligram-atom per liter of $\mathrm{O}_{2}=11.196$ milliliters per liter of $\mathrm{O}_{2}$ )

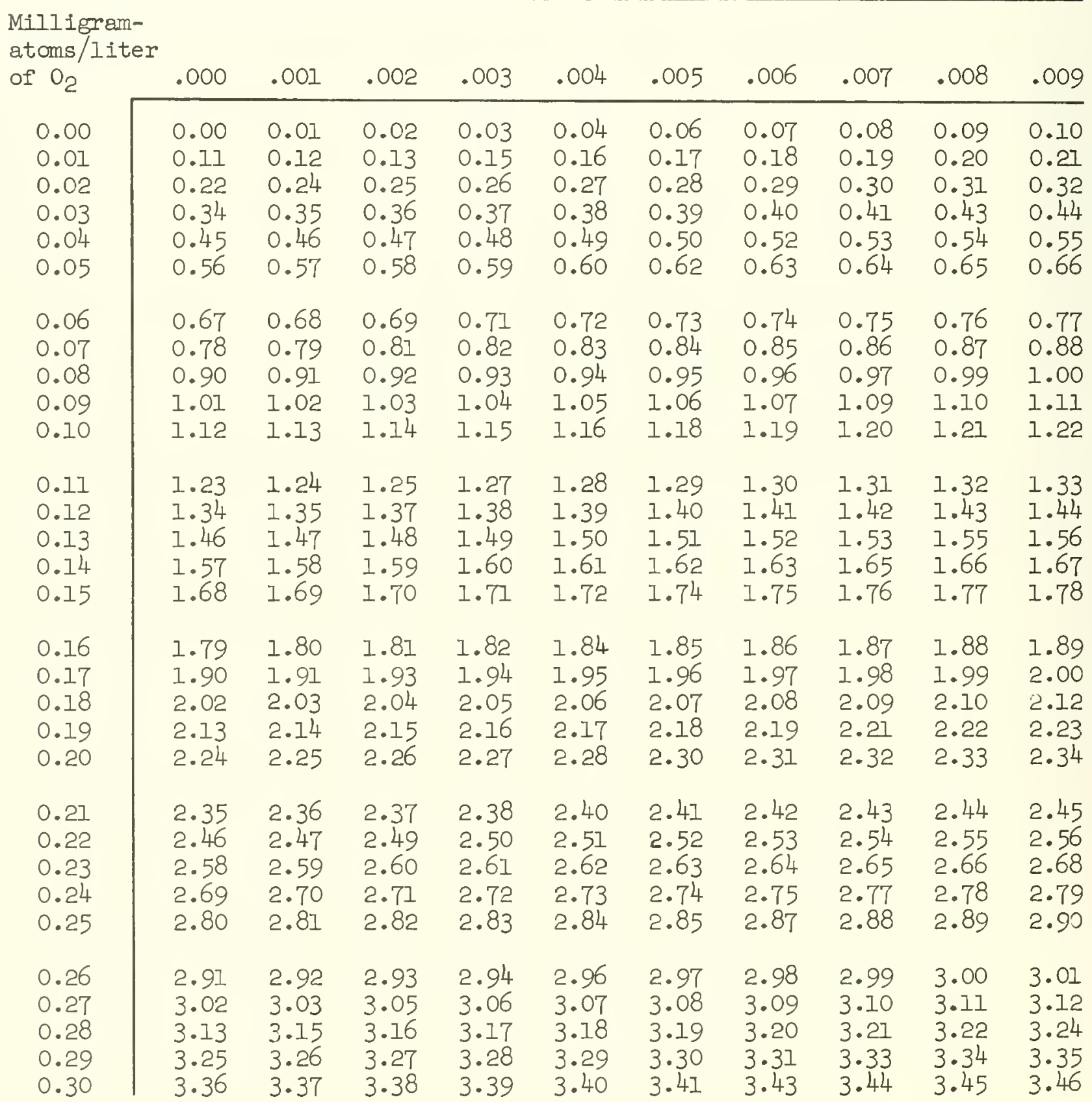


TABLE 32 (Cont'd)

Oxygen

Conversion from milligram-atoms per liter to milliliters per liter ( 1 milligram-atom per liter of $\mathrm{O}_{2}=11.196$ miliiliters per liter of $\mathrm{O}_{2}$ )

\begin{tabular}{|c|c|c|c|c|c|c|c|c|c|c|}
\hline $\begin{array}{l}\text { Milligl } \\
\text { atoms/J } \\
\text { of } \mathrm{O}_{2}\end{array}$ & .000 & .001 & .002 & .003 & .004 & .005 & .006 & .007 & .008 & .009 \\
\hline $\begin{array}{l}0.31 \\
0.32 \\
0.33 \\
0.34 \\
0.35\end{array}$ & $\begin{array}{l}3.47 \\
3.58 \\
3.69 \\
3.81 \\
3.92\end{array}$ & $\begin{array}{l}3.48 \\
3.59 \\
3.71 \\
3.82 \\
3.93\end{array}$ & $\begin{array}{l}3.49 \\
3.61 \\
3.72 \\
3.83 \\
3.94\end{array}$ & $\begin{array}{l}3.50 \\
3.62 \\
3.73 \\
3.84 \\
3.95\end{array}$ & $\begin{array}{l}3.52 \\
3.63 \\
3.74 \\
3.85 \\
3.96\end{array}$ & $\begin{array}{l}3.53 \\
3.64 \\
3.75 \\
3.86 \\
3.97\end{array}$ & $\begin{array}{l}3.54 \\
3.65 \\
3.76 \\
3.87 \\
3.99\end{array}$ & $\begin{array}{l}3.55 \\
3.66 \\
3.77 \\
3.89 \\
4.00\end{array}$ & $\begin{array}{l}3.56 \\
3.67 \\
3.78 \\
3.90 \\
4.01\end{array}$ & $\begin{array}{l}3.57 \\
3.68 \\
3.80 \\
3.91 \\
4.02\end{array}$ \\
\hline $\begin{array}{l}0.36 \\
0.37 \\
0.38 \\
0.39 \\
0.40\end{array}$ & $\begin{array}{l}4.03 \\
4.14 \\
4.25 \\
4.37 \\
4.48\end{array}$ & $\begin{array}{l}4.04 \\
4.15 \\
4.27 \\
4.38 \\
4.49\end{array}$ & $\begin{array}{l}4.05 \\
4.16 \\
4.28 \\
4.39 \\
4.50\end{array}$ & $\begin{array}{l}4.06 \\
4.18 \\
4.29 \\
4.40 \\
4.51\end{array}$ & $\begin{array}{l}4.08 \\
4.19 \\
4.30 \\
4.41 \\
4.52\end{array}$ & $\begin{array}{l}4.09 \\
4.20 \\
4.31 \\
4.42 \\
4.53\end{array}$ & $\begin{array}{l}4.10 \\
4.21 \\
4.32 \\
4.43 \\
4.55\end{array}$ & $\begin{array}{l}4.11 \\
4.22 \\
4.33 \\
4.44 \\
4.56\end{array}$ & $\begin{array}{l}4.12 \\
4.23 \\
4.34 \\
4.46 \\
4.57\end{array}$ & $\begin{array}{l}4.13 \\
4.24 \\
4.36 \\
4.47 \\
4.58\end{array}$ \\
\hline $\begin{array}{l}0.41 \\
0.42 \\
0.43 \\
0.44 \\
0.45\end{array}$ & $\begin{array}{l}4.59 \\
4.70 \\
4.81 \\
4.93 \\
5.04\end{array}$ & $\begin{array}{l}4.60 \\
4.71 \\
4.83 \\
4.94 \\
5.05\end{array}$ & $\begin{array}{l}4.61 \\
4.72 \\
4.84 \\
4.95 \\
5.06\end{array}$ & $\begin{array}{l}4.62 \\
4.74 \\
4.85 \\
4.96 \\
5.07\end{array}$ & $\begin{array}{l}4.64 \\
4.75 \\
4.86 \\
4.97 \\
5.08\end{array}$ & $\begin{array}{l}4.65 \\
4.76 \\
4.87 \\
4.98 \\
5.09\end{array}$ & $\begin{array}{l}4.66 \\
4.77 \\
4.88 \\
4.99 \\
5.11\end{array}$ & $\begin{array}{l}4.67 \\
4.78 \\
4.89 \\
5.00 \\
5.12\end{array}$ & $\begin{array}{l}4.68 \\
4.79 \\
4.90 \\
5.02 \\
5.13\end{array}$ & $\begin{array}{l}4.69 \\
4.80 \\
4.92 \\
5.03 \\
5.14\end{array}$ \\
\hline $\begin{array}{l}0.46 \\
0.47 \\
0.48 \\
0.49 \\
0.50\end{array}$ & $\begin{array}{l}5.15 \\
5.26 \\
5.37 \\
5.49 \\
5.60\end{array}$ & $\begin{array}{l}5.16 \\
5.27 \\
5.39 \\
5.50 \\
5.61\end{array}$ & $\begin{array}{l}5.17 \\
5.28 \\
5.40 \\
5.51 \\
5.62\end{array}$ & $\begin{array}{l}5.18 \\
5.30 \\
5.41 \\
5.52 \\
5.63\end{array}$ & $\begin{array}{l}5.19 \\
5.31 \\
5.42 \\
5.53 \\
5.64\end{array}$ & $\begin{array}{l}5.21 \\
5.32 \\
5.43 \\
5.54 \\
5.65\end{array}$ & $\begin{array}{l}5.22 \\
5.33 \\
5.44 \\
5.55 \\
5.67\end{array}$ & $\begin{array}{l}5.23 \\
5.34 \\
5.45 \\
5.56 \\
5.68\end{array}$ & $\begin{array}{l}5.24 \\
5.35 \\
5.46 \\
5.58 \\
5.69\end{array}$ & $\begin{array}{l}5.25 \\
5.36 \\
5.47 \\
5.59 \\
5.70\end{array}$ \\
\hline $\begin{array}{l}0.51 \\
0.52 \\
0.53 \\
0.54 \\
0.55\end{array}$ & $\begin{array}{l}5.71 \\
5.82 \\
5.93 \\
6.05 \\
6.16\end{array}$ & $\begin{array}{l}5.72 \\
5.83 \\
5.95 \\
6.06 \\
6.17\end{array}$ & $\begin{array}{l}5.73 \\
5.84 \\
5.96 \\
6.07 \\
6.18\end{array}$ & $\begin{array}{l}5.74 \\
5.86 \\
5.97 \\
6.08 \\
6.19\end{array}$ & $\begin{array}{l}5.75 \\
5.87 \\
5.98 \\
6.09 \\
6.20\end{array}$ & $\begin{array}{l}5.77 \\
5.88 \\
5.99 \\
6.10 \\
6.21\end{array}$ & $\begin{array}{l}5.78 \\
5.89 \\
6.00 \\
6.11 \\
6.22\end{array}$ & $\begin{array}{l}5.79 \\
5.90 \\
6.01 \\
6.12 \\
6.24\end{array}$ & $\begin{array}{l}5.80 \\
5.91 \\
6.02 \\
6.14 \\
6.25\end{array}$ & $\begin{array}{l}5.81 \\
5.92 \\
6.03 \\
6.15 \\
6.26\end{array}$ \\
\hline $\begin{array}{l}0.56 \\
0.57 \\
0.58 \\
0.59 \\
0.60\end{array}$ & $\begin{array}{l}6.27 \\
6.38 \\
6.49 \\
6.61 \\
6.72\end{array}$ & $\begin{array}{l}6.28 \\
6.39 \\
6.50 \\
6.62 \\
6.73\end{array}$ & $\begin{array}{l}6.29 \\
6.40 \\
6.52 \\
6.63 \\
6.74\end{array}$ & $\begin{array}{l}6.30 \\
6.42 \\
6.53 \\
6.64 \\
6.75\end{array}$ & $\begin{array}{l}6.31 \\
6.43 \\
6.54 \\
6.65 \\
6.76\end{array}$ & $\begin{array}{l}6.33 \\
6.44 \\
6.55 \\
6.66 \\
6.77\end{array}$ & $\begin{array}{l}6.34 \\
6.45 \\
6.56 \\
6.67 \\
6.78\end{array}$ & $\begin{array}{l}6.35 \\
6.46 \\
6.57 \\
6.68 \\
6.80\end{array}$ & $\begin{array}{l}6.36 \\
6.47 \\
6.58 \\
6.70 \\
6.81\end{array}$ & $\begin{array}{l}6.37 \\
6.48 \\
6.59 \\
6.71 \\
6.82\end{array}$ \\
\hline
\end{tabular}




\section{TABLE 32 (Cont'd)}

\section{Oxygen}

Conversion from mllligram-atoms per liter to milliliters per liter ( 1 milligram-atom per liter of $\mathrm{O}_{2}=11.196$ milliliters per liter of $\mathrm{O}_{2}$ )

Milligram-

atoms/liter

of $\mathrm{O}_{2}$

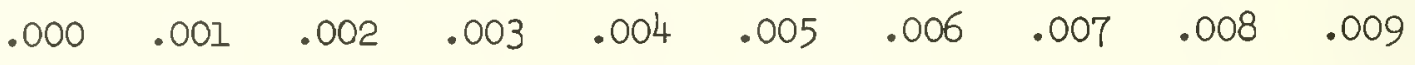

\begin{tabular}{|c|c|c|c|c|c|c|c|c|c|c|}
\hline $\begin{array}{l}0.61 \\
0.62 \\
0.63 \\
0.64 \\
0.65\end{array}$ & $\begin{array}{l}6.83 \\
6.94 \\
7.05 \\
7.17 \\
7.28\end{array}$ & $\begin{array}{l}6.84 \\
6.95 \\
7.06 \\
7.18 \\
7.29\end{array}$ & $\begin{array}{l}6.85 \\
6.96 \\
7.08 \\
7.19 \\
7.30\end{array}$ & $\begin{array}{l}6.86 \\
6.98 \\
7.09 \\
7.20 \\
7.31\end{array}$ & $\begin{array}{l}6.87 \\
6.99 \\
7.10 \\
7.21 \\
7.32\end{array}$ & $\begin{array}{l}6.89 \\
7.00 \\
7.11 \\
7.22 \\
7.33\end{array}$ & $\begin{array}{l}6.90 \\
7.01 \\
7.12 \\
7.23 \\
7.34\end{array}$ & $\begin{array}{l}6.91 \\
7.02 \\
7.13 \\
7.24 \\
7.36\end{array}$ & $\begin{array}{l}6.92 \\
7.03 \\
7.14 \\
7.26 \\
7.37\end{array}$ & $\begin{array}{l}6.93 \\
7.04 \\
7.15 \\
7.27 \\
7.38\end{array}$ \\
\hline $\begin{array}{l}.66 \\
.67 \\
.68 \\
.69 \\
.70\end{array}$ & $\begin{array}{l}7.39 \\
7.50 \\
7.61 \\
7.73 \\
7.84\end{array}$ & $\begin{array}{l}7.40 \\
7.51 \\
7.62 \\
7.74 \\
7.85\end{array}$ & $\begin{array}{l}7.41 \\
7.52 \\
7.64 \\
7.75 \\
7.86\end{array}$ & $\begin{array}{l}7.42 \\
7.53 \\
7.65 \\
7.76 \\
7.87\end{array}$ & $\begin{array}{l}7.43 \\
7.55 \\
7.66 \\
7.77 \\
7.88\end{array}$ & $\begin{array}{l}7.45 \\
7.56 \\
7.67 \\
7.78 \\
7.89\end{array}$ & $\begin{array}{l}7.46 \\
7.57 \\
7.68 \\
7.79 \\
7.90\end{array}$ & $\begin{array}{l}7.47 \\
7.58 \\
7.69 \\
7.80 \\
7.92\end{array}$ & $\begin{array}{l}7.48 \\
7.59 \\
7.70 \\
7.81 \\
7.93\end{array}$ & $\begin{array}{l}7.8 \\
7.9\end{array}$ \\
\hline $\begin{array}{l}.71 \\
.72 \\
.73 \\
.74 \\
.75\end{array}$ & $\begin{array}{l}7.95 \\
8.06 \\
8.17 \\
8.29 \\
8.40\end{array}$ & $\begin{array}{l}7.96 \\
8.07 \\
8.18 \\
8.30 \\
8.41\end{array}$ & $\begin{array}{l}7.97 \\
8.08 \\
8.20 \\
8.31 \\
8.42\end{array}$ & $\begin{array}{l}7.98 \\
8.09 \\
8.21 \\
8.32 \\
8.43\end{array}$ & $\begin{array}{l}7.99 \\
8.11 \\
8.22 \\
8.33 \\
8.44\end{array}$ & $\begin{array}{l}8.01 \\
8.12 \\
8.23 \\
8.34 \\
8.45\end{array}$ & $\begin{array}{l}8.02 \\
8.13 \\
8.24 \\
8.35 \\
8.46\end{array}$ & $\begin{array}{l}8.03 \\
8.14 \\
8.25 \\
8.36 \\
8.48\end{array}$ & $\begin{array}{l}8.04 \\
8.15 \\
8.26 \\
8.37 \\
8.49\end{array}$ & 8. \\
\hline $\begin{array}{l}.78 \\
.79 \\
.80\end{array}$ & $\begin{array}{l}8.51 \\
8.62 \\
8.73 \\
8.84 \\
8.96\end{array}$ & $\begin{array}{l}8.52 \\
8.63 \\
8.74 \\
8.86 \\
8.97\end{array}$ & $\begin{array}{l}8.53 \\
8.64 \\
8.76 \\
8.87 \\
8.98\end{array}$ & $\begin{array}{l}8.54 \\
8.65 \\
8.77 \\
8.88 \\
8.99\end{array}$ & $\begin{array}{l}8.55 \\
8.67 \\
8.78 \\
8.89 \\
9.00\end{array}$ & $\begin{array}{l}8.56 \\
8.68 \\
8.79 \\
8.90 \\
9.01\end{array}$ & $\begin{array}{l}8.58 \\
8.69 \\
8.80 \\
8.91 \\
9.02\end{array}$ & $\begin{array}{l}8.59 \\
8.70 \\
8.81 \\
8.92 \\
9.04\end{array}$ & $\begin{array}{l}8.60 \\
8.71 \\
8.82 \\
8.93 \\
9.05\end{array}$ & $\begin{array}{l}8 . \\
8 . \\
9 .\end{array}$ \\
\hline $\begin{array}{l}0.82 \\
0.83 \\
0.84 \\
0.85\end{array}$ & $\begin{array}{l}9.07 \\
9.18 \\
9.29 \\
9.40 \\
9.52\end{array}$ & $\begin{array}{l}9.08 \\
9.19 \\
9.30 \\
9.42 \\
9.53\end{array}$ & $\begin{array}{l}9.09 \\
9.20 \\
9.32 \\
9.43 \\
9.54\end{array}$ & $\begin{array}{l}9.10 \\
9.21 \\
9.33 \\
9.44 \\
9.55\end{array}$ & $\begin{array}{l}9.11 \\
9.23 \\
9.34 \\
9.45 \\
9.56\end{array}$ & $\begin{array}{l}9.12 \\
9.24 \\
9.35 \\
9.46 \\
9.57\end{array}$ & $\begin{array}{l}9.14 \\
9.25 \\
9.36 \\
9.47 \\
9.58\end{array}$ & $\begin{array}{l}9.15 \\
9.26 \\
9.37 \\
9.48 \\
9.59\end{array}$ & $\begin{array}{l}9.16 \\
9.27 \\
9.38 \\
9.49 \\
9.61\end{array}$ & 9.6 \\
\hline $\begin{array}{l}0.86 \\
0.87 \\
0.88 \\
0.89\end{array}$ & $\begin{array}{r}9.63 \\
9.74 \\
9.85 \\
9.96 \\
10.08\end{array}$ & $\begin{array}{r}9.64 \\
9.75 \\
9.86 \\
9.98 \\
10.09\end{array}$ & $\begin{array}{r}9.65 \\
9.76 \\
9.87 \\
9.99 \\
10.10\end{array}$ & $\begin{array}{r}9.66 \\
9.77 \\
9.89 \\
10.00 \\
10.11\end{array}$ & $\begin{array}{r}9.67 \\
9.79 \\
9.90 \\
10.01 \\
10.12\end{array}$ & $\begin{array}{r}9.68 \\
9.80 \\
9.91 \\
10.02 \\
10.13\end{array}$ & $\begin{array}{r}9.70 \\
9.81 \\
9.92 \\
10.03 \\
10.14\end{array}$ & $\begin{array}{r}9.71 \\
9.82 \\
9.93 \\
10.04 \\
10.15\end{array}$ & $\begin{array}{r}9.72 \\
9.83 \\
9.94 \\
10.05 \\
10.17\end{array}$ & $\begin{array}{r}9.7 \\
9.8 \\
9.9 \\
10.0 \\
10.1\end{array}$ \\
\hline
\end{tabular}


TABLE 32 (Cont'd)

\section{Oxygen}

Conversion from milligram-atoms per liter to milliliters per liter (I milligram-atom per liter of $\mathrm{O}_{2}=11.196$ milliliters per liter of $\mathrm{O}_{2}$ )

Milligram-

atoms/liter

of $\mathrm{O}_{2}$ $\begin{array}{llllllllll}.000 & .001 & .002 & .003 & .004 & .005 & .006 & .007 & .008 & .009\end{array}$

0.91

0.92

0.93

0.94

0.95

0.96

0.97

0.98

0.99

1.00

1.01

1.02

1.03

1.04

1.05

1.06

1.07

1.08

1.09

1.10

1.11

1.12

1.13

1.14

1.15

1.16

1.17

1.18

1.19

1.20 $\begin{array}{llll}10.19 & 10.20 & 10.21 & 10.22\end{array}$ $\begin{array}{llll}10.30 & 10.31 & 10.32 & 10.33\end{array}$ $\begin{array}{llll}10.41 & 10.42 & 10.43 & 10.45\end{array}$ $\begin{array}{lll}10.52 & 10.54 & 10.55\end{array}$ $\begin{array}{lll}10.64 & 10.65 \quad 10.66\end{array}$ 10.56 10.67

10.57

$10.23 \quad 10.24$

10.26

10.27

$\begin{array}{lll}10.37 & 10.38\end{array}$

10.28

10.39

10.29

10.35

10.36

10.48

10.49

10.50

10.40

10.58

10.59

10.60

10.61

10.51

10.70

10.71

10.73

10.74

$10.75 \quad 10.76$

10.77

10.78

$10.79 \quad 10.80$

10.82

10.83

10.84

10.94

10.95

11.06

11.04

11.05

11.17

11.15

11.16

11.27

11.29

11.38

11.39

11.40

11.51

11.49

11.50

11.60

11.61

11.62

11.73

11.7111 .72

11.85

11.69

11.70

11.82

11.83

11.93

12.05

11.95

11.96

12.06

12.07

12.16

12.17

12.18

12.29

12.41

12.38

12.39

12.36

12.37

12.47

12.48

12.4

12.51

12.52

12.63

12.74

12.70

12.71

12.72

12.62

12.85

12.84

12.96

10.85

10.96

11.07

11.18

12.76

12.77

$12.79 \quad 12.80$

12.81

12.82

12.83

12.95

12.96

$12.99 \quad 13.00$

$13.01 \quad 13.02$

13.03

13.04

13.05

13.07

13.08

13.17

13.18

13.19

$13 \cdot 30$

13.29

13.32

13.33

13.24

13.26

13.27

13.28

13.39

13.40

13.51
13.41

13.52
11.30

11.41

11.52

11.63

11.74

11.86

$\begin{array}{lll}13.44 & 13.45 & 13.46\end{array}$
13.48
13.50
11.97

12.08

12.19

12.30

12.42

12.53

12.64

12.75

12.86

12.98

13.09

13.20

13.31

13.42

13.54 
TABLE 32 (Cont'd)

\section{Oxygen}

Conversion from milligram-atoms per liter to milliliters per liter

( 1 milligram-atom per liter of $\mathrm{O}_{2}=11.196$ milliliters per liter of $\mathrm{O}_{2}$ )

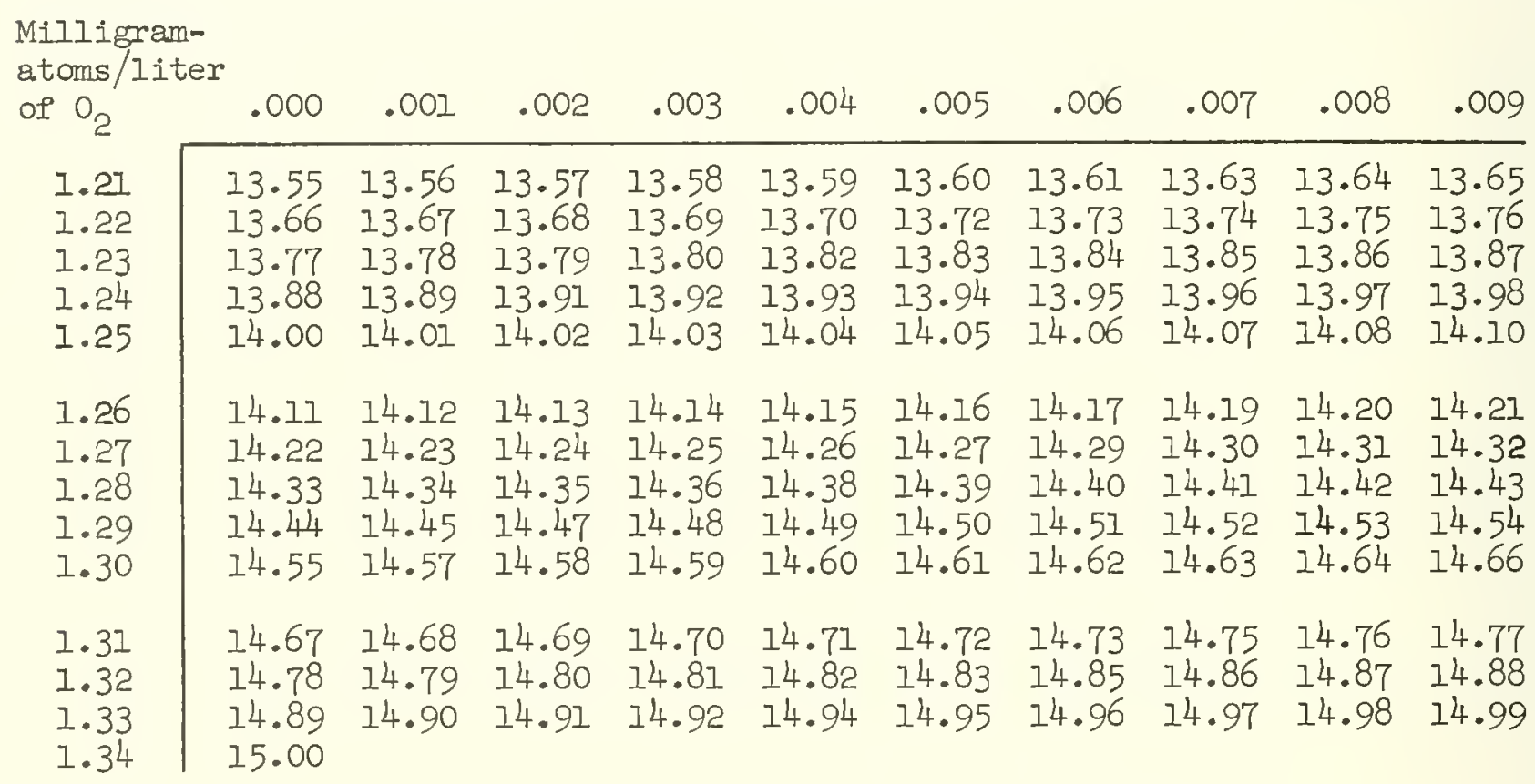


TABLE 33

Phosphorus

Conversion from micrograms per liter of inorganic $P$ to microgram-atoms per liter of $P$

( 1 ug of $\mathrm{P}=0.032285 \mu \mathrm{g}-\mathrm{at}$ of $\mathrm{P}$ )

\section{Micrograms}

per

Liter of

inorganic $\mathrm{P}$

0.0

0.1

0.20 .3

0.4

0.5

0.6

0.7

0.8

0.9

00

$0.00 \quad 0.00$

0.01

0.01

0.01

0.02

0.02

$0.02 \quad 0.03 \quad 0.03$

\section{Micrograms per}

Liter of in-

organic $P$

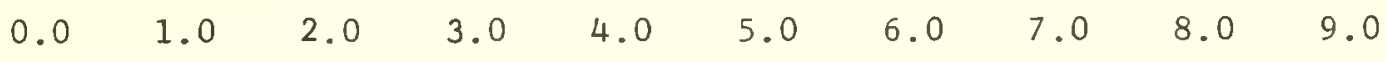

00

$0.00 \quad 0.03 \quad 0.06$

0.10

0.13

0.16

0.19

0.23

0.26

0.29

10

0.32

0.36

0.39

0.420 .45

0.48

0.52

0.55

0.58

0.61

20

0.65

0.68

0.71

0.74

0.77

0.81

0.84

0.87

0.90

0.94

40

0.97

1.03

1.07

1.10

1.13

1.16

1.19

1.23

1.26

50

1.6

1.32

1.36

1.39
1.71

1.42

1.45

1.49

1.52

1.55

1.58

60

$\begin{array}{lll}1.94 & 1.97 & 2.00\end{array}$

2.03

2.07

1.78

1.81

1.84

1.87

1.90

70

80

2.26

2.29

2.32

2.36

2.39

2.10

2.13

2.16

2.20

2.23

$2.58 \quad 2.62$

2.65

2.68

2.71

2.42

2.45

2.49

2. 52

2. 55

$2.91 \quad 2.94$

2.97

3. 00

3.03

2.74

2.78

2.81

2.84

2. 87

3.23

3.26

3.29

3.33

3.36

3.39

3.10

3.13

3.16

3. 20

110

3.55
3.87

$\begin{array}{ll}3.58 & 3.62 \\ 3.91 & 3.94\end{array}$

3.65
3.97

3.68
4.00

3.71

3.75

3.45

3.49

3.52

120

3.87

4.04

4.07

3.78

3.81

3.84

4.10

4.13

4.16 
TABLE 34

Phosphate

Conversion from micrograms per liter of $\mathrm{PO}_{4}$ to microgram-atoms per liter of $\mathrm{PO}_{4}-\mathrm{P}$

(1 ug of $\mathrm{PO}_{4}=0.010529$ ug-at of $\mathrm{PO}_{4}-\mathrm{P}$ )

\section{Micrograms}

per Liter

of $\mathrm{PO}_{4}$

$0.0 \quad 0.1$

0.2

$0.3 \quad 0.4$

0.5

0.6

0.7

0.8

0.9

00

$\begin{array}{lllll}0.00 & 0.00 & 0.00 & 0.00 & 0.00\end{array}$

0.01

0.01

0.01

0.01

0.01

Micrograms

per Liter

of $\mathrm{PO}_{4}$

$\begin{array}{lllll}0.0 & 1.0 & 2.0 & 3.0 & 4.0\end{array}$

5.0

6.0

7.0

8.0

9.0

\begin{tabular}{|c|c|c|c|c|c|c|c|c|c|c|}
\hline 00 & 0.00 & 0.01 & 0.02 & 0.03 & 0.04 & 0.05 & 0.06 & 0.07 & 0.08 & 0.09 \\
\hline 10 & 0.11 & 0.12 & 0.13 & 0.14 & 0.15 & 0.16 & 0.17 & 0.18 & 0.19 & 0.20 \\
\hline 20 & 0.21 & 0.22 & 0.23 & 0.24 & 0.25 & 0.26 & 0.27 & 0.28 & 0.29 & 0.31 \\
\hline 30 & 0.32 & 0.33 & 0.34 & 0.35 & 0.36 & 0.37 & 0.38 & 0.39 & 0.40 & 0.41 \\
\hline 40 & 0.42 & 0.43 & 0.44 & 0.45 & 0.46 & 0.47 & 0.48 & 0.49 & 0.51 & 0.52 \\
\hline 50 & 0.53 & 0.54 & 0.55 & 0.56 & 0.57 & 0.58 & 0.59 & 0.60 & 0.61 & 0.62 \\
\hline 60 & 0.63 & 0.64 & 0.65 & 0.66 & 0.67 & 0.68 & 0.69 & 0.71 & 0.72 & 0.73 \\
\hline 70 & 0.74 & 0.75 & 0.76 & 0.77 & 0.78 & 0.79 & 0.80 & 0.81 & 0.82 & 0.83 \\
\hline 80 & 0.84 & 0.85 & 0.86 & 0.87 & 0.88 & 0.89 & 0.91 & 0.92 & 0.93 & 0.94 \\
\hline 90 & 0.95 & 0.96 & 0.97 & 0.98 & 0.99 & 1.00 & 1.01 & 1.02 & 1.03 & 1.04 \\
\hline 100 & 1.05 & 1.06 & 1.07 & 1.08 & 1.10 & 1.11 & 1.12 & 1.13 & 1.14 & 1.15 \\
\hline 110 & 1.16 & 1.17 & 1.18 & 1.19 & 1.20 & 1.21 & 1.22 & 1.23 & 1.24 & 1.25 \\
\hline 120 & 1.26 & 1.27 & 1.28 & 1.30 & 1.31 & 1.32 & 1.33 & 1.34 & 1.35 & 1.36 \\
\hline 130 & 1.37 & 1.38 & 1.39 & 1.40 & 1.41 & 1.42 & 1.43 & 1.44 & 1.45 & 1.46 \\
\hline 140 & 1.47 & 1.48 & 1.50 & 1.51 & 1.52 & 1.53 & 1.54 & 1.55 & 1.56 & 1.57 \\
\hline 150 & 1.58 & 1.59 & 1.60 & 1.61 & 1.62 & 1.63 & 1.64 & 1.65 & 1.66 & 1.67 \\
\hline 160 & 1.68 & 1.70 & 1.71 & 1.72 & 1.73 & 1.74 & 1.75 & 1.76 & 1.77 & 1.78 \\
\hline 170 & 1.79 & 1.80 & 1.81 & 1.82 & 1.83 & 1.84 & 1.85 & 1.86 & 1.87 & 1.88 \\
\hline 180 & 1.90 & 1.91 & 1.92 & 1.93 & 1.94 & 1.95 & 1.96 & 1.97 & 1.98 & 1.99 \\
\hline 190 & 2.00 & 2.01 & 2.02 & 2.03 & 2.04 & 2.05 & 2.06 & 2.07 & 2.08 & 2.10 \\
\hline 200 & 2.11 & 2.12 & 2.13 & 2.14 & 2.15 & 2.16 & 2.17 & 2.18 & 2.19 & 2.20 \\
\hline 210 & 2.21 & 2.22 & 2.23 & 2.24 & 2.25 & 2.26 & 2.27 & 2.28 & 2.30 & 2.31 \\
\hline 220 & 2.32 & 2.33 & 2.34 & 2.35 & 2.36 & 2.37 & 2.38 & 2.39 & 2.40 & 2.41 \\
\hline 230 & 2.42 & 2.43 & 2.44 & 2.45 & 2.46 & 2.47 & 2.48 & 2.50 & 2.51 & 2.52 \\
\hline 240 & 2.53 & 2.54 & 2.55 & 2.56 & 2.57 & 2.58 & 2.59 & 2.60 & 2.61 & 2.62 \\
\hline 250 & 2.63 & 2.64 & 2.65 & 2.66 & 2.67 & 2.68 & 2.70 & 2.71 & 2.72 & 2.73 \\
\hline
\end{tabular}




\section{TABLE 34 ( Cont $^{1} \mathrm{~d}$ )}

Phosphate

Conversion from micrograms per liter of $\mathrm{PO}_{4}$ to microgram-atoms per liter of $\mathrm{PO}_{4}-\mathrm{P}$

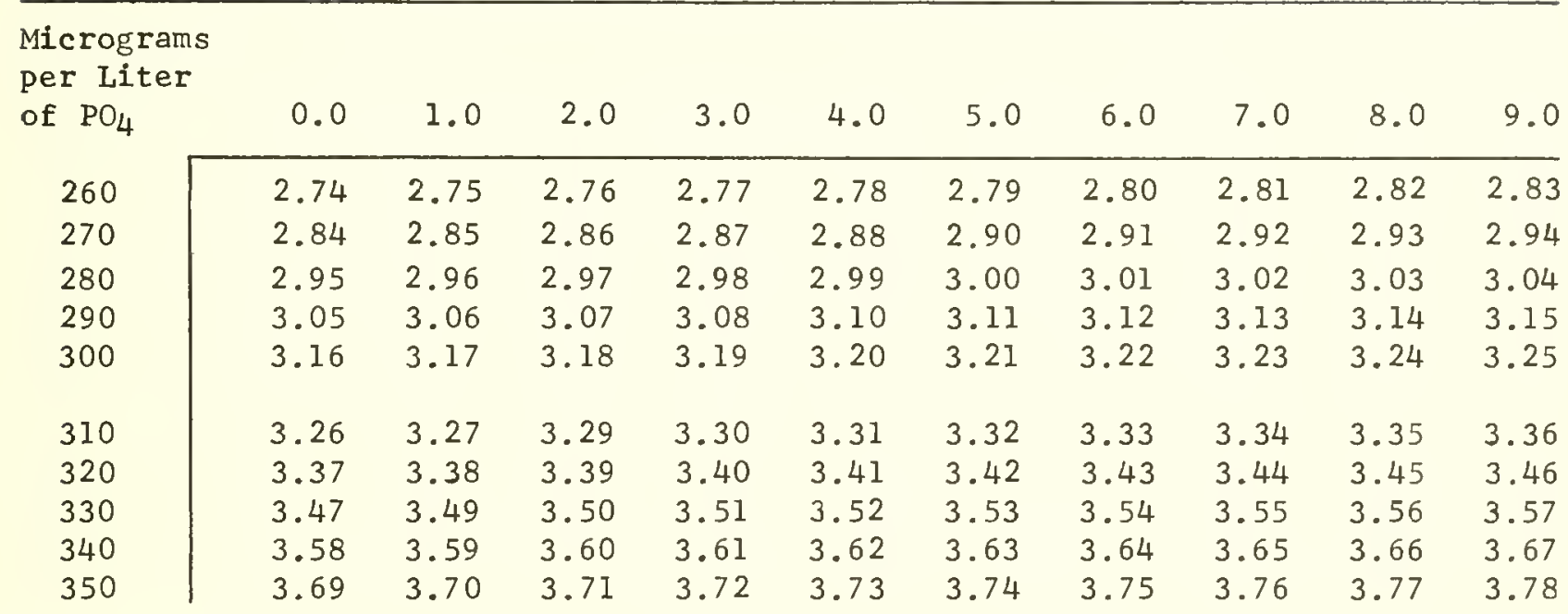


Phosphorus Pentoxide

Conversion from micrograms per liter of $\mathrm{P}_{2} \mathrm{O}_{5}$ to microgram-atoms per liter of $P$ (1 ug of $\mathrm{P}_{2} \mathrm{O}_{5}=0.014090 \mu g$-atom of $\mathrm{P}$ )

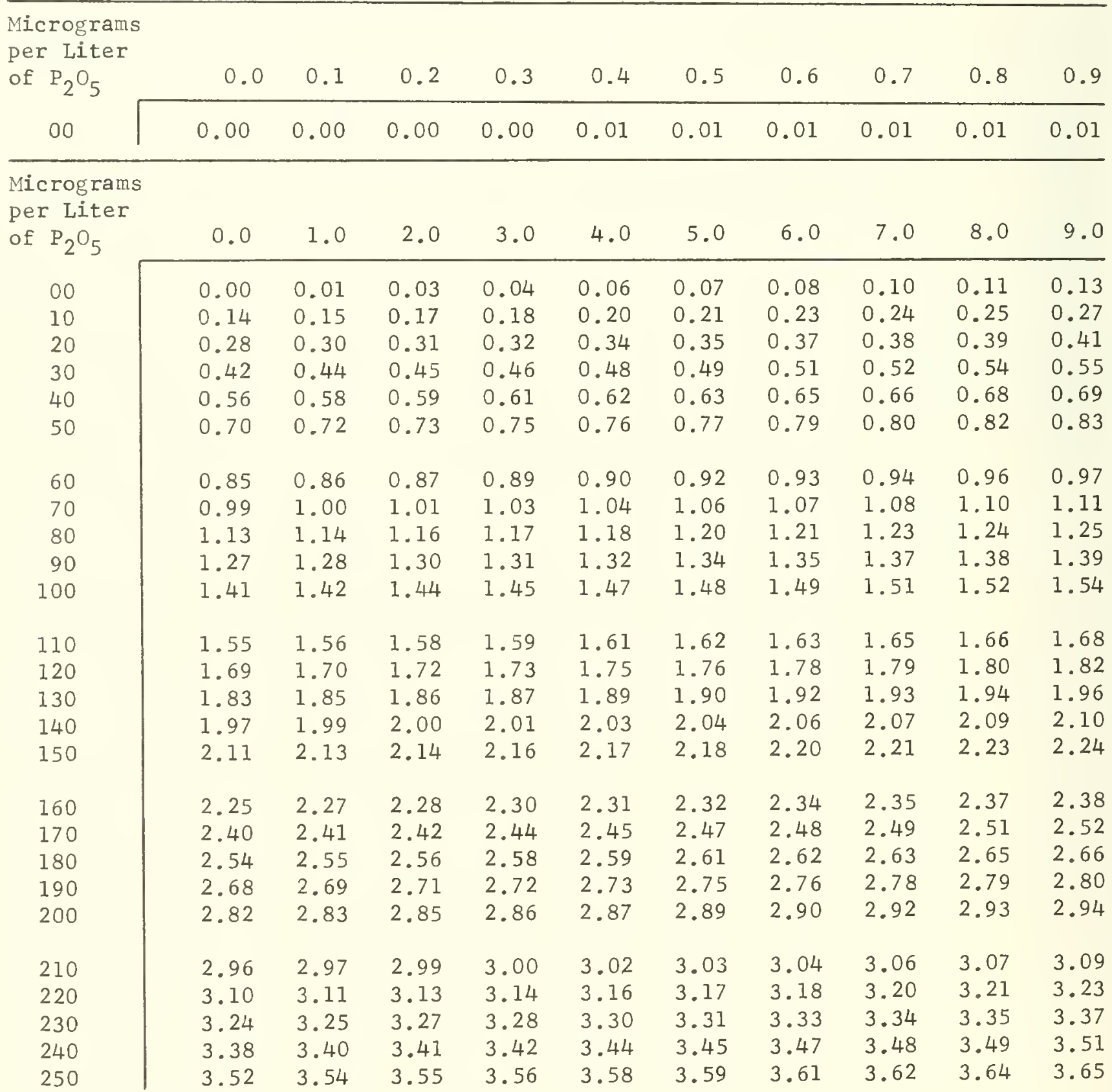

Note: For values greater than 259, the conversion is to be obtained by addition. 
TABLE 36

Nitrite

Conversion from micrograms per liter of $\mathrm{NO}_{2}$ to microgram-atoms per liter of $\mathrm{NO}_{2}-\mathrm{N}$ (1 $\mathrm{\mu g}$ of $\mathrm{NO}_{2}=0.0217365^{2} \mathrm{ug}$ - at of $\mathrm{NO}_{2}-\mathrm{N}$ )

Micrograms

per Liter

\begin{tabular}{|c|c|c|c|c|c|c|c|c|c|c|}
\hline of $\mathrm{NO}_{2}$ & 0.0 & 1.0 & 2.0 & 3.0 & 4.0 & 5.0 & 6.0 & 7.0 & 8.0 & 9.0 \\
\hline 00 & 0.00 & 0.02 & 0.04 & 0.07 & 0.09 & 0.11 & 0.13 & 0.15 & 0.17 & 0.20 \\
\hline 10 & 0.22 & 0.24 & 0.26 & 0.28 & 0.30 & 0.33 & 0.35 & 0.37 & 0.39 & 0.41 \\
\hline 20 & 0.43 & 0.46 & 0.48 & 0.50 & 0.52 & 0.54 & 0.57 & 0.59 & 0.61 & 0.63 \\
\hline 30 & 0.65 & 0.67 & 0.70 & 0.72 & 0.74 & 0.76 & 0.78 & 0.80 & 0.83 & 0.85 \\
\hline 40 & 0.87 & 0.89 & 0.91 & 0.93 & 0.96 & 0.98 & 1.00 & 1.02 & 1.04 & 1.07 \\
\hline 50 & 1.09 & 1.11 & 1.13 & 1.15 & 1.17 & 1.20 & 1.22 & 1.24 & 1.26 & 1.28 \\
\hline 60 & 1.30 & 1.33 & 1.35 & 1.37 & 1.39 & 1.41 & 1.43 & 1.46 & 1.48 & 1.50 \\
\hline 70 & 1.52 & 1.54 & 1.57 & 1.59 & 1.61 & 1.63 & 1.65 & 1.67 & 1.70 & 1.72 \\
\hline 80 & 1.74 & 1.76 & 1.78 & 1.80 & 1.83 & 1.85 & 1.87 & 1.89 & 1.91 & 1.93 \\
\hline 90 & 1.96 & 1.98 & 2.00 & 2.02 & 2.04 & 2.06 & 2.09 & 2.11 & 2.13 & 2.15 \\
\hline 100 & 2.17 & 2.20 & 2.22 & 2.24 & 2.26 & 2.28 & 2.30 & 2.33 & 2.35 & 2.37 \\
\hline 110 & 2.39 & 2.41 & 2.43 & 2.46 & 2.48 & 2.50 & 2.52 & 2.54 & 2.56 & 2.59 \\
\hline 120 & 2.61 & 2.63 & 2.65 & 2.67 & 2.70 & 2.72 & 2.74 & 2.76 & 2.78 & 2.80 \\
\hline 130 & 2.83 & 2.85 & 2.87 & 2.89 & 2.91 & 2.93 & 2.96 & 2.98 & 3.00 & 3.02 \\
\hline 140 & 3.04 & 3.06 & 3.09 & 3.11 & 3.13 & 3.15 & 3.17 & 3.20 & 3.22 & 3.24 \\
\hline 150 & 3.26 & 3.28 & 3.30 & 3.33 & 3.35 & 3.37 & 3.39 & 3.41 & 3.43 & 3.46 \\
\hline 160 & 3.48 & 3.50 & 3.52 & 3.54 & 3.56 & 3.59 & 3.61 & 3.63 & 3.65 & 3.67 \\
\hline 170 & 3.70 & 3.72 & 3.74 & 3.76 & 3.78 & 3.80 & 3.83 & 3.85 & 3.87 & 3.89 \\
\hline 180 & 3.91 & 3.93 & 3.96 & 3.98 & 4.00 & 4.02 & 4.04 & 4.06 & 4.09 & 4.11 \\
\hline 190 & 4.13 & 4.15 & 4.17 & 4.20 & 4.22 & 4.24 & 4.26 & 4.28 & 4.30 & 4.33 \\
\hline ח & 4.35 & 4.37 & 4.39 & 4.41 & 4.43 & 4.46 & 4.48 & 4.50 & 4.52 & 4.54 \\
\hline
\end{tabular}


TABLE 37

Nitrate

Conversion from micrograms per liter of $\mathrm{NO}_{3}$ to microgram-atoms per liter of $\mathrm{NO}_{3}-\mathrm{N}$

Micrograms per

liter of $\mathrm{NO}_{3}$

01

$02-03$

$03 \quad 04$

\begin{tabular}{|c|c|c|c|c|c|c|c|c|c|c|}
\hline 00 & 00.0 & 00.0 & 00.0 & 00.0 & 00.1 & 00.1 & 00.1 & 00.1 & 00.1 & 00.1 \\
\hline 10 & 00.2 & 00.2 & 00.2 & 00.2 & 00.2 & 00.2 & 00.3 & 00.3 & 00.3 & 00.3 \\
\hline 20 & 00.3 & 00.3 & 00.4 & 00.4 & 00.4 & 00.4 & 00.4 & 00.4 & 00.5 & 00.5 \\
\hline 30 & 00.5 & 00.5 & 00.5 & 00.5 & 00.5 & 00.6 & 00.6 & 00.6 & 00.6 & 00.6 \\
\hline 40 & 00.6 & 00.7 & 00.7 & 00.7 & 00.7 & 00.7 & 00.7 & 00.8 & 00.8 & 00.8 \\
\hline 50 & 00.8 & 00.8 & 00.8 & 00.9 & 00.9 & 00.9 & 00.9 & 00.9 & 00.9 & 01.0 \\
\hline 60 & 01.0 & 01.0 & 01.0 & 01.0 & 01.0 & 01.0 & 01.1 & 01.1 & 01.1 & 01.1 \\
\hline 70 & 01.1 & 01.1 & 01.2 & 01.2 & 01.2 & 01.2 & 01.2 & 01.2 & 01.3 & 01.3 \\
\hline 80 & 01.3 & 01.3 & 01.3 & 01.3 & 01.4 & 01.4 & 01.4 & 01.4 & 01.4 & 01.4 \\
\hline 90 & 01.5 & 01.5 & 01.5 & 01.5 & 01.5 & 01.5 & 01.5 & 01.6 & 01.6 & 01.6 \\
\hline
\end{tabular}

Micrograms per

liter of $\mathrm{NO}_{3}$

00

100

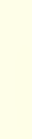

300

400

500

$\begin{array}{llllllllll}01.6 & 01.8 & 01.9 & 02.1 & 02.3 & 02.4 & 02.6 & 02.7 & 02.9 & 03.1 \\ 03.2 & 03.4 & 03.5 & 03.7 & 03.9 & 04.0 & 04.2 & 04.4 & 04.5 & 04.7 \\ 04.8 & 05.0 & 05.2 & 05.3 & 05.5 & 05.6 & 05.8 & 06.0 & 06.1 & 06.3 \\ 06.5 & 06.6 & 06.8 & 06.9 & 07.1 & 07.3 & 07.4 & 07.6 & 07.7 & 07.9 \\ 08.1 & 08.2 & 08.4 & 08.5 & 08.7 & 08.9 & 09.0 & 09.2 & 09.4 & 09.5 \\ 09.7 & 09.8 & 10.0 & 10.2 & 10.3 & 10.5 & 10.6 & 10.8 & 11.0 & 11.1 \\ 11.3 & 11.5 & 11.6 & 11.8 & 11.9 & 12.1 & 12.3 & 12.4 & 12.6 & 12.7 \\ 12.9 & 13.1 & 13.2 & 13.4 & 13.5 & 13.7 & 13.9 & 14.0 & 14.2 & 14.4 \\ 14.5 & 14.7 & 14.8 & 15.0 & 15.2 & 15.3 & 15.5 & 15.6 & 15.8 & 16.0 \\ 16.1 & 16.3 & 16.5 & 16.6 & 16.8 & 16.9 & 17.1 & 17.3 & 17.4 & 17.6 \\ 17.7 & 17.9 & 18.1 & 18.2 & 18.4 & 18.5 & 18.7 & 18.9 & 19.0 & 19.2 \\ 19.4 & 19.5 & 19.7 & 19.8 & 20.0 & 20.2 & 20.3 & 20.5 & 20.6 & 20.8 \\ 21.0 & 21.1 & 21.3 & 21.4 & 21.6 & 21.8 & 21.9 & 22.1 & 22.3 & 22.4 \\ 22.6 & 22.7 & 22.9 & 23.1 & 23.2 & 23.4 & 23.5 & 23.7 & 23.9 & 24.0 \\ 24.2 & 24.4 & 24.5 & 24.7 & 24.8 & 25.0 & 25.2 & 25.3 & 25.5 & 25.6 \\ 25.8 & 26.0 & 26.1 & 26.3 & 26.4 & 26.6 & 26.8 & 26.9 & 27.1 & 27.3 \\ 27.4 & 27.6 & 27.7 & 27.9 & 28.1 & 28.2 & 28.4 & 28.5 & 28.7 & 28.9 \\ 29.0 & 29.2 & 29.4 & 29.5 & 29.7 & 29.8 & 30.0 & 30.2 & 30.3 & 30.5 \\ 30.6 & 30.8 & 31.0 & 31.1 & 31.3 & 31.4 & 31.6 & 31.8 & 31.9 & 32.1 \\ 32.3 & 32.4 & 32.6 & 32.7 & 32.9 & 33.1 & 33.2 & 33.4 & 33.5 & 33.7 \\ & & & & & & & & & \end{array}$


TABLE 37 (Cont' $\mathrm{d}$ )

Nitrate

Conversion from micrograms per liter of $\mathrm{NO}_{3}$ to microgram-atoms per liter of $\mathrm{NO}_{3}-\mathrm{N}$

\begin{tabular}{|c|c|c|c|c|c|c|c|c|c|c|}
\hline $\begin{array}{l}\text { Micrograms } \\
\text { liter of } \mathrm{NO}_{3}\end{array}$ & 00 & 10 & 20 & 30 & 40 & 50 & 60 & 70 & 80 & 90 \\
\hline 2100 & 33.9 & 34.0 & 34.2 & 34.4 & 34.5 & 34.7 & 34.8 & 35.0 & 35.2 & 35.3 \\
\hline 2200 & 35.5 & 35.6 & 35.8 & 36.0 & 36.1 & 36.3 & 36.4 & 36.6 & 36.8 & 36.9 \\
\hline 2300 & 37.1 & 37.3 & 37.4 & 37.6 & 37.7 & 37.9 & 38.1 & 38.2 & 38.4 & 38.5 \\
\hline 2400 & 38.7 & 38.9 & 39.0 & 39.2 & 39.4 & 39.5 & 39.7 & 39.8 & 40.0 & 40.2 \\
\hline 2500 & 40.3 & 40.5 & 40.6 & 40.8 & 41.0 & 41.1 & 41.3 & 41.4 & 41.6 & 41.8 \\
\hline 2600 & 41.9 & 42.1 & 42.3 & 42.4 & 42.6 & 42.7 & 42.9 & 43.1 & 43.2 & 43.4 \\
\hline 2700 & 43.5 & 43.7 & 43.9 & 44.0 & 44.2 & 44.4 & 44.5 & 44.7 & 44.8 & 45.0 \\
\hline 2800 & 45.2 & 45.3 & 45.5 & 45.6 & 45.8 & 46.0 & 46.1 & 46.3 & 46.4 & 46.6 \\
\hline 2900 & 46.8 & 46.9 & 47.1 & 47.3 & 47.4 & 47.6 & 47.7 & 47.9 & 48.1 & 48.2 \\
\hline 3000 & 48.4 & 48.5 & 48.7 & 48.9 & 49.0 & 49.2 & 49.4 & 49.5 & 49.7 & 49.8 \\
\hline
\end{tabular}

NOTE: Conversion of values not given directly in the tables are derived by addition. 
Silicon

Conversion from micrograms per liter of Si to microgram-atoms per liter of Si ( $1 \mathrm{\mu g}$ of $\mathrm{Si}=0.0356049 \mu \mathrm{g}$-atom Si)

\begin{tabular}{|c|c|c|c|c|c|c|c|c|c|c|}
\hline \multicolumn{11}{|l|}{$\begin{array}{c}\text { Micrograms } \\
\text { per }\end{array}$} \\
\hline 000 & 000 & 000 & 001 & 001 & 001 & 002 & 002 & 002 & 003 & 003 \\
\hline 100 & 004 & 004 & 004 & 005 & 005 & 005 & 006 & 006 & 006 & 007 \\
\hline 200 & 007 & 007 & 008 & 008 & 009 & 009 & 009 & 010 & 010 & 010 \\
\hline 300 & 011 & 011 & 011 & 012 & 012 & 012 & 013 & 013 & 014 & 014 \\
\hline 400 & 014 & 015 & 015 & 015 & 016 & 016 & 016 & 017 & 017 & 017 \\
\hline 500 & 018 & 018 & 019 & 019 & 019 & 020 & 020 & 020 & 021 & 021 \\
\hline 600 & 021 & 022 & 022 & 022 & 023 & 023 & 023 & 024 & 024 & 025 \\
\hline 700 & 025 & 025 & 026 & 026 & 026 & 027 & 027 & 027 & 028 & 028 \\
\hline 800 & 028 & 029 & 029 & 030 & 030 & 030 & 031 & 031 & 031 & 032 \\
\hline 900 & 032 & 032 & 033 & 033 & 033 & 034 & 034 & 035 & 035 & 035 \\
\hline \multicolumn{11}{|l|}{$\begin{array}{c}\text { Micrograms } \\
\text { per }\end{array}$} \\
\hline Liter of $\mathrm{Si}$ & 000 & 100 & 200 & 300 & 400 & 500 & 600 & 700 & 800 & 900 \\
\hline 1000 & 036 & 039 & 043 & 046 & 050 & 053 & 057 & 061 & 064 & 068 \\
\hline 2000 & 071 & 075 & 078 & 082 & 085 & 089 & 093 & 096 & 100 & 103 \\
\hline 3000 & 107 & 110 & 114 & 117 & 121 & 125 & 128 & 132 & 135 & 139 \\
\hline 4000 & 142 & 146 & 150 & 153 & 157 & 160 & 164 & 1.67 & 171 & 174 \\
\hline 5000 & 178 & 182 & 185 & 189 & 192 & 196 & 199 & 203 & 207 & 210 \\
\hline 6000 & 214 & 217 & 221 & 224 & 228 & 231 & 235 & 239 & 242 & 246 \\
\hline 7000 & 249 & 253 & 256 & 260 & 263 & 267 & 271 & 274 & 278 & 281 \\
\hline 8000 & 285 & 288 & 292 & 296 & 299 & 303 & 306 & 310 & 313 & 317 \\
\hline
\end{tabular}

EXAMPLE I:

Assume an initial value of 4200. Since this value lies within the range 1000 - 8900, use lower portion of above table. Enter left hand column at 4000 , proceed horizontally to the right to column headed 200 , and read 150 .

EXAMPLE II:

Assume an initial value of 4180 . Since this value is not recorded explicitly in the table, the conversion can be made by one of two methods: 
TABLE 38 (Cont'd)

Silicon

(1) Interpolation between 4100 and 4200 to nearest whole number, 149 :

or

(2) Since $4180=4100+80$, find 146 corresponding to 4100 and 003 corresponding to 80 . Add 146 and $\underline{003}$ to get 149 . 
Silicon Dioxide

Conversion from micrograms per liter of $\mathrm{SiO}_{2}$ to microgram-atoms per liter of $\mathrm{SiO}_{2}-\mathrm{SI}$ (1 $\mu g$ of $\mathrm{SiO}_{2}=0.016643^{2} \mu g$-a.tom of $\mathrm{SI}$ )

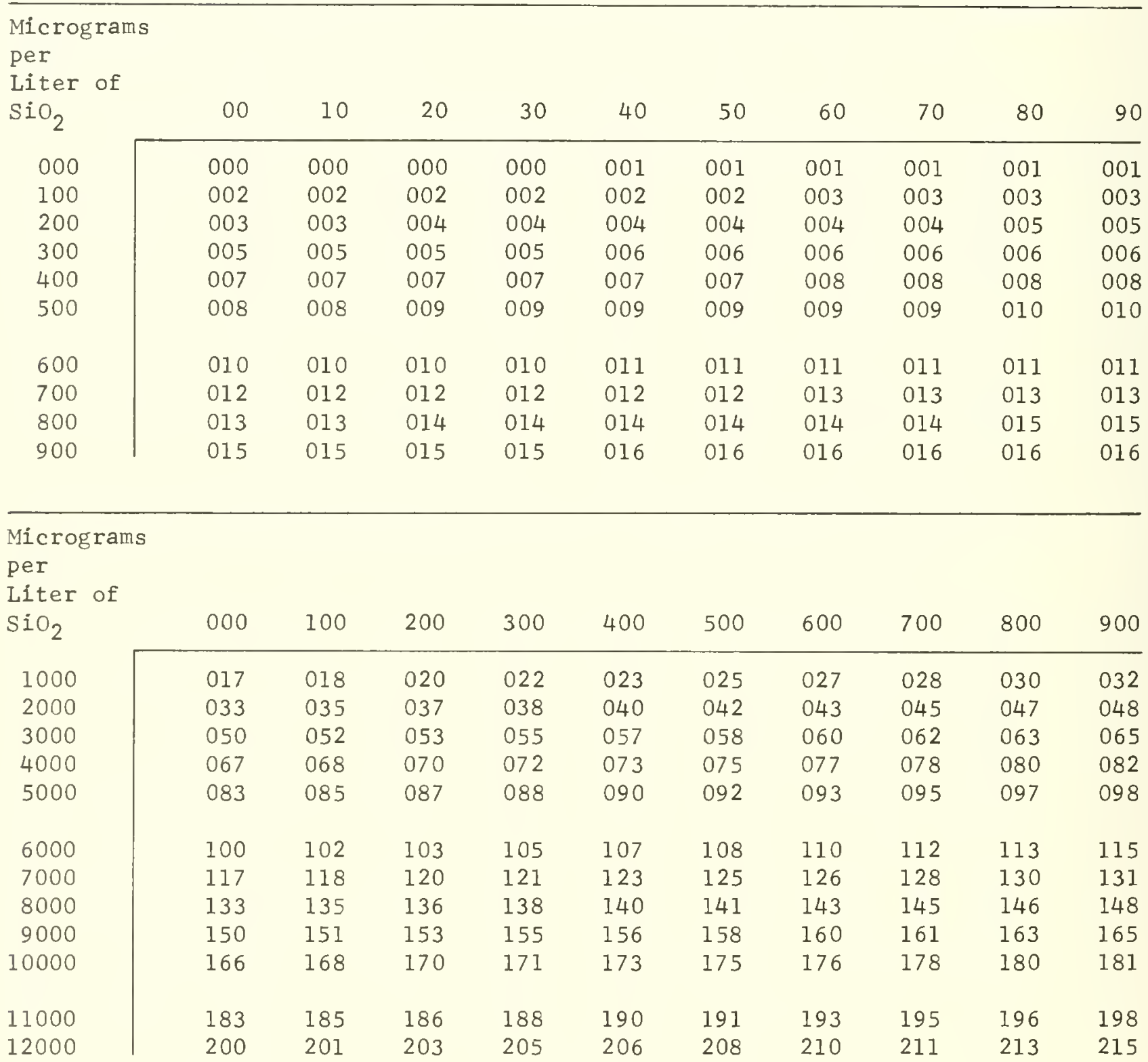


Silicate

Conversion from milligrams per liter of $\mathrm{SiO}_{3}$ to microgram-atoms per liter of $\mathrm{SiO}_{3}$-Si ( 1 milligram of $\mathrm{SiO}_{3}=13.1433$ microgram-atoms of $\mathrm{SiO}_{3}-\mathrm{Si}$ )

\begin{tabular}{|c|c|c|c|c|c|c|c|c|c|c|}
\hline \multicolumn{11}{|l|}{$\begin{array}{l}\text { Milligrams } \\
\text { per Liter }\end{array}$} \\
\hline of $\mathrm{SiO}_{3}$ & 0.0 & 0.1 & 0.2 & 0.3 & 0.4 & 0.5 & 0.6 & 0.7 & 0.8 & 0.9 \\
\hline 00 & 000 & 001 & 003 & 004 & 005 & 007 & 008 & 009 & 011 & 012 \\
\hline 01 & 013 & 014 & 016 & 017 & 018 & 020 & 021 & 022 & 024 & 025 \\
\hline 02 & 026 & 028 & 029 & 030 & 032 & 033 & 034 & 035 & 037 & 038 \\
\hline 03 & 039 & 041 & 042 & 043 & 045 & 046 & 047 & 049 & 050 & 051 \\
\hline 04 & 053 & 054 & 055 & 057 & 058 & 059 & 060 & 062 & 063 & 064 \\
\hline 05 & 066 & 067 & 068 & 070 & 071 & 072 & 074 & 075 & 076 & 078 \\
\hline 06 & 079 & 080 & 081 & 083 & 084 & 085 & 087 & 088 & 089 & 091 \\
\hline 07 & 092 & 093 & 095 & 096 & 097 & 099 & 100 & 101 & 103 & 104 \\
\hline 08 & 105 & 106 & 108 & 109 & 110 & 112 & 113 & 114 & 116 & 117 \\
\hline 09 & 118 & 120 & 121 & 122 & 124 & 125 & 126 & 127 & 129 & 130 \\
\hline 10 & 131 & 133 & 134 & 135 & 137 & 138 & 139 & 141 & 142 & 143 \\
\hline 11 & 145 & 146 & 147 & 149 & 150 & 151 & 152 & 154 & 155 & 156 \\
\hline 12 & 158 & 159 & 160 & 162 & 163 & 164 & 166 & 167 & 168 & 170 \\
\hline 13 & 171 & 172 & 173 & 175 & 176 & 177 & 179 & 180 & 181 & 183 \\
\hline 14 & 184 & 185 & 187 & 188 & 189 & 191 & 192 & 193 & 195 & 196 \\
\hline 15 & 197 & 198 & 200 & 201 & 202 & 204 & 205 & 206 & 208 & 209 \\
\hline 16 & 210 & 212 & 213 & 214 & 216 & 217 & 218 & 219 & 221 & 222 \\
\hline 17 & 223 & 225 & 226 & 227 & 229 & 230 & 231 & 233 & 234 & 235 \\
\hline 18 & 237 & 238 & 239 & 241 & 242 & 243 & 244 & 246 & 247 & 248 \\
\hline 19 & 250 & 251 & 252 & 254 & 255 & 256 & 258 & 259 & 260 & 262 \\
\hline 20 & 263 & 264 & 265 & 267 & 268 & 269 & 271 & 272 & 273 & 275 \\
\hline
\end{tabular}





\section{APPENDIX 1}

MATIOHAL OCEAMOGRAPHIC OATA CEHTER

WLSHAMGIOQ 2S, OC

PHYSICAL AND CHEMICAL DATA FORM

NHO.NODC. $3167 / 1(9.61)$

FOR OCEANOGRAPHIC STATIONS

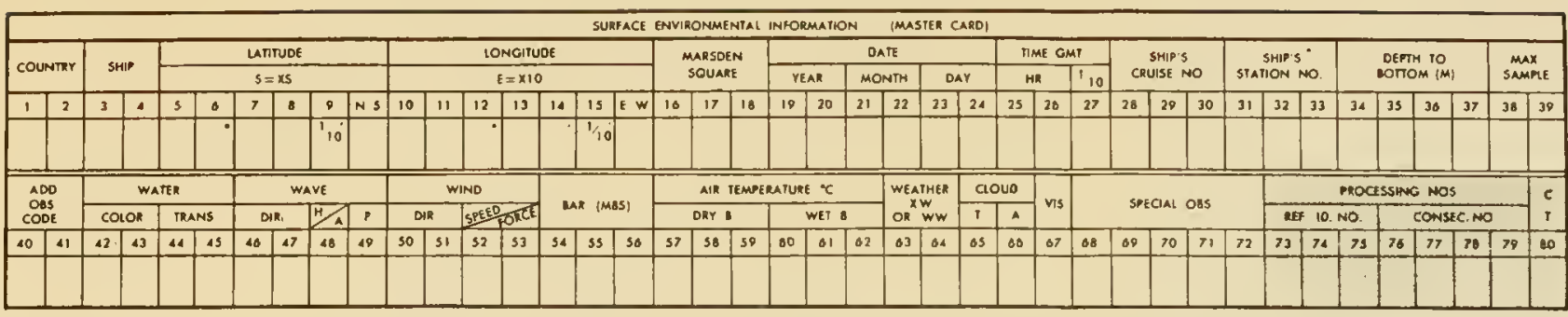

\begin{tabular}{|l|}
\hline COUNTRY \\
\hline INSTIIUTE \\
\hline VESSEL \\
\hline CRUISE OR MONECT NO \\
\hline
\end{tabular}

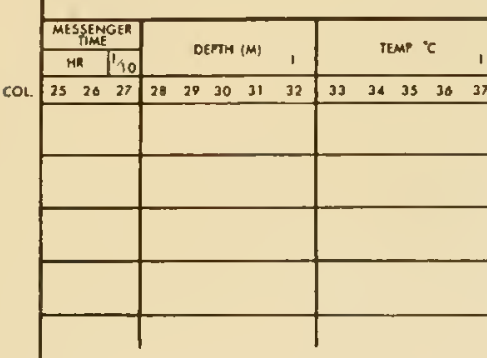

SUGSURFACE OBSERYATIONS-(DETAIL CARDS)

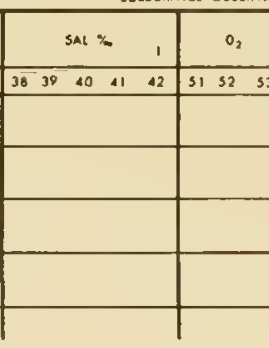

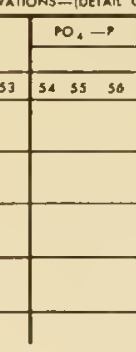

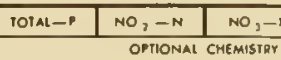

REMarks

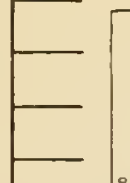

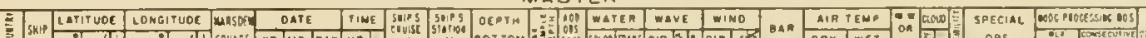

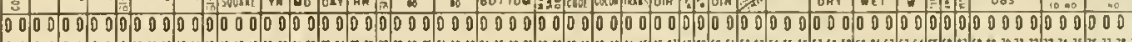

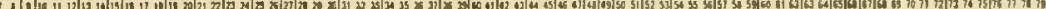

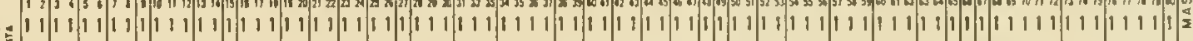

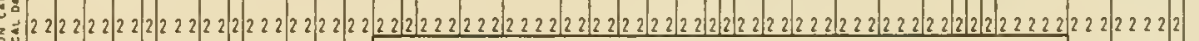

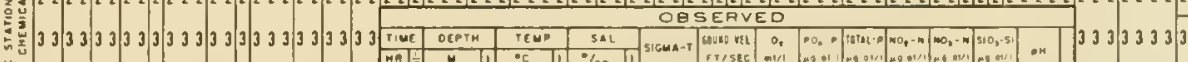

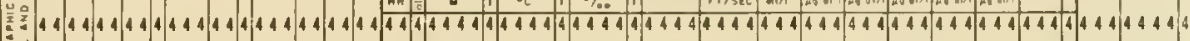

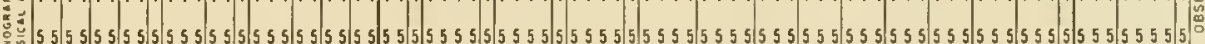

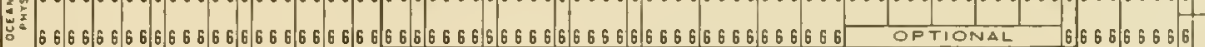

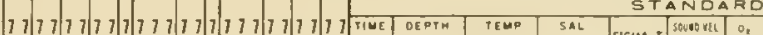

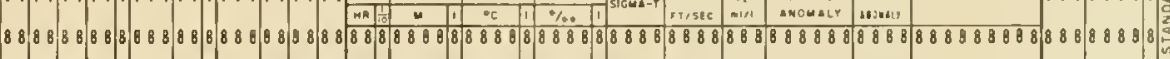
9999999999999999999999999999999999999999999999999999999999999999999999599999999999999999

\section{OCEANOGRAPHIC STATION CARD} PHYSICAL AND CHEMICAL DATA

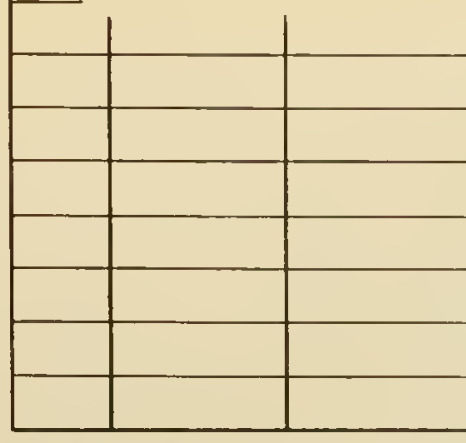

$\mid$

$|+|$

$\mid$

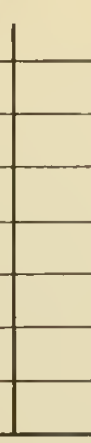

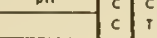

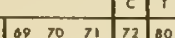

\begin{tabular}{|c|c|}
\hline \\
\hline
\end{tabular}

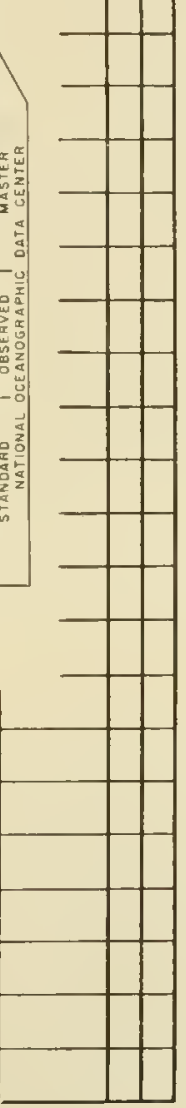

105 



$$
\text { , }
$$

